\title{
Resilient Stochastic Control Strategies in Cyber-Physical Microgrids
}

\author{
Mohammad Reza Khalghani \\ West Virginia University, mk0005@mix.wvu.edu
}

Follow this and additional works at: https://researchrepository.wvu.edu/etd

Part of the Power and Energy Commons

\section{Recommended Citation}

Khalghani, Mohammad Reza, "Resilient Stochastic Control Strategies in Cyber-Physical Microgrids" (2019). Graduate Theses, Dissertations, and Problem Reports. 7483.

https://researchrepository.wvu.edu/etd/7483

This Dissertation is protected by copyright and/or related rights. It has been brought to you by the The Research Repository @ WVU with permission from the rights-holder(s). You are free to use this Dissertation in any way that is permitted by the copyright and related rights legislation that applies to your use. For other uses you must obtain permission from the rights-holder(s) directly, unless additional rights are indicated by a Creative Commons license in the record and/ or on the work itself. This Dissertation has been accepted for inclusion in WVU Graduate Theses, Dissertations, and Problem Reports collection by an authorized administrator of The Research Repository @ WVU.

For more information, please contact researchrepository@mail.wvu.edu. 


\title{
Resilient Stochastic Control Strategies in Cyber-Physical Microgrids
}

\section{Mohammad Reza Khalghani}

\author{
Dissertation submitted to the \\ Benjamin M. Statler College of Engineering and Mineral Resources \\ at West Virginia University \\ in partial fulfillment of the requirements for the degree of
}

\author{
Doctor of Philosophy \\ in \\ Electrical Engineering \\ Jignesh Solanki, Ph.D., Chair \\ Sarika Solanki, Ph.D. \\ Muhammad A . Choudhry, Ph.D. \\ Natalia Schmid, D.Sc. \\ Debangsu Bhattacharyya, Ph.D.
}

Lane Department of Computer Science and Electrical Engineering

Morgantown, West Virginia

2019

Keywords: Microgrid Modeling and Control, Artificial Intelligence Techniques, Resiliency, Electric Vehicle, Stochastic Modeling, Cyber Disruption

Copyright 2019 Mohammad Reza Khalghani 


\section{Abstract \\ Resilient Stochastic Control Strategies in Cyber-Physical Microgrids}

\section{Mohammad Reza Khalghani}

Microgrids can locally fulfill the demand and operate isolated from the main grid to sustain critical services even in case of large-scale outages and cascading power failures. Microgrids are the integration of a large number of distributed energy resources in a decentralized way such that the energy supply reliability and resiliency are enhanced against natural disasters, physical and cyber disruptions. This dissertation focuses on three main challenges regarding technologies emerging in microgrids. 1) According to the fast growing number of Electric Vehicles (EV) deployment, their impacts on microgrids resulted from their uncertain behaviors are new concerns for the microgrid operators. These concerns can be EV charging and discharging schedule and locations of EV parking-lots in the system which are considered and solved using stochastic modeling and optimization algorithms based on Artificial Intelligence (AI) techniques. First, charging and discharging scheduling of EVs is minimized using Particle Swarm Optimization (PSO) Algorithm such that the daily load profile is flattened (peak and off-peak shaving) with considering constraints of State-Of-Charging (SoC). Due to the uncertain nature of daily travelling EVs between residential and administrative areas, chronological stochastic modeling is suggested. Furthermore, a multi-objective optimization based on PSO and fuzzification theory is proposed to find the best location of parking lots for these EV aggregators. Two indices, voltage unbalance and power loss, for locating the EV aggregators are considered. During peak hour, these indices can be more critical for a three-phase distribution system. 2) As the penetration of renewable energies, generally, uncertainties, increase in microgrids, a more dynamic and complex system is emerging that makes frequency control of the islanded microgrid more challenging due to stochastic dyna mic encumbrances. These stochastic encumbrances can create oscillatory frequency response, which eventually leads to astray controls and instability even under primary and traditional secondary controllers. This dissertation develops AI-based and analytical based secondary control for the islanded microgrids to compensate for the frequency deviation in the presence of intermittent energy resources and uncertain load changes. Two types of artificial 
intelligent techniques, Hebb learning controller, and human brain emotional learning controller, are suggested to maintain the balance between load and power sources in the presence of uncertainties. These techniques are model-free methods with self-tuning Proportional-Integral-Derivative (PID) structure. 3) The increased integration of cyber and communication networks into secondary control of microgrids, brings myriad benefits, such as fast and accurate capturing of the microgrid dynamic behavior, and efficient and reliable operation of microgrids; however, they are prone to cyber disruptions which may cause critical social impacts and financial losses. Our vulnerability analysis proves that the cyber disruption, e.g., false data injection to microgrid control process, can deteriorate the control performance or even cause unstable operation. Therefore, it is imperative to design a resilient secondary control to mitigate these cyber disruptions. This dissertation proposes a two-layer Linear Quadratic Regulator (LQR) based controller in conjunction with a developed Unknown Input Observer (UIO) to address both cyber disruptions and uncertainties of renewable sources and load. In order to investigate the efficacy and scalability of the proposed control strategy, this controller is utilized for frequency control of a simple microgrid model as well as an inverter-based microgrid model. 


\section{Acknowledgements}

I wish to express my deep and sincere gratitude to my supervisor Prof. Jignesh Solanki for his guidance, support and encouragement in entire my Ph.D. program. Prof. Jignesh Solanki was always there to patiently listen and to give valuable advice. I will not forget his great feedback for enhancing my research presentation skills. Besides my supervisor, I cannot begin to express my thanks to Prof. Sarika Khushalani Solanki for participating in lengthy discussions to perfectly align my research and present my accomplishments. She has been very supportive and sympathetic in both my professional and daily lives. Working under these two great professors was a golden opportunity for me to leverage all their insightful comments and encouragements and ultimately enhance my academic qualification for my next chapter of life.

I would like to extend my sincere thanks to Prof. Muhammad Choudhry and Prof. Natalia Schmid, who gave me this fortunate to be my Ph.D. committee members. I personally learned from their professional behaviors and commitments and senses of responsibility. They gave me inspiration and immense knowledge of their areas of expertise within my Ph.D. program. I also very much appreciate Prof. Debangsu Bhattacharyya for kindly accepting to be as my external committee member although he is extremely busy.

I also would like to thank my colleagues, Deepak Tiwari, Hassan Ul Banna, Vishal Verma for the inspiring discussions, for the sleepless nights we were working together before deadlines, for having a friendly research environment, and for all the fun we have had in the last three and half years. Those days working with these friends will remain in my memory forever.

Last but certainly not the least, I would like to thank my family: my parents and to my brother for supporting me spiritually throughout my life and my kind wife who has helped me making this dissertation possible with her warm love, continued patience, and endless dedication. 


\section{Dedicated to my beloved wife and parents}




\section{Contents}

1 Introduction 1

Electric Vehicle Integration .....................................................................

Microgrid Control in the Presence of Uncertainties .....................................4

Cyber-Security Issues ................................................................................

2 Electric Vehicle Integration to Microgrids $\quad 7$

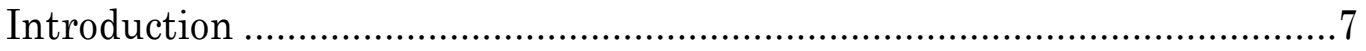

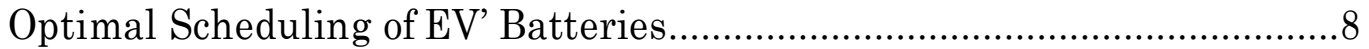

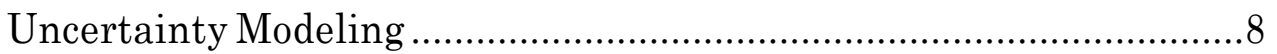

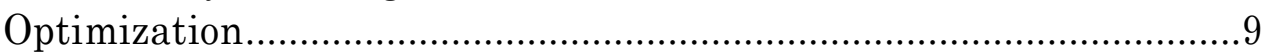

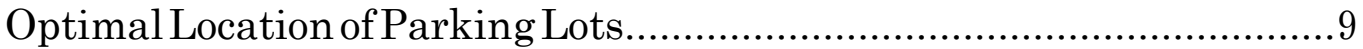

Simulation and Results ...............................................................................11

Obtaining Modified Daily Load Curve Using EV Scheduling 11

Finding the Best Parking Lots Using the Proposed Method- ologies 13

Conclusion

3 Artificial Intelligence-Based Control of Microgrids with Stochastic $\begin{array}{ll}\text { Elements } & 18\end{array}$

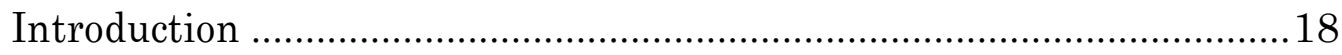

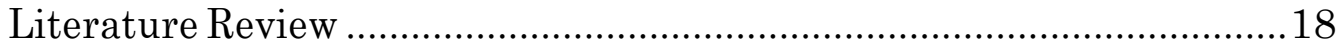

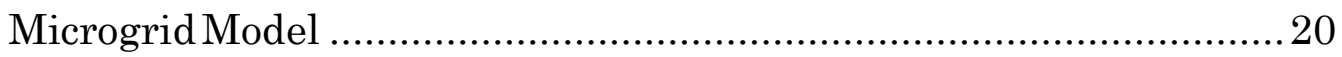

The State-Space Model of Microgrid................................................ 20

The Microgrid Control Structure ........................................................22

Hebb Learning Based Controller ................................................................23

Learning Methods for Weights................................................................24

Human Brain Emotional Learning Controller ............................................25

LFC Intelligent Control Based on Emotional Learning and its

Bi-Objective Control Structure ...................................................27

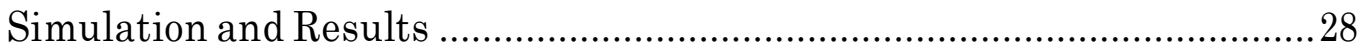

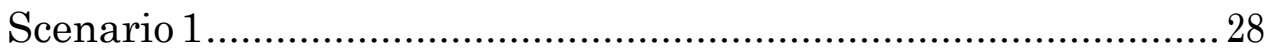

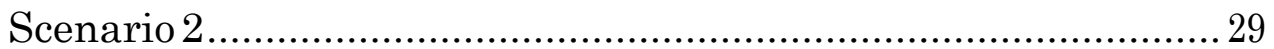

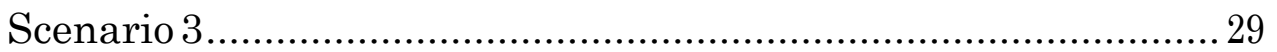

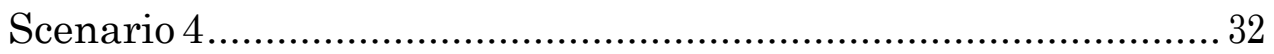

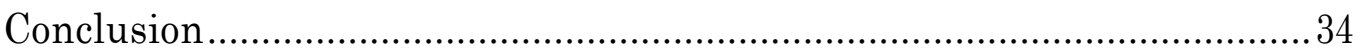


4 Resilient Control of Microgrids $\quad 35$

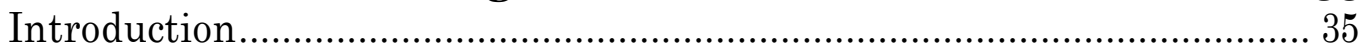

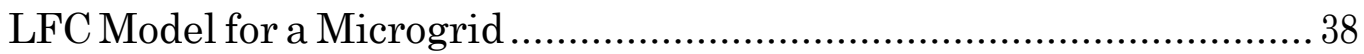

The State-Space Model of Microgrid.............................................. 38

The Microgrid Control Structure........................................................ 40

Vulnerability and Instability Analysis ......................................................... 40

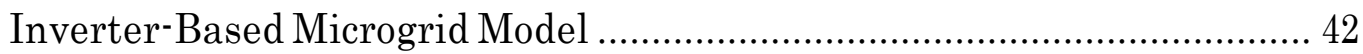

The Microgrid State-Space Representation ....................................... 42

Secondary Control of the Inverter-based Microgrid ............................ 46

The Proposed Control Method ................................................................ 46

Design of Stochastic Unknown Input Observer ................................. 46

Design of Unknown Input Compensator .............................................. 49

Stability Proof of the Proposed Methodology ......................................... 51

Simulation and Results............................................................................ 52

The Proposed Controller Robustness Under Uncertainties ................... 53

The Proposed Controller Resiliency Under FDI ................................... 60

The Proposed Controller Resiliency and scalability Performance Under FDI ................................................................64

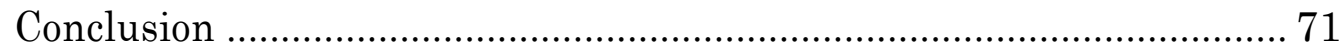

5 Conclusion $\quad \mathbf{7 4}$

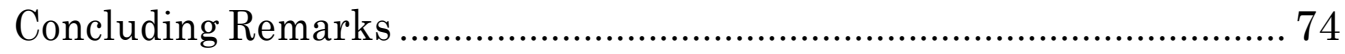

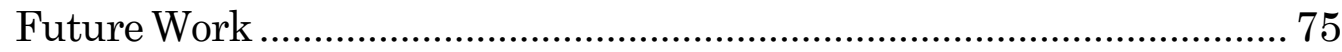




\section{List of Figures}

Smart grid structure VS legacy power system structure ...........................2

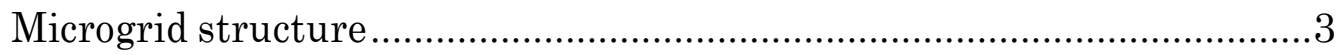

Deployment scenarios for the EV stock by 2030 ...................................

Data and power flow in an entire power system ...................................5

Available EVs in residential and non-residential parking in a day .......... 12

Charging and discharging pattern for EVs.............................................12

Actual and modified load after optimal scheduling of charging and

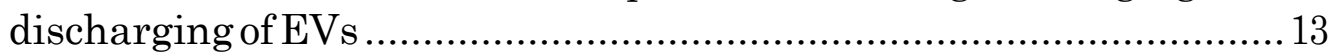

SOC patterns for all of the EV batteries in a day ......................................14

The case study; IEEE 13 node distribution system............................... 15

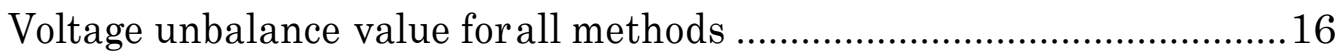

Power loss values for all methods ..................................................... 16

Simplified structure of the islanded microgrid .........................................20

The diesel generator and electric vehicle model in LFC of the microgrid 22

Diagram of Load Frequency Control.....................................................23

Diagram of a single neuron PID control system based on the Hebb

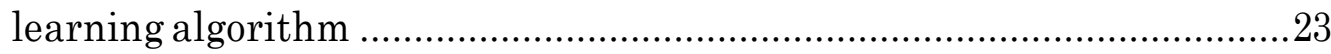

Detailed diagram of emotional learning system of the human brain .......25

Diagram of the proposed computational model of the human brain

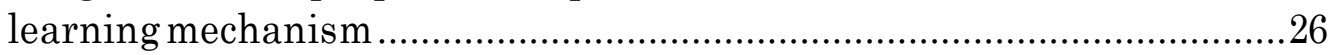

Diagram of Emotional Learning controller implemented in LFC .............. 27

Load and Wind Power Profiles ...........................................................28

Frequency deviation in Scenario 1 .......................................................29

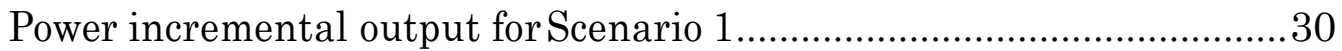

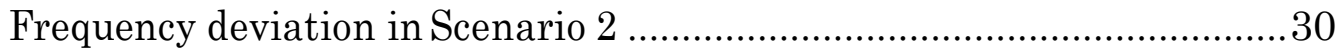

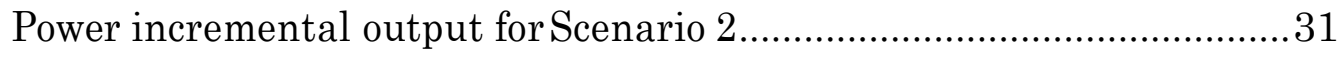

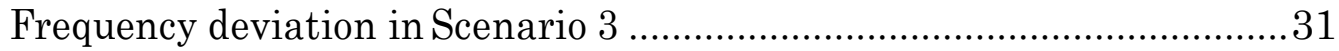

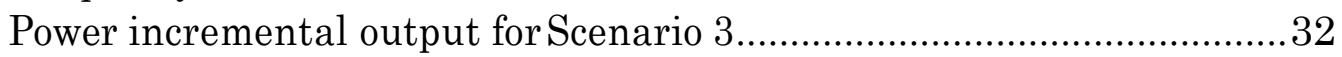

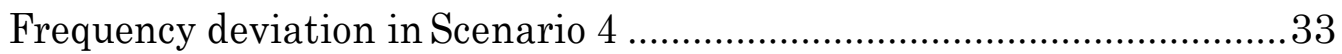

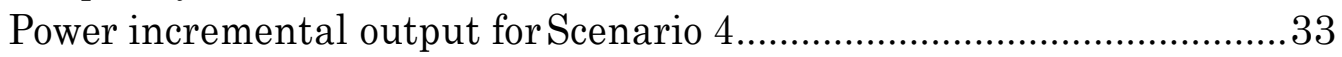

Cyber-Physical Power System Scheme of A Microgrid ...............................37

Diagram of Load Frequency Control........................................................... 39

The diesel generator and electric vehicle model in LFC of the microgrid 40 
Eigenvalues of the system for normal operation and under different

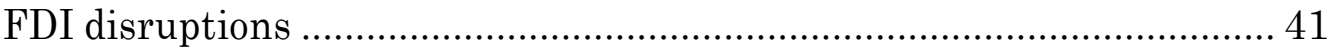

General diagram of DG inverter connected to Microgrids ......................... 43

External power controller diagram of DG inverter .................................... 43

Network topology of the inverter-based microgrid model ......................... 45

Load variation and wind power uncertainties (UI) ................................... 50

Load variation and wind power uncertainties (UI) .................................. 53

Frequency control response in case 1....................................................5 54

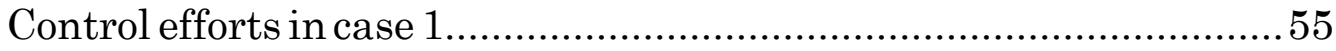

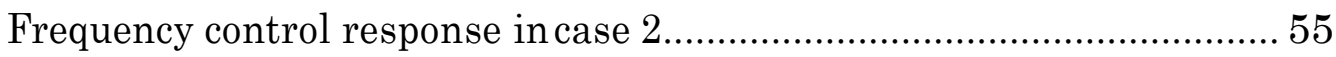

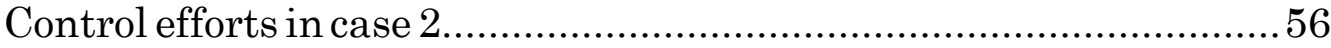

Frequency control response in case 3.................................................... 56

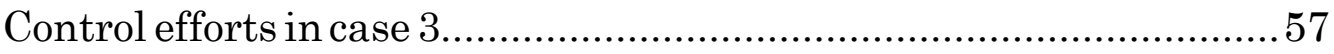

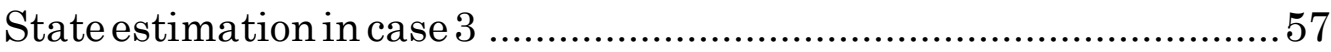

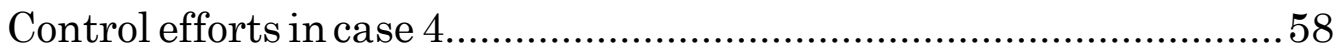

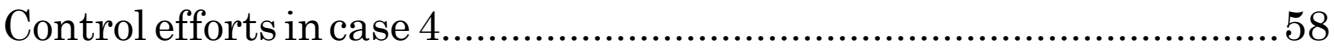

Frequency control response incase 5................................................... 59

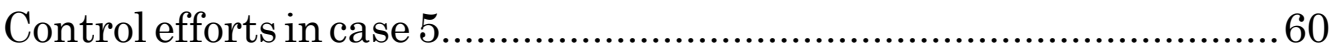

Frequency deviation and EVS control inputs in scenario 1.......................61

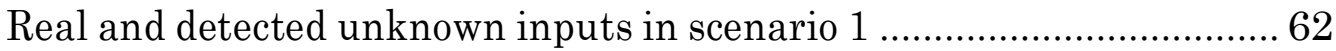

Frequency deviation and EVS control inputs in scenario 2....................... 63

Real and detected unknown inputs in scenario 2 ..................................... 63

Frequency deviation and EVS control inputs in scenario 3....................... 64

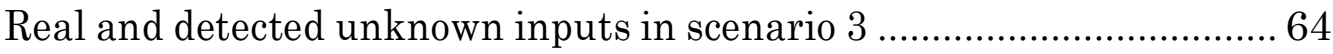

The Case Study: Microgrid topology ....................................................6 65

Second inverter angle $\delta_{2}$ in scenario 1 (Step load change)..........................67

Third inverter angle $\delta_{3}$ in scenario 1 (Step load change) ........................67

Second inverter frequency dynamic $\Delta f_{2}$ in scenario 1 (Step load change) 68

Third inverter frequency dynamic $\Delta f_{3}$ in scenario 1 (Step load change) 68

Second inverter angle $\delta_{2}$ in scenario 2 (FDI to the second inverter's

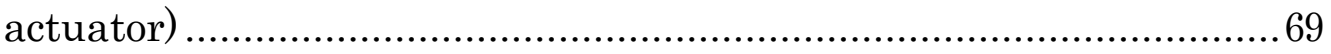

Third inverter angle $\delta_{3}$ in scenario 2 (FDI to the second inverter's

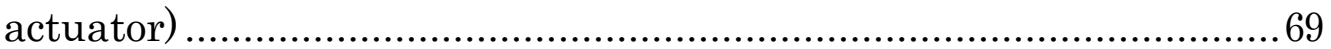

Second inverter frequency dynamic $\Delta f_{2}$ in scenario 2 (FDI to the second inverter's actuator) ....................................................................... 70

Third inverter frequency dynamic $\Delta f_{3}$ in scenario 2 (FDI to the sec-

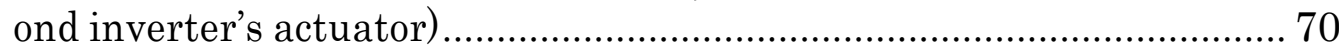

Second inverter angle $\delta_{2}$ in scenario 3 (Stealthy FDI to the second

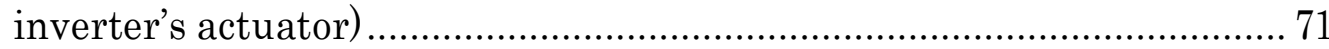

Third inverter angle $\delta_{3}$ in scenario 3 (Stealthy FDI to the second

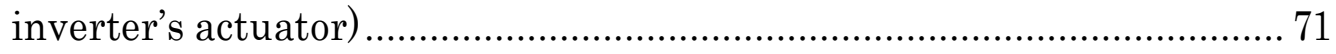

Second inverter frequency dynamic $\Delta f_{2}$ in scenario 3 (Stealthy FDI to the second inverter's actuator) ....................................................... 72 Third inverter frequency dynamic $\Delta f_{3}$ in scenario 3 (Stealthy FDI to the second inverter's actuator) ................................................................. 72 


\section{List of Tables}

Mean and variance for the simulation of an EV daily trip ...................... 9 The best node candidates considering objective functions separately and as multi-objective function...............................................................17

Load Frequency Control Tabular Survey ..............................................19

The parameters of this microgrid...........................................................21

ITAE Index Evaluation For All Controllers In All Four Scenarios ...........34

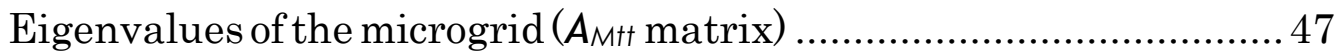

Control and estimation performances of controllers ...................................61

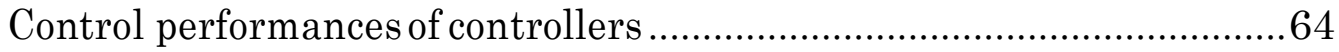

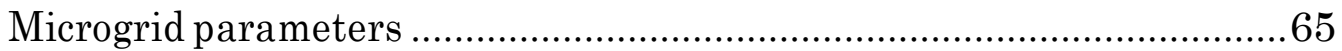

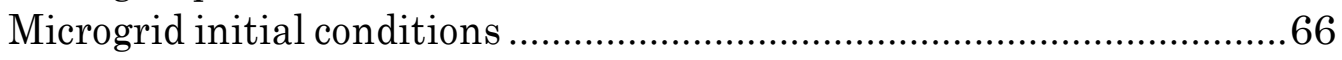




\section{List of Abbreviations}

DG Distributed Generations

RES Renewable Energy Sources

EV Electric Vehicle

IEA International Energy Agency

V2G Vehicle-to-Grid

G2V Grid-to-Vehicle

AI

Artificial Intelligence

PSO Particle Swarm Optimization

IoT Internet of Things

AMI Advanced Metering Infrastructure

DMS Data Management Systems

HAN Home Area Network

NAN Neighbouring Area Network

CPM Cyber-Physical Microgrid

LMI Linear Matrix Inequalities

FDI False Data Injection

MCS Monte Carlo simulation

SoC State of Charge

LFC Load Frequency Control

GA Genetic Algorithm

DMS Distribution Management System

MO Model Output

SI Stimulant Input

AO Amygdala Output

OCO Orbitofrontal-Cortex Output 
EC Emotional Control

PID Proportional-Integral-Derivative

ITAE Integral of Time0weighted Absolute Error

DoS Denial of Service

TDS Time Delay Switch

ELM Extreme Learning Machine

UIO Unknown Input Observer

SUIO Stochastic Unknown Input Observer

LQR Linear Quadratic Regulator

EVS Electric Vehicle Station

PMU Phasor Measurement Unit

VSI Voltage Source Inverters

UI Unknown Input

MAF Moving Average Filter

IAE Integral of Absolute Error

MAE Mean Absolute Error

LQG Linear Quadratic-Gaussian 


\section{Chapter 1}

\section{Introduction}

After the US northeast blackout incident in 2003 which affected 55 million people and cost $\$ 6$ billion, the Federal government and utilities have made significant investments in new technologies to modernize the grid and make it smarter. The investment occurred, which dedicated $\$ 9.5$ billion to increase the reliability and resiliency of the U.S. power system [1]. To make the gird smart or having "Smart Grid", we need to deploy advanced sensors, known as synchrophasors, which gives utilities better visibility into the health condition of the grid, and helps them respond more quickly to abnormal incidents. Smart Grids are the intelligent integration of electrical, information, and communication networks which facilitates control and management of the grid, including renewable resources efficiently. The main benefits of smart grid over legacy power systems are:

- Faster restoration of electricity after severe incidents

- Lower operation and management costs for both utilities, and consumers

- Higher integration of renewable energy sources and reduction of $\mathrm{CO}_{2}$ emission

- Better integration of customer-owned power generation systems, e.g. Electric Vehicles

- More reliable and resilient operation owing to the capability of a distributed configuration.

As shown in Figure 1.1, legacy power systems are mostly centralized which decrease reliability and resiliency of the grid exposed to extreme contingencies. Since the late 1990s, researchers in the United States and Europe started to probe decentralized ways to manage the integration of a large number of distributed energy resources and ultimately enhance reliability and resilience against cascading power failures, natural disasters, physical and cyber disruptions. "Microgrid" as smaller scale paradigm of the smart grid is created to locally provide the demand. Microgrids also perform isolated from the main grid to sustain critical services even in case of large-scale outages and failures [2]. The US Department of Energy defined Microgrids as "a group of interconnected loads and distributed energy resources 


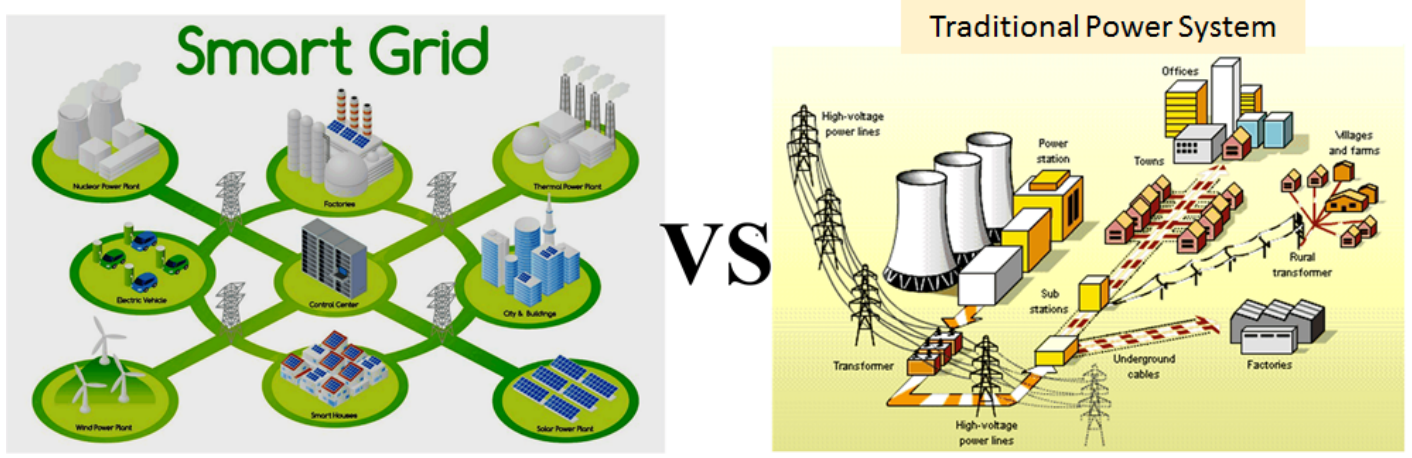

Figure 1.1: Smart grid structure VS legacy power system structure

within clearly defined electrical boundaries that acts as a single controllable entity with respect to the grid. A microgrid can connect and disconnect from the grid to enable it to operate in both grid-connected or island mode" [3]. The islanded mode of microgrids has been widely developed since it is more cost-effective than the grid-connected mode for some applications, including rural and distant areas. Microgrids are formed from the interconnection of distributed generations (DGs), energy storages, Electric Vehicles (EV), loads and communication structure as shown in Figure 1.2. In utility prospective, microgrids are independent energy systems that can operate in both islanded and grid-connected modes. Compared to legacy power systems, microgrids like smart grids utilize higher-level control configuration based on predefined communication and information protocols that involve coordination, monitoring, and integration and ultimately leads to more efficient, reliable and stable operations than standard distribution systems [4]. Microgrids can be islanded from the distribution systems and function as a small-scale grid while they can supply, store and locally distribute energy. Microgrids have been growing in number with applications of educational campuses, hospitals, military sites, large industrial complexes, small townships, and remote applications, such as telecom, rural households, etc. Another advantage of microgrids over legacy power systems is that they are easily able to locally harness renewable energy sources (RES), including wind power and photo-voltaic sources, through hierarchical control that aims to [5]:

- Increase renewable energy and other clean distributed generation integrated to the grid. The distributed energy sources often integrate into the grid through power-electronic interfaces. These interfaces can be easily controlled based on local exchanged information in a distributed environment not in a centralized way.

- Increase asset use through the integration of distributed systems and customer loads to reduce peak load and thus lower the costs of energy.

- Support achievement of renewable portfolio standards for renewable energy and energy efficiency. Enhance reliability, security, and resiliency from microgrid applications in critical infrastructure protection and highly constrained areas of the electric grid. 


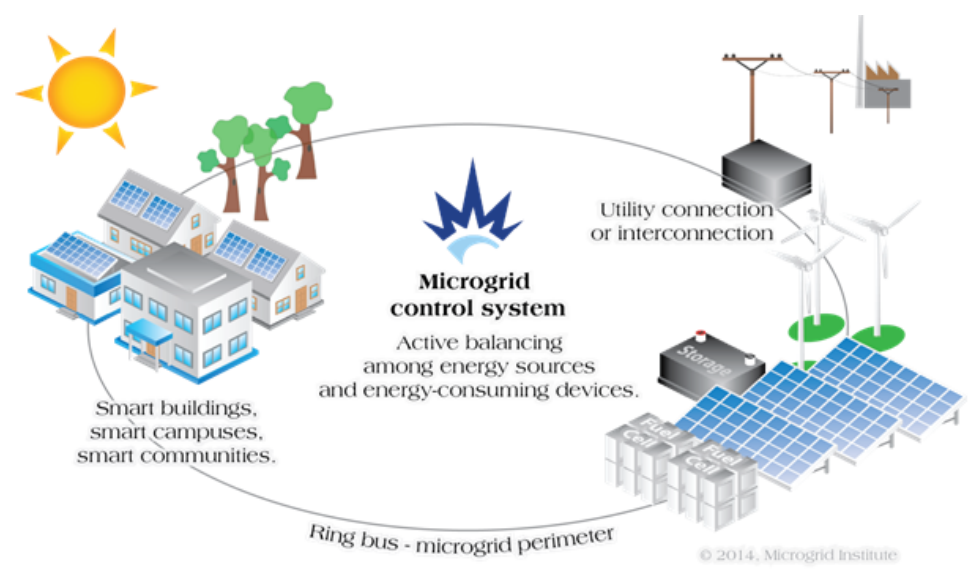

Figure 1.2: Microgrid structure

- Support reductions in oil use by enabling electric vehicle operations with the grid.

Although such efforts have been placed into the development of smart microgrids, the grid authorities are still facing three main challenges which include:

\section{Electric Vehicle Integration}

\section{Microgrid Control in the Presence of Uncertainties}

\section{Cyber-Security Issues}

\section{$1.1 \quad$ Electric Vehicle Integration}

Rapid decrease of battery costs and increase of their energy densities are the main reasons that are making EV industries more competitive compared to internal combustion engines and rapidly growing trend of EVs. Also, based on different scenarios and worldwide policies associated with EVs, the International Energy Agency (IEA) predicts some promising trends for EV deployment, ranging between 9 million and 20 million by 2020 and between 40 million and 70 million by 2025 shown in Figure 1.3 [6]. Hence it is necessary to study EVs' effects on microgrids and address their potential issues. Integrating EVs in microgrids can significantly decrease carbon emission.

Modern battery energy storage of electric vehicles can return electricity to microgrids, known as Vehicle-to-Grid (V2G) capability. V2G technology has drawn researchers' attention to leverage EVs for frequency control and flattening of daily load profile $[4,7,8]$. EVs have stable storage because their idle grid connection in parking lots is approximated to $90 \%$ of the day. Furthermore, due to large number of EVs and fast (dis)charging response of their storages, EVs can play a crucial role in quickly supplying the demand and keep the power balance between supply and load [9]. 


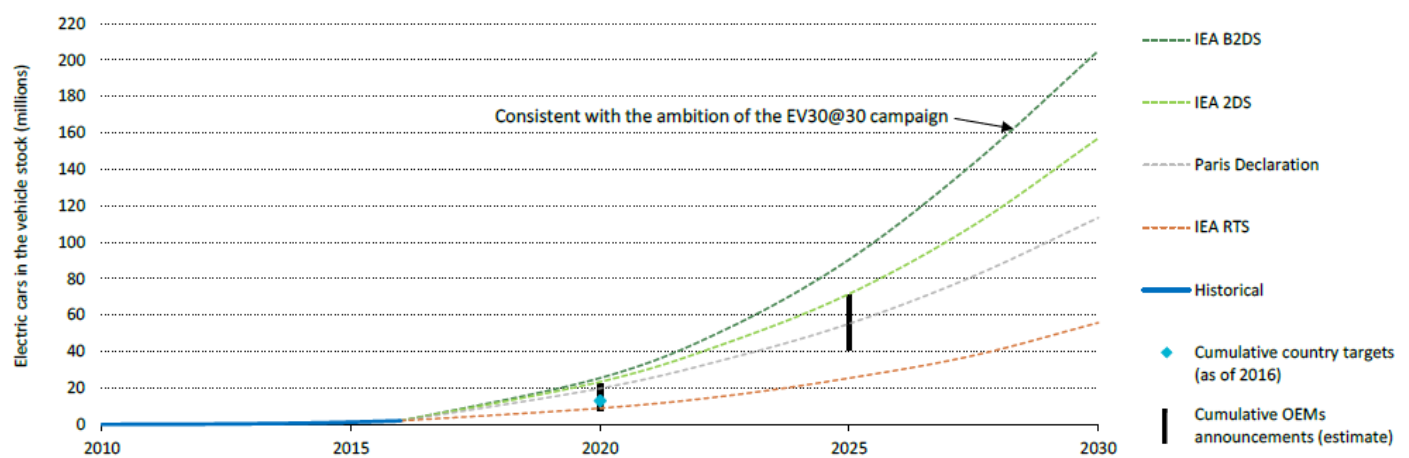

Figure 1.3: Deployment scenarios for the EV stock by 2030

Although EVs are very good alternatives to internal combustion engine cars, they will have significant impacts on microgrids. The total demand profile of power systems will be changed owing to the integration of EV charging and discharging. The charging of a large number of EVs can bring tremendous impacts on power systems. It is anticipated that the overall charging demand of the EVs (known as Grid-to-Vehicle or G2V) in the US can increase by $18 \%$ of the U.S. summer peak load in the presence of the EV penetration level of 30\% [10]. On the other hand, an EV can also provide energy to the power grid through discharging called as V2G. An optimal scheduling pattern of EV charging and discharging can effectively flatten the load profile of the distribution systems as well as lower potential capital and operational costs [11]. The smart EV integration into microgrids can improve the grids's reliability. Once EV charging process is optimally scheduled and controlled, it reduces loading of the grids' components and power loss and in turn regulates voltage and frequency [12]. Hence efficient EV integration has become a necessary step toward microgrids.

\subsection{Microgrid Control in the Presence of Uncer- tainties}

In addition to EV integration, researchers are concerned about addressing renewable energy uncertainties. To mitigate climate change and the green house effect, international policy is changing to massively deploy renewable energies. These energy sources, e.g., wind and solar energies, usually have intermittent behavior that produces power with uncertainties and perturbations. These uncertainties can degrade the microgrid control procedure and even lead to instability.

Identical to traditional power grids, the main dynamic control strategy for microgrids is the hierarchical control, including primary control (droop control) and secondary control. Primary control response is the immediate adjustment of power output by the governor or electronic controller to address any changes in the microgrid frequency or speed of rotating energy sources. Intermittent and low-inertia characteristics of renewable sources along with uncertainties in loads can degrade the 


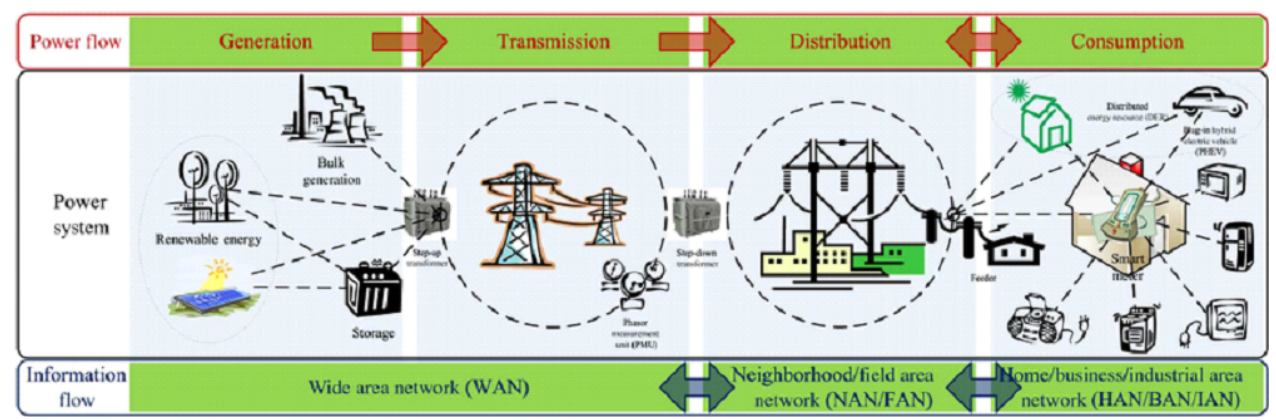

Figure 1.4: Data and power flow in an entire power system

primary control of microgrid to maintain the balance between supply and consumption that causes frequency deviation or even frequency instability. This frequency deviation issue is especially critical for an islanded microgrid that does not have frequency and voltage support from the main grid. Due to improving primary control's response and properly employing other energy sources than governor-based or droop-based energy sources (e.g. batteries), secondary control is adopted for microgrid control. Designing an effective secondary control method is vital for islanded microgrids. Applying secondary control based on Artificial Intelligence (AI) is a new solution to explore. These methods, e.g., general type II fuzzy logic [13], neural network and Particle Swarm Optimization (PSO) algorithm [14], usually do not require the microgrid model to cope with the microgrid uncertainties. Since AI-based secondary control works based on intuitive response and expert learning systems. In other words, they do not depend on the microgrid model and usually microgrid exchanged information to properly operate microgrids. Hence proposing secondary control based on an analytical control method is another solution to encounter microgrid uncertainties.

\subsection{Cyber-Security Issues}

Using analytical control methods, a supervisory secondary control utilizes measurements and communication systems to guarantee frequency deviation is eliminated. In modern secondary control, two-way communication links enabled by the Internet of Things (IoT) are deployed through smart meters to facilitate energy delivery and management. In distribution systems and microgrids, Advanced Metering Infrastructure (AMI) is utilized to dynamically capture the data samples with smart meters, store and send them to Data Management Systems (DMS) to be analyzed. These collected data are sent to DMS using Home Area Network (HAN). Communication among neighbouring HANs is termed as Neighbouring Area Network (NAN). The energy and data flow in an entire power system is shown in Figure 1.4.

This tight interaction of information and communication networks with power distribution system components in microgrids creates a new architecture of energy systems called Cyber-Physical Microgrid (CPM). Leveraging the cyber-interfaced secondary control, we can optimally resolve the unbalance between supply and load 
by properly adjusting operating set-points of back-up sources, such as energy storage systems. Considering the costly operation of the storage system, it may not be the best option for compensating this power shortage of islanded microgrids [15]. As discussed earlier, EV batteries can be applied for this purpose.

All microgrid components, including EVs and distributed energy resources, must be connected through wireless and/or mobile communication networks and relative protocols applied in IoT communications, such as low-cost protocol of IEEE 802.15.4x (e.g., ZigBee) and more secure protocol of IEEE 802.11.x (e.g., WiFi, HAN, and NAN). Despite the myriad advantages of communication and cyber interface integrated into smart grids, it makes the system vulnerable to many types of cyber disruptions. These security issues are not arisen only from the integration of low-cost and low-power wireless protocols, e.g., ZigBee, into microgrids. It is seen that even more secure protocols, e.g., WiFi, in a HAN or a NAN network, can be severely targeted by adversaries [16]. These cyber manipulations can cause permanent equipment impairment and cascading outages leading to blackouts. This, in turn, causes adverse social impact and economic losses asserting the importance of such studies.

Secondary control based on analytic approaches heavily depends on cyber and communication networks that can make it very prone to cyber manipulations. Hence these secondary control methods must be resiliently designed. There are many model-based or analytic approaches,e.g., distributed-averaging proportional-integral [17], model-predictive control [18], and Linear Matrix Inequalities (LMI) based controller [19], leveraged for frequency and voltage control of microgrids.

Considering these critical challenges, we were persuaded to resolve them for microgrids. In summary, this dissertation makes the following contributions organized in three chapters investigating the current challenges in microgrids and one more chapter describing the concluding remarks and the potential future work.

- Chapter 2 investigates efficient strategies for EV integration to microgrids. In this chapter, first an optimal charging and discharging schedule for EVs is obtained; then the best locations for EV parking lots in a microgrid, which is ieee 13 node test feeder, are found to optimize both power loss and voltage unbalance.

- Chapter 3 presents two self-tuning controllers based on Artificial intelligence techniques, human brain emotional learning and Hebb learning, to control the frequency of microgrids in the presence of uncertainties arising from load variation and wind power fluctuations.

- Chapter 4 designs a resilient observer-based controller against cyber manipulation (False Data Injection) for the islanded microgrid. Also, this controller can well perform in the presence of stochastic behavior of wind and load profiles. In order to show the scalability of this proposed method, it is applied for a larger state-space model, which is the inverter-based microgrid model.

- Chapter 5 explains the conclusions and future work. 


\section{Chapter 2}

\section{Electric Vehicle Integration to Microgrids}

\section{$2.1 \quad$ Introduction}

With the rising trend for manufacturing and using EVs all over the world, it is interesting to study their effects on power distribution systems. The registered sales of EVs in California were more than 200,000 units in March of 2016 [20] with higher sales in the Netherlands too, which was the forerunner of EVs in Europe in 2013 [21]. A single EV penetration does not affect the operation of a distribution system; however, an aggregator of EVs has a large impact. Therefore, they are usually aggregated and then their influence, as demand or energy source, is investigated on the grid [22]. EVs can be operated in charging and discharging modes. It is important to comprehensively model EVs to consider both operating modes. In the past, deterministic methods have been applied to model the uncertainty of EVs. However, considering the uncertain behavior of EVs, probabilistic methods are needed. There are many probabilistic methods. The most comprehensive method is Monte Carlo simulation (MCS) $[23,24]$ that can be divided into non-sequential and sequential methods. The former is easier to apply but does not consider the chronology of the process as against the latter. R.C. Leau et al. [25] use Roulette wheel selection and Monte Carlo simulations for modeling EV's uncertainties in reinforcement planning problem of distribution systems. MCS for optimal integration of EV aggregators in the presence of uncertainty of photovoltaic is considered in [26]. As another sequential technique, the decision process is used in [27] to model the uncertainty. In [28], dynamic programming and game theory is applied to optimally manage the charging schedule of electric vehicles to smooth out the load curve whereas demand responsive load for the same objective is proposed in [29].

Once the EVs are accurately modeled, their effects on distribution systems can be analyzed. Some researchers in [30-34] evaluate solutions for power quality problem with EVs. In this work, we model optimal charging and discharging and evaluate the best location for power quality. S. Gao et al [30] worked on power loss in EV. In [31], the authors present a multi-objective algorithm to optimally determine parking lots 
number to be allocated in a distribution system. Shahnia et al. [32] considered voltage profile. Using a genetic algorithm, the proper place for EV parking lots and optimum capacity for each parking were found to reduce the power loss in [33]. In [34], charging coordination is done based on the line flow limits and power loss. Harmonic problems due to EVs are investigated in [35].

In this work, at first, using sequential MCS and PSO algorithm, the optimum charging and discharging of EV aggregators is obtained. Then, the best location for parking lots is determined. Section II discusses the optimal scheduling of EV's batteries. In section III, the procedure for achieving the optimal parking lots for EV aggregators is introduced. Simulation and results are discussed in Section IV.

\subsection{Optimal Scheduling of EV' Batteries}

\subsubsection{Uncertainty Modeling}

The uncertain behavior of EVs cannot be modeled using deterministic algorithms. The uncertainties come from the fact that the start time of charging and discharging of EVs are unknown. Also, daily routs of EVs in one specific area have stochastic behaviors. In order to model the uncertainties, truncated normal distribution function [36] as shown in equation 2.1 [22] is used a random variable for limiting the range of generated EV uncertainty pattern to 24 hours.

$$
f(x, \mu, \sigma, a, b)=\frac{\frac{1}{\delta} \phi\left(\frac{x-\mu}{\sigma}\right)}{\phi\left(\frac{b-\mu}{\sigma}\right)-\phi\left(\frac{a-\mu}{\sigma}\right)}
$$

where $a$ and $b$ are the maximum and minimum values of each random variable, $\phi$ is a notation for normal distribution function and $\mu$ and $\sigma$ are the mean and standard deviation of the variables. Here, sequential MCS is applied for simulating over 24 hour period. This MCS works as follows [37]:

1. Generating all uncertainties based on Truncated Normal Distribution.

2. Utilizing the PSO algorithm to obtain the optimized decision variables.

3. Obtaining the load pattern for a period of 24 hours.

4. Checking the stopping criterion of the simulation: 4-1) If the criterion is satisfied, this loop is finished and the algorithm goes to the next particle of PSO; 4-2) If the criterion is not satisfied, the algorithm goes to the first step of the sequential Monte Carlo Simulation.

In order to obtain detailed information about PSO, [38] can be referred. For each case, the simulation is terminated when its coefficient of variation reaches a predefined minimum value. The values for mean and standard deviation of each variable for EVs are as shown in Table 2.1 [22]: 
Table 2.1: Mean and variance for the simulation of an EV daily trip [22]

\begin{tabular}{|l|l|l|l|l|l|}
\cline { 2 - 6 } \multicolumn{1}{c|}{} & $\begin{array}{l}\text { Departure } \\
\text { Time }\end{array}$ & $\begin{array}{l}\text { Parking } \\
\text { Period }\end{array}$ & $\begin{array}{l}\text { Commuting } \\
\text { Time }\end{array}$ & $\begin{array}{l}\text { Commuting } \\
\text { Time }\end{array}$ & Storage \\
\hline Mean & $7: 15 \mathrm{AM}$ & $9 \mathrm{~h}$ and 20Min & $30 \mathrm{Min}$ & $25 \mathrm{Km}$ & $20 \mathrm{kWh}$ \\
\hline Variance & $50 \mathrm{Min}$ & $50 \mathrm{Km}$ & $15 \mathrm{Min}$ & $15 \mathrm{Km}$ & $5 \mathrm{kWh}$ \\
\hline
\end{tabular}

\subsubsection{Optimization}

The fitness function for optimizing the charging and discharging pattern of the EVs is as defined in equation 2.2:

$$
J=C_{p} \times N_{h}^{2}+C_{o p} \times N_{L}^{2}
$$

where $C_{P}$ is the maximum load, $C_{O P}$ is the minimum load, $N_{h}$ is the sum of the hours when the load is more than the peak shaving percentage $\left(X_{P} \%\right), N_{L}$ is the sum of the hours when the load is less than the valley filling percentage $\left(X_{O P} \%\right)$ [36]. Penalty factor is considered to ensure the satisfaction of charging constraints as given by equation $2.3[22]$.

$$
J=C_{p} \times N_{h}^{2}+C_{o p} \times N_{L}^{2}+P \times C_{S O C}
$$

where $P$ is penalty factor and $C_{S O C}$ is the cost of the state of charging. The charge and discharge rates of the EV batteries satisfy the following inequality constraints as in 2.4:

$$
\begin{aligned}
& S O C_{\min } \leq S O C^{i} \leq 1 \\
& C h_{\text {rati }}^{\min } \leq C h_{\text {rate }} \leq C h_{\text {rate }}^{\max } \\
& D h_{\text {rate }}^{\text {min }} \leq D h_{\text {rate }} \leq D h_{\text {rate }}^{\text {max }}
\end{aligned}
$$

$S O C_{\min }$ is the minimum value for SOC. In this problem, it is $40 \%$ to let the vehicles continue their travel. Also, $C h_{\text {rate }}^{\min }$ and $D h_{\text {rate }}^{\min }$ are the minimum charge and discharge rates and $C h_{\text {rate }}^{\max }$ and $D h_{\text {rate }}^{\max }$ are the maximum charge and discharge rates. During the hours in which the load is more than $X_{P} \%$, EVs can be discharged with $D h_{\text {rate }}$. In addition, when the load is more than $X_{O P} \%$, EVs can be charged with $C h_{\text {rate }}$. MCS is utilized for uncertainty simulation within each iteration of PSO. A 24-hour curve of EV daily movement is extracted by taking an average of all MCS iterations.

\subsection{Optimal Location of Parking Lots}

Since all of power system problems are more severe and critical in peak hours, the best placement of parking lots is needed to be determined. For the optimal location, voltage unbalance and power loss objectives are considered. At first, this problem is solved considering single-objective optimization and then, a multi-objective optimization solution is obtained considering both objectives. For computing the voltage 
unbalance, equation 2.5 from [39] is used:

$$
V_{\text {unbalance }}=\frac{\mid \text { Maximum Devision from Average } \mid}{\left|V_{\text {average }}\right|} 100 \%
$$

The single-objective fitness functions are given by equation 2.6-2.7, where voltage unbalance is the deviation of voltage from the average voltage and $P_{\text {loss }}$ is the total power loss of the system. Fitness function for this optimization algorithm is a summation of voltage unbalances for all nodes as below:

$$
J_{1}=\sum_{i=1}^{\text {bus number }} V_{\text {unbalance }, i}
$$

For calculating the power loss, the absolute difference value of active power for both sides of each line is considered. The fitness function for that is mentioned below:

$$
J_{2}=\sum_{j=1}^{\text {line number }} P_{\text {Loss }, j}
$$

where $P_{l o s s, j}$ is power loss for $j^{\text {th }}$ of distribution line. To solve the multi-objective problem, including both voltage imbalance and power loss, each fitness function is fuzzified. Due to the dissimilarity of fitness functions, a multi-objective optimization algorithm based on fuzzification of these objectives is utilized [40]. The triangle fuzzy membership function for fuzzifying the objects is shown in equation 2.8. In this equation, Object $_{\max }$ and Object $_{\min }$ are respectively maximum and minimum defined values for the object (object is a specific objective function). Also, the new fuzzified objective function is given by equation 2.9 with weighting factors of $w_{1}$ and $w_{2}$ associated with fuzzy membership functions $\mu_{\text {loss }}$ and $\mu_{U V}$ [37]:

$$
\begin{aligned}
& \mu_{\text {object }}=\left\{\begin{array}{cl}
\text { Object }_{\max }-\text { Object }_{\text {Object }} \max -\text { Object }_{\min } & \text { Object }_{\min } \leq \text { Object } \leq \text { Object }_{\max } \\
1 & \text { Object } \leq \text { Object }_{\min } \\
0 & \text { Object } \geq \text { Object }_{\max }
\end{array}\right. \\
& J=w_{1} \times \mu_{\text {loss }}+w_{2} \times \mu_{U V}
\end{aligned}
$$

Other constraints to keep the balance between the active power generated and the load and loss, to keep reactive power generated and reactive power of demand and loss, to consider the maximum and minimum value of voltage for each bus and maximum permitted power flow on each line are also considered. For all of these three optimization algorithms, constraints of load flow, the power balance between load and generation and loading limitations [36] are considered as below: 


$$
\begin{gathered}
P_{g}+\sum_{i=1}^{N} P_{V 2 G}^{i}=P_{D}+P_{\text {Loss }} \\
Q_{g}=Q_{D}+Q_{\text {Loss }} \\
V_{i}^{\min } \leq V_{i} \leq V_{i}^{\max } \\
\left|I_{j}\right| \leq\left|I_{j}\right|^{\max }
\end{gathered}
$$

where $P_{g}, P_{D}, P_{\text {Loss }}$, and $P_{V 2 G}$ are total power supply, total active demand, total power loss in the grid, and power exchanged between vehicle batteries and the grid respectively. Also, $Q_{g}, Q_{D}$, and $Q_{\text {Loss }}$ are total reactive power supply, total reactive demand, total reactive power loss in the grid, and reactive power exchanged between vehicle batteries and the grid respectively. A multi-objective optimization can be solved using the analytical optimization algorithms [41] however, in this case, obtaining and leveraging the system model is difficult. PSO optimization based on the Pareto frontier has been used to solve Multi-objective problems [42,43]; however, this algorithm has a large computation and complexity. Due to the discrete space of this optimization problem, binary PSO algorithm is used in this work. First, the initial population of EV locations is randomly generated, and then the algorithm proceeds to move to the best solution using equations (14) and (15). $V_{i}$ and $X_{i}$ are velocity and position of $i^{\text {th }}$ solution candidate (or particle in PSO). $\omega, \alpha_{1}, \alpha_{2}$, $R_{1}$, and $R_{2}$ are converging coefficients which adjust weights for movement inertia, individual and swarm movements of particles toward the optimal solution in PSO algorithm.

$$
\begin{gathered}
V_{i}^{k+1}=\omega V_{i}^{k}+\alpha_{1} R_{1}\left(X_{\text {Pbest }, i}^{k}-X_{i}^{k}\right)+\alpha_{2} R_{2}\left(X_{\text {Gbest }, i}^{k}-X_{i}^{k}\right) \\
X_{i}^{k+1}=X_{i}^{k}+V_{i}^{k+1}
\end{gathered}
$$

\subsection{Simulation and Results}

\subsubsection{Obtaining Modified Daily Load Curve Using EV Schedul- ing}

Here hourly load values of last day of annual load model for IEEE RTS system are utilized and $100 \mathrm{EVs}$ are considered. The number of available EVs parked in residential and official (non-residential) parking lots, which is obtained using MCS, is depicted in Figure 2.1. The optimized charging and discharging obtained from MCS is shown in Figure 2.2 which indeed shows the exchanged power from residential and official parking lots to the grid after the optimization.

The hourly load behavior before and after optimization is shown in Figure 2.3. 


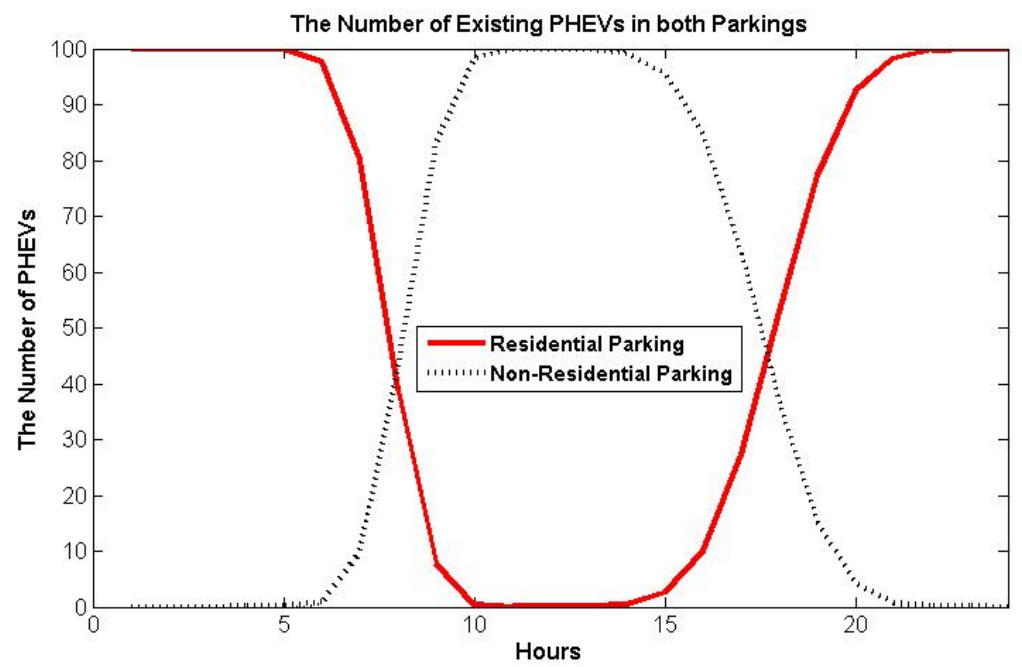

Figure 2.1: Available EVs in residential and non-residential parking in a day

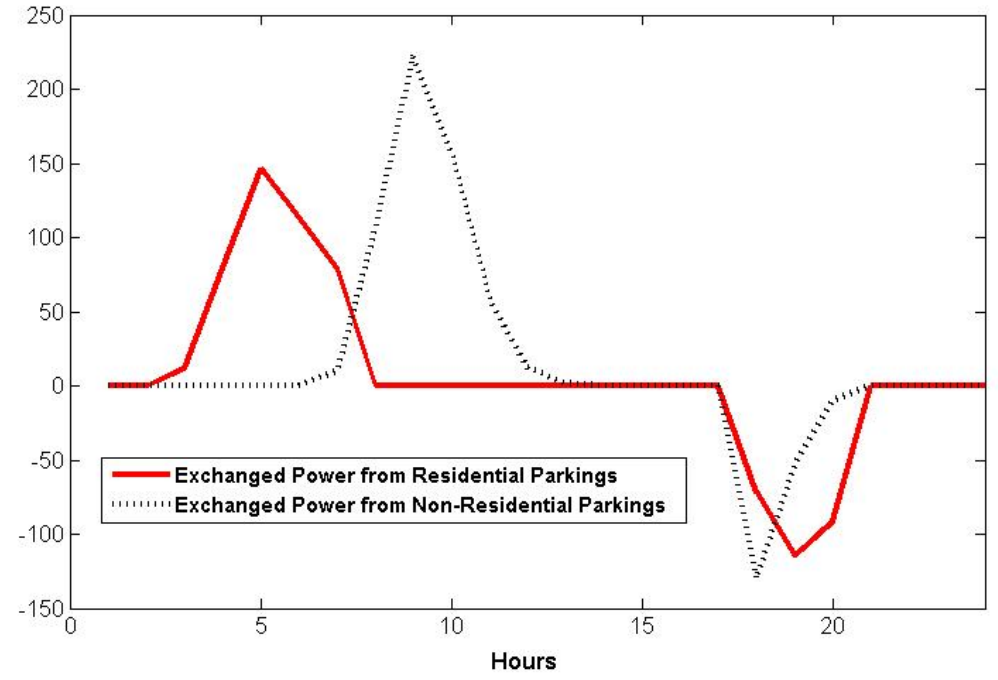

Figure 2.2: Charging and discharging pattern for EVs 


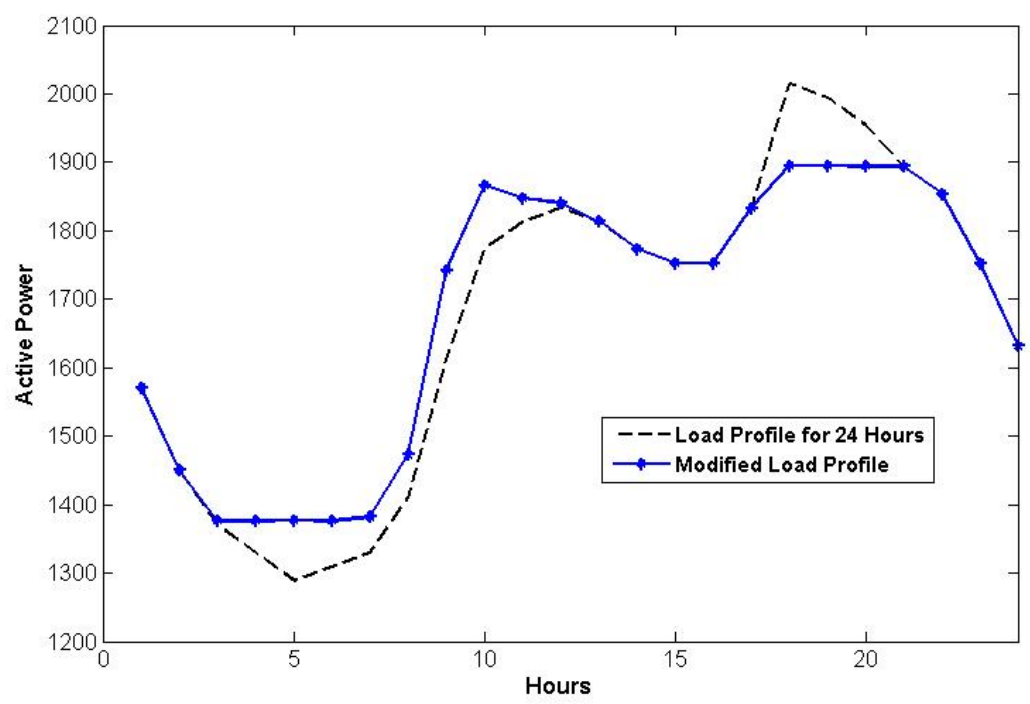

Figure 2.3: Actual and modified load after optimal scheduling of charging and discharging of EVs

Since the vehicles need to continue their trips between residential and administrative areas, their SOC\% must be more than $40 \%$ every time in a day. Using the proposed method, this important constraint is satisfied for all hours of the day. Figure 2.4 demonstrates SOC patterns for batteries of all EVs. Considering this figure, it is confirmed that all of the batteries have $40 \%$ of their stored energy during a day.

It is clear that the peak hour shifts from 18 to 21 after the optimization so there is a new peak hour after the optimization. Peak hour is a critical condition for distribution power grids; therefore, operation in this time is really important for utilities. Hence, the utilities should know when the peak hour for their grids occurs. In this research, it is shown that peak hour changes to the other time when charging and discharging procedure of EVs is optimized. Therefore, this new peak hour must be considered in studies related to peak hour.

\subsubsection{Finding the Best Parking Lots Using the Proposed Methodologies}

To find the best parking lot, IEEE 13 node system is simulated in MATLABSIMULINK. We seek to find locations of two parking lots, one residential and one administrative. The capacity of each of them is equal to 170 kilowatts. As shown in Figure 2.5, there are seven candidates of locations for each of residential and administrative parking lot. All phases for bus numbers 646, 645, 633 are considered as location candidates for the residential region. Also, all phases for bus numbers $652,611,684$, and 680 are considered as location candidates for the administrative region.

As mentioned in the previous section, each objective function of the total power 


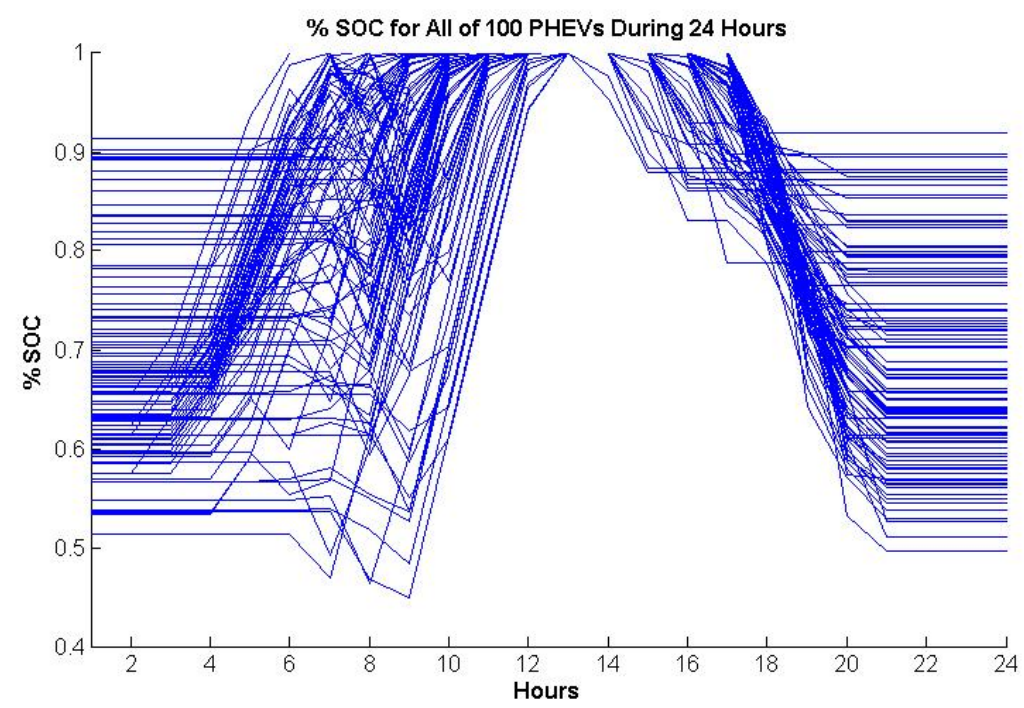

Figure 2.4: SOC patterns for all of the EV batteries in a day

loss and voltage unbalance is considered separately and also together as the proposed multi-objective optimization problems. Figure 2.6 compares the voltage unbalances using single-objective algorithms individually and the multi-objective algorithm. Similarly, Figure 2.7 compares the total power loss of the grid using single-objective algorithms individually and multi-objective algorithm. Power loss computation does not include loss contributions from transformers and the regulating transformer.

As seen from Figures 2.6 and 2.7, for each node and line, voltage unbalance and power loss is lower when the EV aggregators are in the locations determined by the optimization algorithms. Table 2.2 provides the proposed placement of EV aggregators, considering power loss and voltage unbalance.

As seen from Table 2.2, voltage unbalances, and power loss is lower when the optimum locations for parking lots are provided. Also, considering the problem with both objectives together that is multi-objective problem provides better solutions even though some of the locations overlap with those of single objective techniques.

\subsection{Conclusion}

This work has two parts. First part of this research is focused on finding the optimal scheme of charging and discharging for batteries of EVs. To do so, sequential MCS and an optimization algorithm are applied. At first, the stochastic behavior of daily trips of EVs is modeled, and then its chronological nature is completed using sequential MCS. The optimization algorithm is applied to provide the best pattern of charging and discharging for EVs so that peak load is shaved and the valley of that is filled. In simple terms, the objective function in the first part is smoothing out the daily load pattern. In the second part, the best parking lots are suggested using optimization algorithms, either single-objective or multi-objective based. In order 


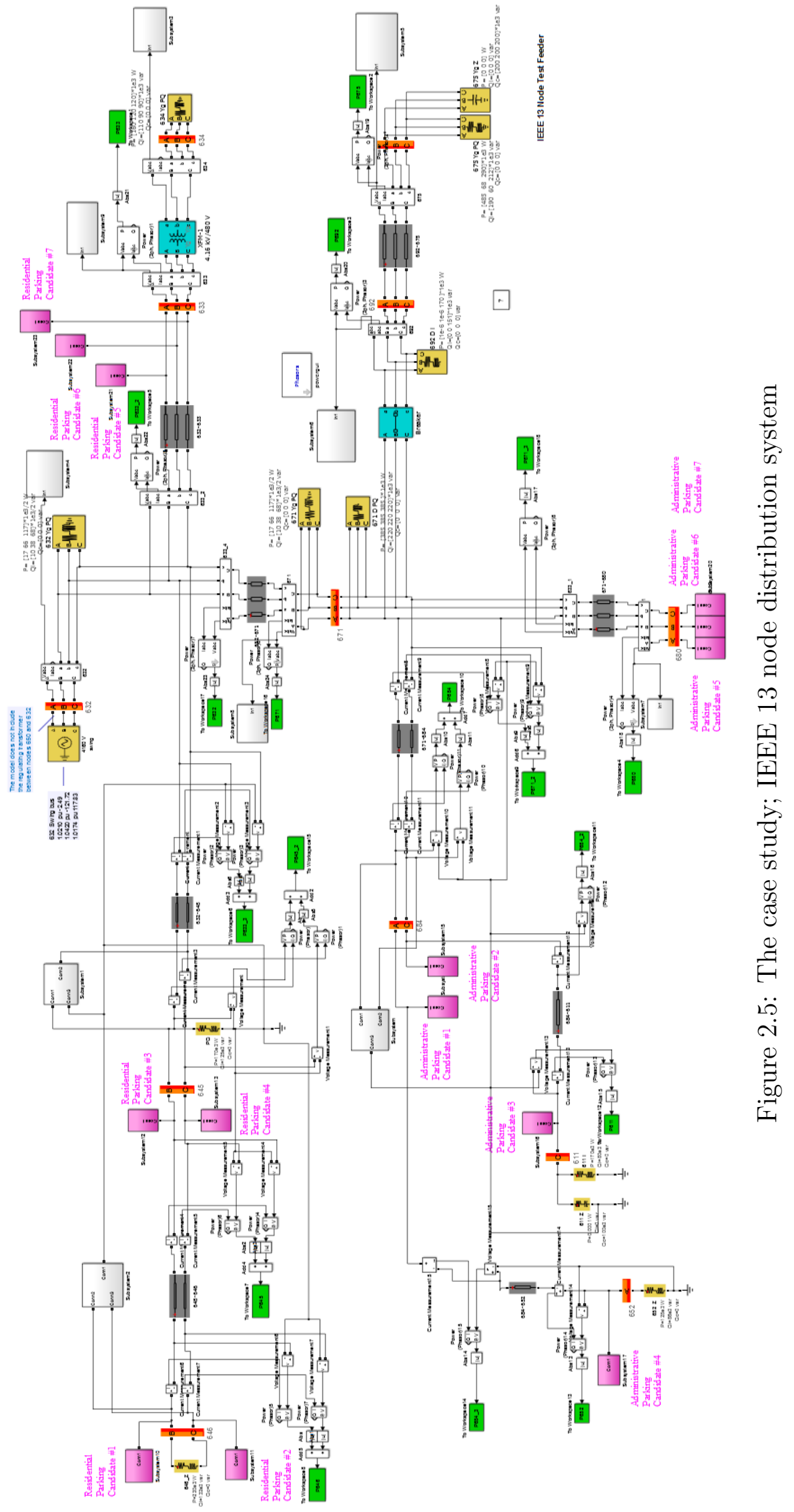




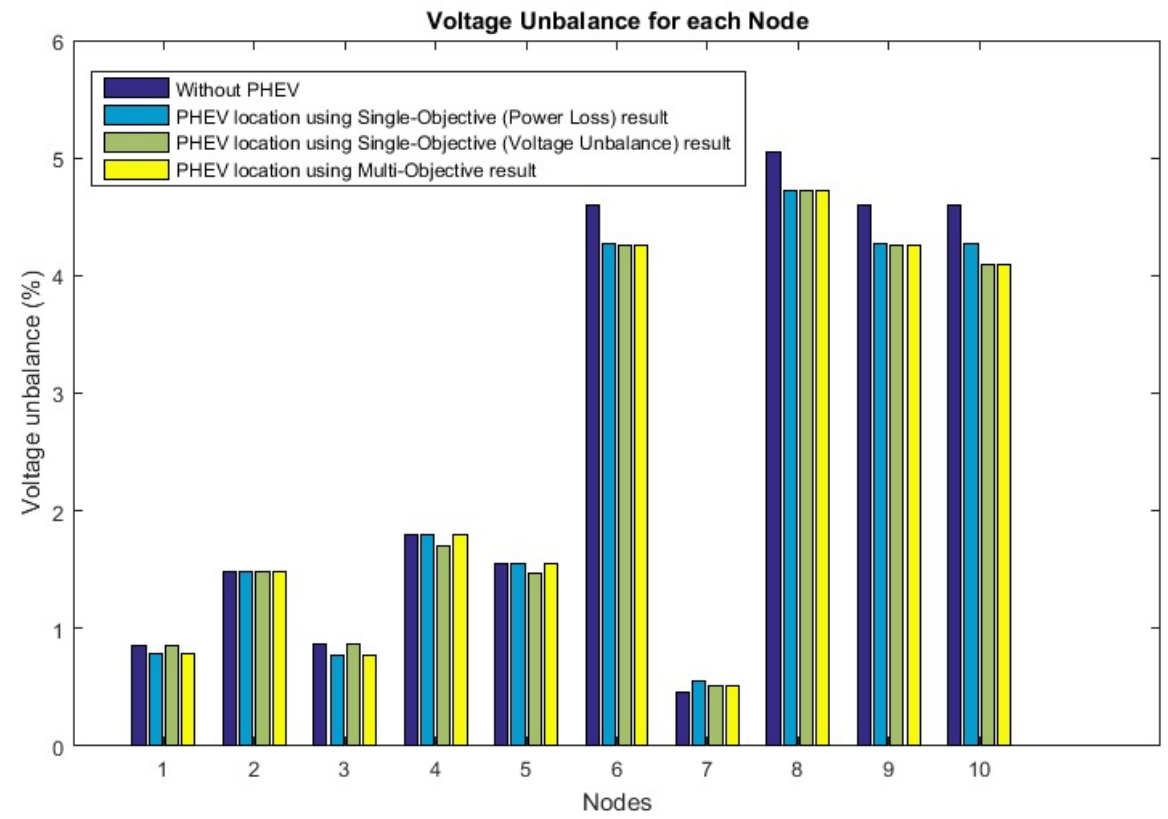

Figure 2.6: Voltage unbalance value for all methods

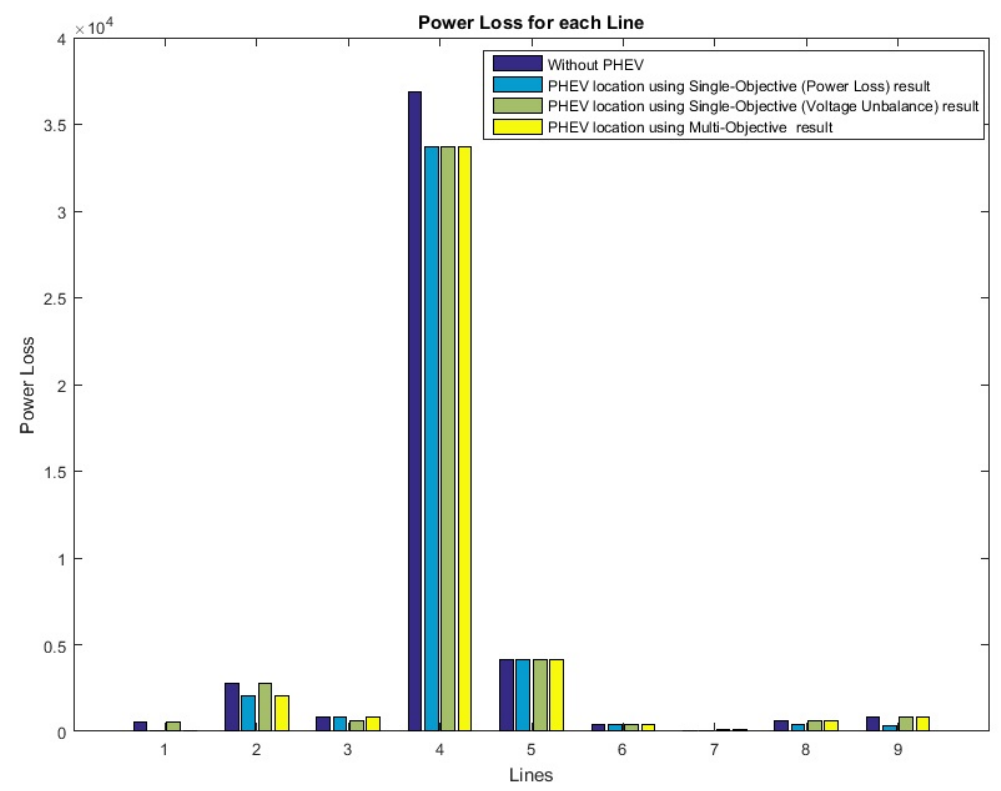

Figure 2.7: Power loss values for all methods 
Table 2.2: The best node candidates considering objective functions separately and as multi-objective function

\begin{tabular}{|c|c|c|c|c|}
\cline { 2 - 5 } \multicolumn{1}{c|}{} & $\begin{array}{c}\text { Without } \\
\mathrm{EV} \\
\text { aggregators }\end{array}$ & $\begin{array}{c}\text { Single- } \\
\text { Objective } \\
\text { based on } \\
\text { voltage } \\
\text { unbalance }\end{array}$ & $\begin{array}{c}\text { Single- } \\
\text { Objective } \\
\text { based on } \\
\text { power loss }\end{array}$ & $\begin{array}{c}\text { Multi- } \\
\text { Objective } \\
\text { optimization }\end{array}$ \\
\hline $\begin{array}{c}\text { Residential } \\
\text { Parking lot }\end{array}$ & - & 5 & 2 & 2 \\
\hline $\begin{array}{c}\text { Administrative } \\
\text { Parking lot }\end{array}$ & - & 5 & 4 & 5 \\
\hline $\begin{array}{c}\text { Voltage } \\
\text { unbalance (\%) }\end{array}$ & 25.85 & 24.23 & 24.44 & 24.22 \\
\hline Power Loss (W) & $4.69 \mathrm{E}+04$ & $4.37 \mathrm{E}+04$ & $4.19 \mathrm{E}+04$ & $4.26 \mathrm{E}+04$ \\
\hline
\end{tabular}

to propose a good operating point, voltage unbalance and power loss during peak hour are considered as optimization objectives. The results can show that power loss and voltage unbalance are less when these algorithms are applied. Hence, it can be noted that using an optimal location of EVs; we can improve the system operation point regarding voltage unbalance and power loss problems. 


\section{Chapter 3}

\section{Artificial Intelligence-Based Control of Microgrids with Stochastic Elements}

\subsection{Introduction}

The microgrid frequency can be controlled effectively when the generation and load are balanced. Microgrids can operate in two working modes, grid-connected and islanded. The latter has more critical Load Frequency Control (LFC) than the former because of the limited resources to fulfill demand. Also, there are many distributed generations in an islanded microgrid that have stochastic and uncertain behaviors, such as wind turbines, photovoltaic, etc. These stochastic behaviors of these sources may adversely affect the control performances since the control responses cannot track the immensely intermittent nature of such sources. Considering this uncertainty, a frequency controller that can accurately work in a small-scale power system should be designed. There are many control approaches which have been applied on the LFC process. In the literature review section, many technical papers focusing on LFC are discussed.

\subsection{Literature Review}

This section presents a load frequency controller review for both transmission and distribution systems. The survey of various controllers used for LFC is shown in Table 3.1 which categorizes the research based on the inclusion of renewable energy and electric vehicle, consideration of cyber anomalies in addition to regular disturbances and characteristics of the algorithm of adaptivity and robustness.

In Table 3.1, controllers can be categorized based on Artificial Intelligence (AI) based or classical (or analytical) control based. Contrary to the classical control methods, artificial intelligence methods are model-free and provide robust solutions for dynamical systems [56]. Since the systems we will be considering in this chapter are uncertain, several artificial intelligence approaches, including evolutionary opti- 
Table 3.1: Load Frequency Control Tabular Survey

\begin{tabular}{|c|c|c|c|c|c|c|c|c|}
\hline & 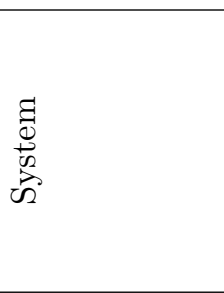 & 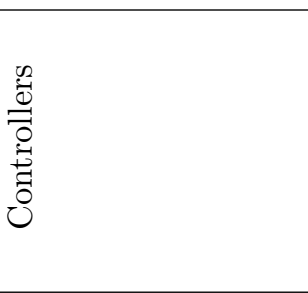 & 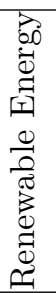 & 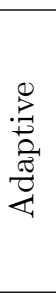 & 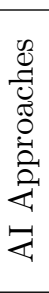 & 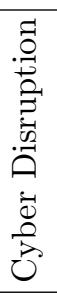 & 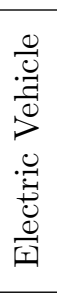 & 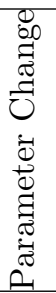 \\
\hline$[13,44]$ & Microgrid & $\begin{array}{l}\text { General Type II } \\
\text { Fuzzy Logic }\end{array}$ & * & * & * & & * & * \\
\hline $\begin{array}{l}{[45-50]} \\
{[51-56]} \\
\end{array}$ & Transmission & $\begin{array}{l}\text { Standard Fuzzy } \\
\text { controller }\end{array}$ & & & * & & & \\
\hline$[57]$ & Transmission & $\begin{array}{l}\text { Hinf \& Two-term } \\
\text { controller }\end{array}$ & & & & * & & * \\
\hline$[58-61]$ & Transmission & $\begin{array}{l}\text { Multi-objective } \\
\text { GA }\end{array}$ & & & * & & & \\
\hline $\begin{array}{c}{[62,62-65]} \\
{[66-70]}\end{array}$ & Transmission & Optimal control & & & & & & $*$ \\
\hline$[71-73]$ & Transmission & Type II fuzzy & & & $*$ & & & $*$ \\
\hline [74] & Microgrid & Emotional learning & & * & $*$ & & & $*$ \\
\hline $\begin{array}{l}{[75-79]} \\
{[80-84]}\end{array}$ & Transmission & Sliding mode & & & & & & $*$ \\
\hline$[85-90]$ & Transmission & $\begin{array}{l}\text { Evolutionary-base } \\
\text { optimization }\end{array}$ & & & & & & \\
\hline$[91]$ & Microgrid & $\begin{array}{l}\text { adaptive dynamic } \\
\text { programming }\end{array}$ & * & * & * & * & $*$ & * \\
\hline$[92]$ & Microgrid & $\begin{array}{l}\text { observer-based } \\
\text { sliding-mode }\end{array}$ & * & & & & * & * \\
\hline [93] & Microgrid & $\begin{array}{l}\text { Optimal LQR } \\
\text { Controller }\end{array}$ & & & & * & & * \\
\hline$[94]$ & Transmission & $\begin{array}{l}\text { adaptive dynamic } \\
\text { programming }\end{array}$ & & * & & * & & \\
\hline [95] & Transmission & event-triggering & & & & * & & \\
\hline$[96]$ & Microgrid & $\begin{array}{l}\text { Multivariable } \\
\text { Generalized } \\
\text { Predictive }\end{array}$ & * & & & & * & * \\
\hline$[97]$ & Microgrid & $\begin{array}{l}\text { Hebb Learning } \\
\text { Control }\end{array}$ & * & * & * & & $*$ & $*$ \\
\hline
\end{tabular}




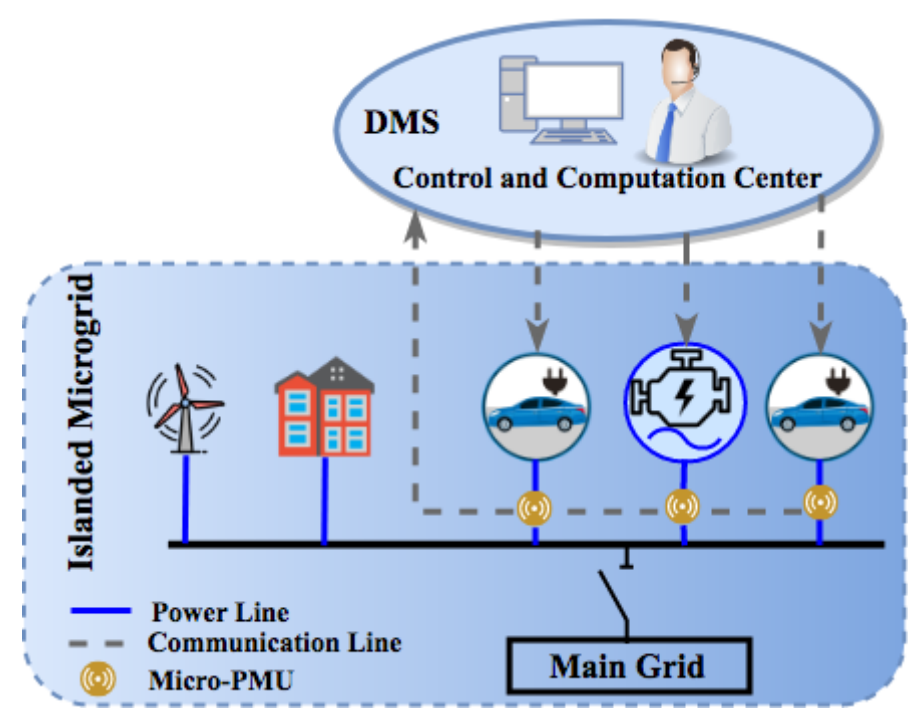

Figure 3.1: Simplified structure of the islanded microgrid

mization algorithms that are not adaptive or self-tuning are not applicable. We look at approaches inspired by human brain learning or neuroscience called Hebb Learning. Human brain emotional learning mimics how human brains make a decision based on their emotions, not logics. Hebb learning controller follows the adaptation of neurons in the brain during the learning process and not only is adaptive and self-tuning but also practical. In the rest of the chapter, Hebb learning and human brain emotional learning controllers are introduced and applied to control the frequency of microgrid. Then the system model is presented, and finally, simulation and results sections are obtained.

\subsection{Microgrid Model}

Consider an isolated microgrid, as shown in Figure 3.1, which includes a Diesel Generator (DG), a load, and two EV stations. Distribution Management System (DMS) installed at the control center supervises the microgrid and communicates with micro-Phasor Measurement Units ( $\mu \mathrm{PMUs}$ ) to receive state information. The DMS also sends logic processing and decisions from DMS to the microgrid actuators. A mathematical state-space model of microgrid and its controller is described in the following subsections [98].

\subsubsection{The State-Space Model of Microgrid}

The dynamic model of the LFC system is represented in 3.1 and expanded in (2-4) where $x(t)$ is the state variable; $u(t)$ is the control input signal; $d(t)$ is the Unknown Input (UI) which models the wind power and load perturbation; $w(t)$ and $v(t)$ are the process and measurement noises, respectively; and $y(t)$ is the measured output signal. $2 H_{t}$ denotes the equivalent inertia. The system states are shown in (3.3), where $\Delta f$ is the frequency deviation; $\Delta P_{D G}$ is the output power of Diesel Generator 
Table 3.2: The parameters of this microgrid

\begin{tabular}{|c|c|c|c|}
\hline Grid Parameters & Symbols & Values & Units \\
\hline \multirow{4}{*}{$\begin{array}{c}\text { Diesel } \\
\text { Generator }\end{array}$} & $T_{g}$ & 0.1 & $\mathrm{~s}$ \\
\cline { 2 - 4 } & $T_{d}$ & 8 & $\mathrm{~s}$ \\
\cline { 2 - 4 } & $R$ & 2.5 & $\mathrm{~Hz} / \mathrm{pu} \mathrm{MW}$ \\
\cline { 2 - 4 } & $\delta_{D G}$ & 0.001 & $\mathrm{pu} \mathrm{MW} / \mathrm{s}$ \\
\cline { 2 - 4 } & $\mu_{D G}$ & 0.04 & $\mathrm{pu} \mathrm{MW}$ \\
\hline \multirow{4}{*}{$\begin{array}{c}\text { EV } \\
\text { Station 1 }\end{array}$} & $T_{e 1}$ & 1 & $\mathrm{~s}$ \\
\cline { 2 - 4 } & $\delta_{e 1}$ & 0.01 & $\mathrm{pu} \mathrm{MW} / \mathrm{s}$ \\
\cline { 2 - 4 } & $\mu_{e 1}$ & 0.025 & $\mathrm{pu} \mathrm{MW}$ \\
\cline { 2 - 4 } & $E_{\max }$ & 0.95 & $\mathrm{pu} \mathrm{MWh}$ \\
\cline { 2 - 4 } & $E_{\min }$ & 0.80 & $\mathrm{pu} \mathrm{MWH}$ \\
\hline \multirow{4}{*}{$\begin{array}{c}\text { EV } \\
\text { Station 2 }\end{array}$} & $T_{e 2}$ & 1 & $\mathrm{~s}$ \\
\cline { 2 - 4 } & $\delta_{e 2}$ & 0.01 & $\mathrm{pu} \mathrm{MW} / \mathrm{s}$ \\
\cline { 2 - 4 } & $\mu_{e 2}$ & 0.016 & $\mathrm{pu} \mathrm{MW}$ \\
\cline { 2 - 4 } & $E_{\max }$ & 0.90 & $\mathrm{pu} \mathrm{MWh}$ \\
\cline { 2 - 4 } & $E_{\min }$ & 0.80 & $\mathrm{pu} \mathrm{MWH}$ \\
\hline \multirow{2}{*}{ Grid Inertia } & $H_{t}$ & 7.11 & \multicolumn{1}{c}{$\mathrm{s}$} \\
\hline
\end{tabular}

(DG), $\Delta X_{G}$ is the incremental valve position of governor, and $\Delta P_{E 1}$ and $\Delta P_{E 2}$ are the output powers of the first and second EV stations [98]. The relevant microgrid parameters are provided in Table 3.2.

$$
\begin{aligned}
& \left\{\begin{array}{l}
\dot{x}(t)=A_{c} x(t)+B_{c} u(t)+D d(t) \\
y(t)=C x(t)
\end{array}\right. \\
& A_{c}=\left[\begin{array}{ccccc}
0 & \frac{1}{2 H_{t}} & 0 & \frac{1}{2 H_{t}} & \frac{1}{2 H_{t}} \\
0 & \frac{-1}{2 T_{d}} & \frac{1}{T_{d}} & 0 & 0 \\
\frac{-1}{R T_{g}} & 0 & \frac{-1}{T_{g}} & 0 & 0 \\
0 & 0 & 0 & \frac{-1}{T_{e 1}} & 0 \\
0 & 0 & 0 & 0 & \frac{-1}{T_{e 2}}
\end{array}\right] \\
& B_{c}=\left[\begin{array}{ccc}
0 & 0 & 0 \\
0 & 0 & 0 \\
\frac{1}{T_{g}} & 0 & 0 \\
0 & \frac{1}{T_{e 1}} & 0 \\
0 & 0 & \frac{1}{T_{e 2}}
\end{array}\right] \\
& C=\left[\begin{array}{lllll}
1 & 0 & 0 & 0 & 0
\end{array}\right] \\
& D=\left[-\frac{1}{2 H_{t}} \quad 0 \quad 00 \quad 0 \quad 0\right] \\
& x(t)=\left[\begin{array}{lllll}
\Delta f & \Delta P_{D G} & \Delta X_{G} & \Delta P_{E 1} & \Delta P_{E 2}
\end{array}\right]^{T} \\
& u=\left[\begin{array}{lll}
\Delta u_{D G} & \Delta u_{E 1} & \Delta u_{E 2}
\end{array}\right]^{T}
\end{aligned}
$$

The microgrid model considers an equivalent EV dynamic model and diesel gen- 


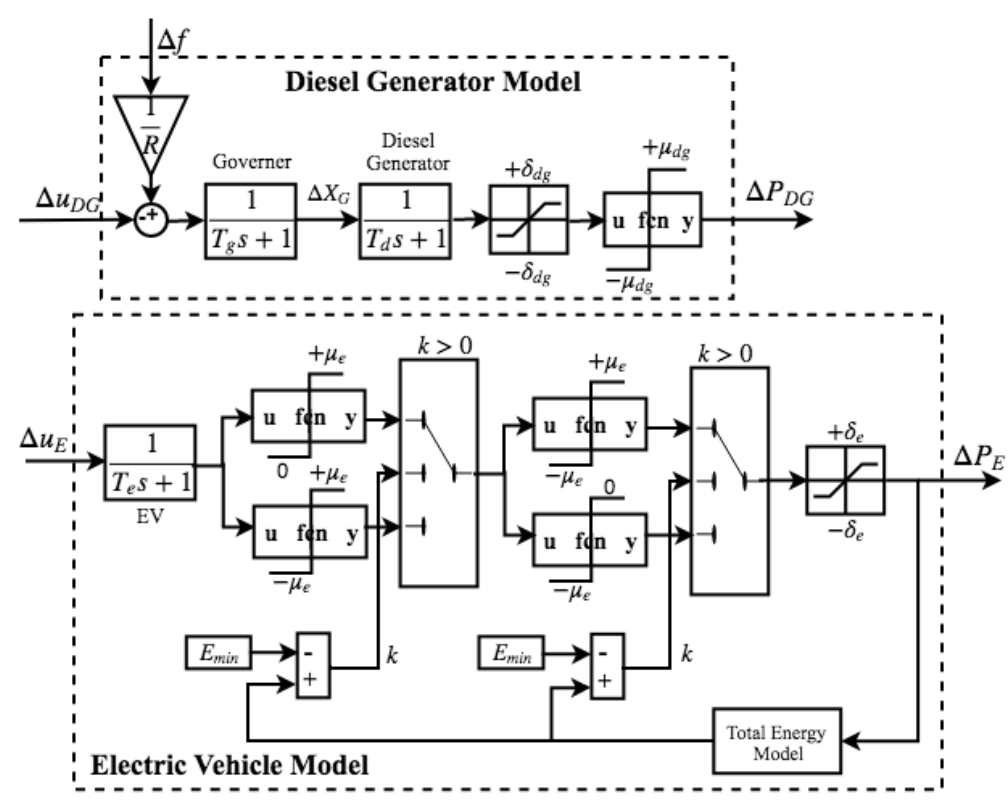

Figure 3.2: The diesel generator and electric vehicle model in LFC of the microgrid

erator equivalent model, as shown in Figure 3.2. Here $T_{e}$ denotes time constant, and $\Delta u_{E}$ denotes the control inputs of the EVs with consideration of mechanical limits of the power ramp rate $\delta_{e}$, power increment $\mu_{e}$ and maximum $E_{\max }$ and minimum $E_{\min }$ controllable energy [98]. The control input $\Delta u_{D G}$ adjusts the output power of the DG $\Delta P_{D G}$. The mechanical characteristics of the DG are given by speed regulation coefficient $R$, the time constant $T_{d}$ of DG and the time constant of the governor $T_{g}$, and limits of the power ramp rate $\delta_{d g}$ and the power increment $\mu_{d g}$ [99].

\subsubsection{The Microgrid Control Structure}

The input to LFC controller in Figure 3.3 is frequency deviation $\Delta f$, and its outputs serve as control input $\Delta u_{D G}$ to DG and as control inputs $\Delta u_{E 1}$ and $\Delta u_{E 2}$ to both EV stations. As illustrated in Figure 3.3, two control responses, primary and secondary, are considered to mitigate the frequency deviation. The primary control is coordinated with the governor of diesel-driven synchronous generator $\Delta X_{G}$ to alter the mechanical settings and accordingly adjust the generated power of DG. The primary control does not have a dependency on the communication network and measuring components [100]. The secondary control in Figure 3.3 is designed so that the frequency is driven to its nominal value by fine tuning using control commands $\Delta u_{E 1}$ and $\Delta u_{E 2}$ to actuate EVs to inject or absorb restorative power. In the next sections, two artificial intelligence control strategies are proposed to control the microgrid frequency. 


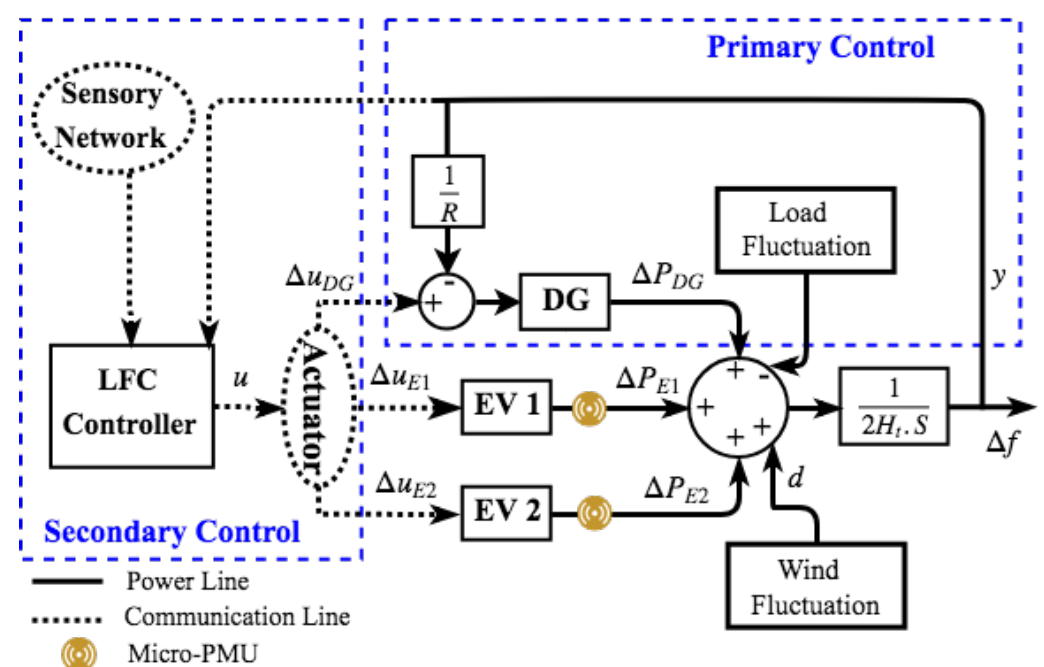

Figure 3.3: Diagram of Load Frequency Control

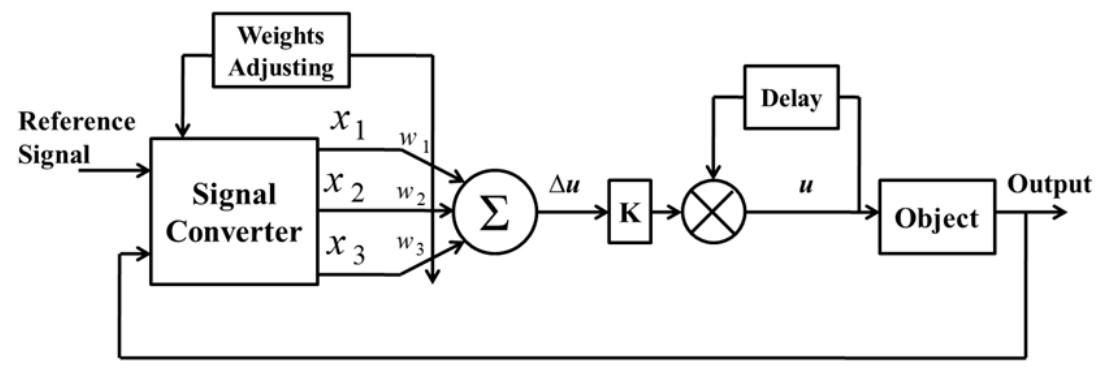

Figure 3.4: Diagram of a single neuron PID control system based on the Hebb learning algorithm [101]

\subsection{Hebb Learning Based Controller}

Figure 3.4 shows the single neuron PID controller based on Hebbian learning with proportional, integral and differential errors forming the weighted input $X=\left\{x_{1}(k)\right.$, $\left.x_{2}(k), x_{3}(k)\right\}$ at time instant $k$ [101]. The weights shown as $W_{i}$ in (3.5) must be normalized to avoid their infinite growth.

$$
W_{i}^{\prime}(k)=W_{i}(k) / \sum_{i=1}^{3}\left|W_{i}(k)\right|
$$

The output of the controller is as shown in (3.6), and the input vector is the weighted sum of the errors as shown in (3.6) where $e$ is an error due to deviation in frequency from the desired frequency. $K$ is proportionality constant. Since the input to the neuron is the weighted sum of errors the weights have to be carefully 
chosen and hence the next section discusses learning methods to select weights.

$$
\begin{gathered}
u(k)=u(k-1)+K \sum_{i=1}^{3} W_{i}^{\prime}(k) x_{i}(k) \\
\left\{\begin{array}{c}
x_{1}=e(k)-e(k-1) \\
x_{2}=e(k) \\
x_{3}=e(k)-2 e(k-1)+e(k-2)
\end{array}\right.
\end{gathered}
$$

\subsubsection{Learning Methods for Weights}

There are many weights-learning rules for training neural networks four of which are discussed in this chapter. These learning rules tune the varying weights of Proportional, Integral, and Derivative (PID) coefficients. In this manner, a selftuning PID controller is adopted for LFC of this microgrid. These four learning rules are mentioned in (3.8-3.11) [97]:

Non-supervised Hebb learning rule: The controller output and the corresponding PID signals error are multiplied in this learning rule:

$$
\left\{\begin{array}{l}
W_{1}(k)=W_{1}(k-1)+\eta_{P} x_{1}(k-1) u(k-1) \\
W_{2}(k)=W_{2}(k-1)+\eta_{I} x_{2}(k-1) u(k-1) \\
W_{3}(k)=W_{3}(k-1)+\eta_{D} x_{3}(k-1) u(k-1)
\end{array}\right.
$$

Supervised Delta learning rule: The controller output and the error between the reference and actual frequency e are multiplied in this learning rule:

$$
\left\{\begin{array}{l}
W_{1}(k)=W_{1}(k-1)+\eta_{P} e(k-1) u(k-1) \\
W_{2}(k)=W_{2}(k-1)+\eta_{I} e(k-1) u(k-1) \\
W_{3}(k)=W_{3}(k-1)+\eta_{D} e(k-1) u(k-1)
\end{array}\right.
$$

Supervised Hebb learning: The controller output, the error between the reference and actual frequency $e$, and the corresponding PID error are multiplied in this learning rule:

$$
\left\{\begin{array}{c}
W_{1}(k)=W_{1}(k-1)+\eta_{P} e(k-1) x_{1}(k-1) u(k-1) \\
W_{2}(k)=W_{2}(k-1)+\eta_{I} e(k-1) x_{2}(k-1) u(k-1) \\
W_{3}(k)=W_{3}(k-1)+\eta_{D} e(k-1) x_{3}(k-1) u(k-1)
\end{array}\right.
$$

Improved Hebb learning: The controller output, the error between the reference and actual frequency e and another term, which is the summation of error and error difference, are multiplied in this learning rule:

$$
\left\{\begin{array}{l}
W_{1}(k)=W_{1}(k-1)+\eta_{P} e(k-1) u(k-1)[e(k)+\Delta e(k)] \\
W_{2}(k)=W_{2}(k-1)+\eta_{I} e(k-1) u(k-1)[e(k)+\Delta e(k)] \\
W_{3}(k)=W_{3}(k-1)+\eta_{D} e(k-1) u(k-1)[e(k)+\Delta e(k)]
\end{array}\right.
$$

where $\eta_{P}, \eta_{I}$ and $\eta_{D}$ are coefficients for proportional integral and differential learning speed, respectively. In all these learning rules, these coefficients are tuned based on 


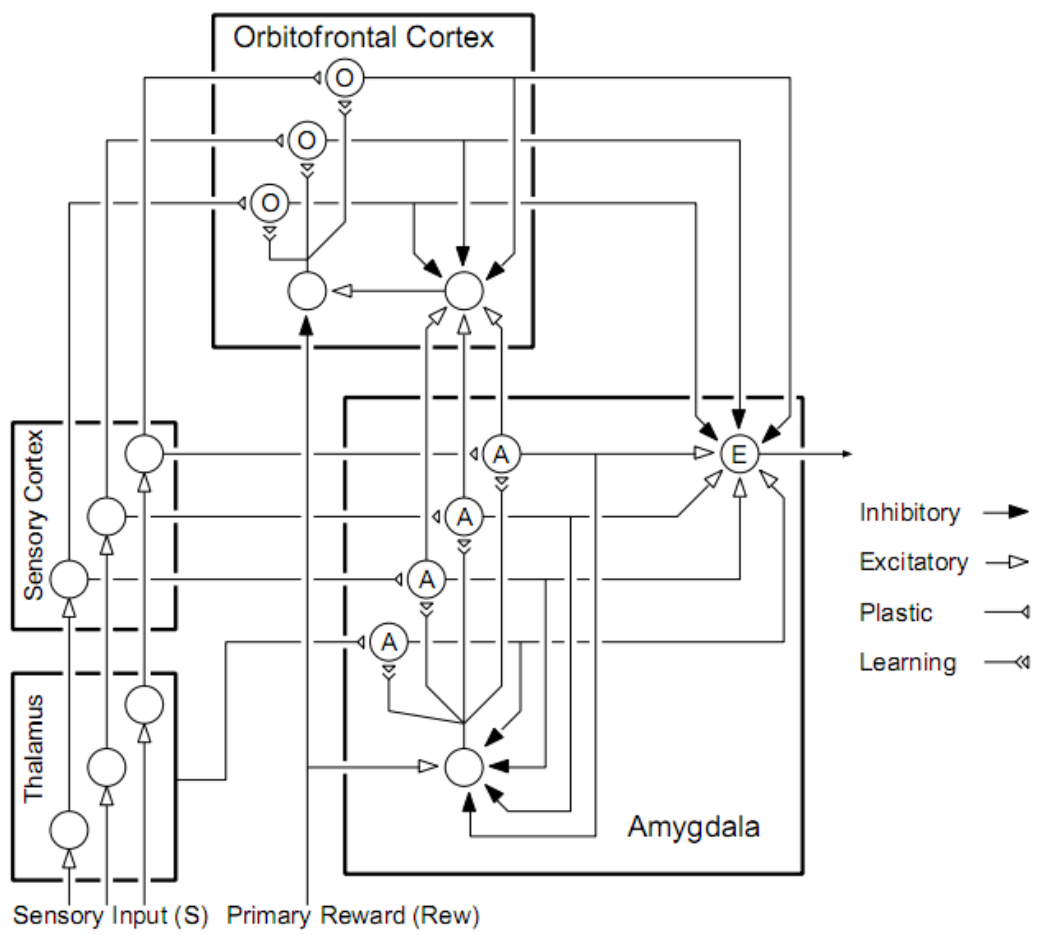

Figure 3.5: Detailed diagram of emotional learning system of the human brain [102]

their influences on the control performance. In all control processes, frequency error and its derivatives are very important. The frequency error has a higher priority than its first-order derivative and the first-order derivative have higher priority than the second-order derivative in this control mechanism. Supervised Hebb learning and Improved Hebb learning are applied to LFC in this chapter.

\subsection{Human Brain Emotional Learning Controller}

Moren and Balkenious introduced this controller for the very first time in 2000. These researchers tried to develop computational models for those parts of human brains which perform emotional decision making. In [102], a new model was introduced to carry out brain emotional processing. This model includes Amygdala, orbitofrontal cortex, thalamus, and finally, sensory cortex illustrated in Figure 3.5 and Figure 3.6. Considering this model, the Amygdala-orbitofrontal system performs the learning process in two steps. First, the input stimulant signals are assessed, and in the next step, this evaluation is used as intensifying coefficients in response to the stimulant. Amygdala is one of the main structures in brains which exists in an almost uniform way in large scale structures among different species of animals. Amygdala, which is one of the primary parts in brains, is a small part of temporal lobe which performs the emotional assessment of stimulant. These assessments are applied in emotional states and reactions, attention signals, also in long-term memory. Some of the intrinsic stimulants, including pain, hunger and some smells etc., can excite the Amygdala. The Amygdala's response to these stimulants is ap- 


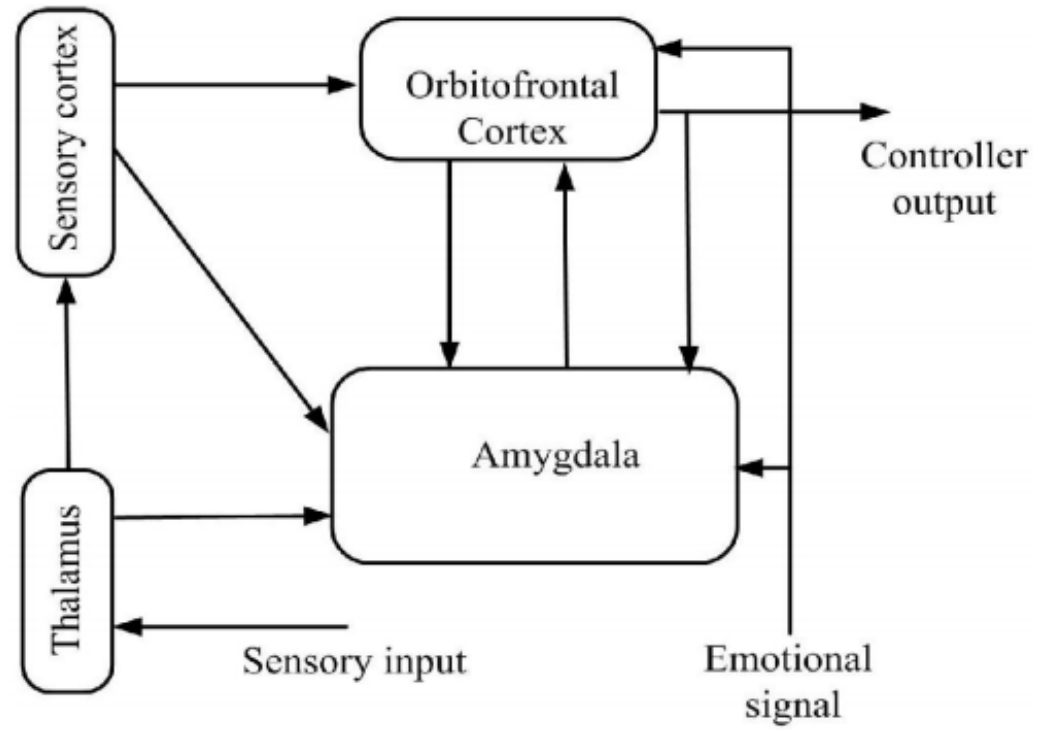

Figure 3.6: Diagram of the proposed computational model of the human brain learning mechanism

plied in learning process. The scientist's investigations show that the animals which are suffering from damaged Amygdala have difficulties in learning. Therefore, this point proves that learning occurs in Amygdala. However, the orbitofrontal cortex is used to modify unsuitable responses of Amygdala. A large number of experiments of patients with impaired orbitofrontal-cortex have shown that they cannot adapt themselves to new conditions. Ignoring the details, the diagram of the brain emotional learning system is illustrated in Figure 3.6. In following, this will be used to describe the proposed computational model for Amygdala-orbitofrontal system. The computational model output MO (the response of Amygdala-orbitofrontal structure to input stimulant SI) is equal to [103]:

$$
M O=A O-O C O
$$

where $A O$ and $O C O$ are the Amygdala and orbitofrontal outputs respectively and their formula are obtained in (3.13):

$$
\begin{aligned}
& A O=G_{a} \quad S I \\
& O C O=G_{o c} \quad S I
\end{aligned}
$$

Where, $G_{a}$ and $G_{o c}$ are the corresponding gains of Amygdala and orbitofrontal parts, respectively. Learning law in orbitofrontal and Amygdala parts is demonstrated in Equation (3.14).

$$
\begin{aligned}
& \Delta G_{a}=k_{1} \cdot \max (0, E C-A O) \geq 0 \\
& \Delta G_{o c}=k_{2} \cdot(M O-E C)
\end{aligned}
$$

Where, $K_{1}, K_{2}$ are the learning rates for Amygdala and orbitofrontal parts, 


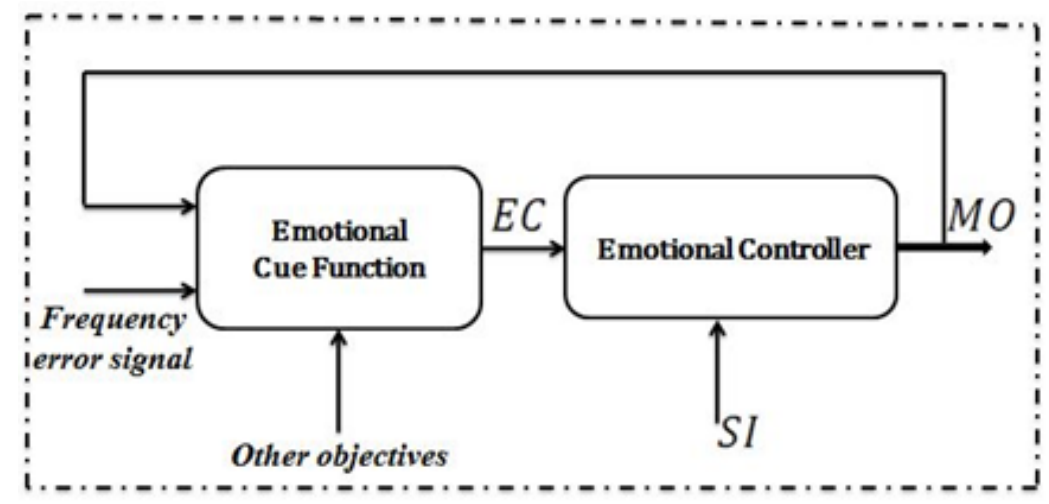

Figure 3.7: Diagram of Emotional Learning controller implemented in LFC

respectively. Considering (3.14), because of using the max operator, the gain of Amygdala part is always monotonously increasing variation, and this is confirmed with physiological evidence that the Amygdala is unable to forget what it has already learned.

\subsubsection{LFC Intelligent Control Based on Emotional Learning and its Bi-Objective Control Structure}

By combining Equations (3.13) to (3.14), (3.15), we obtain:

$$
M O=\left(G_{a}-G_{o c}\right) \cdot S I \equiv G(S I, E C, \ldots) \cdot S I
$$

The output of Amygdala-orbitofrontal system is equal to a varying gain $G$, which depends on many factors, including emotional signal $E C$, input stimulant SI, etc., multiplied by input stimulant $S I$ [104]. A proper and simple idea to express stimulant input $S I$ is the familiar controller of PID:

$$
S I=k_{P} \cdot e_{f}+K_{I} \cdot \int_{0}^{t} e_{f} d t+K_{D} \cdot \dot{e}_{f}
$$

where $e_{f}=f_{\text {ref }}-f$ is the frequency error of the closed-loop system. The emotional signal $E C$ show how properly the LFC controller is working. Thus, EC could be defined as a weighted combination of objective functions and can be described as (3.17).

$$
E C=a_{e c 1} \cdot e_{f}+a_{e c 2} \cdot M O+a_{e c 3} \cdot(\text { Other Control Objective })
$$

In this equation $a_{e c 1}$, aec 2, aec 3 are the weight coefficients of frequency error, computational model error and other control objective, respectively. In the equation, frequency deviation signal $e_{f}$ is the deference between reference and real frequency signal. Computational model error is named control effort feedback signal that leads to better performance than many controllers. The proposed LFC control strategy is presented in Figure 3.7. In the next section, two AI based controllers are utilized 


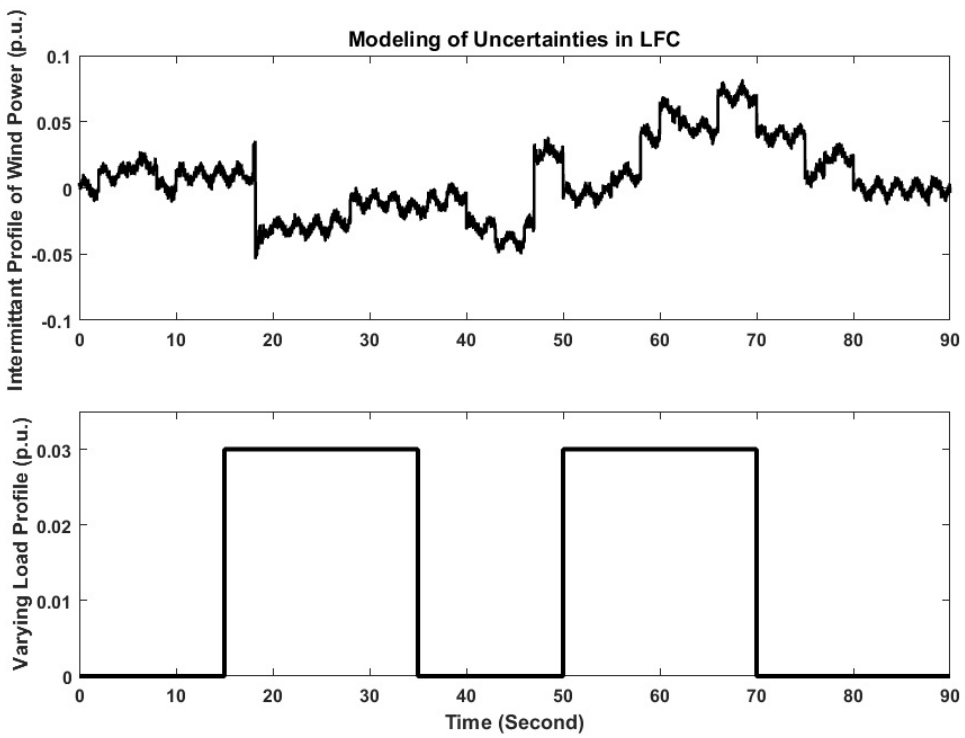

Figure 3.8: Load and Wind Power Profiles

for LFC of the Microgrid discussed earlier.

\subsection{Simulation and Results}

In this section, LFC results of the microgrid are shown using the proposed and standard PID controllers. The results are discussed in four different scenarios to noticeably compare control response of the proposed controllers and PID controller. Figure 3.8 demonstrates wind power fluctuation and load profiles. A real data of wind fluctuation is considered to model the stochastic behavior of wind power, which is obtained from an offshore wind farm in Sweden [105]. This collected data is zeromean profile during a period of 90 seconds. Both EV sources are modeled identical and with the same parameters. As mentioned earlier, just two of the discussed types of Hebb learning rules, Supervised Hebb learning, and Improved Hebb learning, are applied to control the frequency of this system. These control approaches are adopted to control the frequency of microgrid in Figure 3.3. They manage and control the power of sources (output power from DG and EVs) to supply the load in the presence of very intermittent behaviors of wind power and load profiles in different scenarios. Contrary to the standard PID controller, thanks to the adaptive and self-tuning mechanism, the proposed controllers can control the frequency even in very fluctuating effects of wind power energy.

\subsubsection{Scenario 1}

This scenario shows the effects of the load profile on LFC without wind and EVs. Frequency responses for all controllers, Emotional Learning controller, Hebb learning controller with Supervised Hebb learning and Improved Hebb learning rules and 


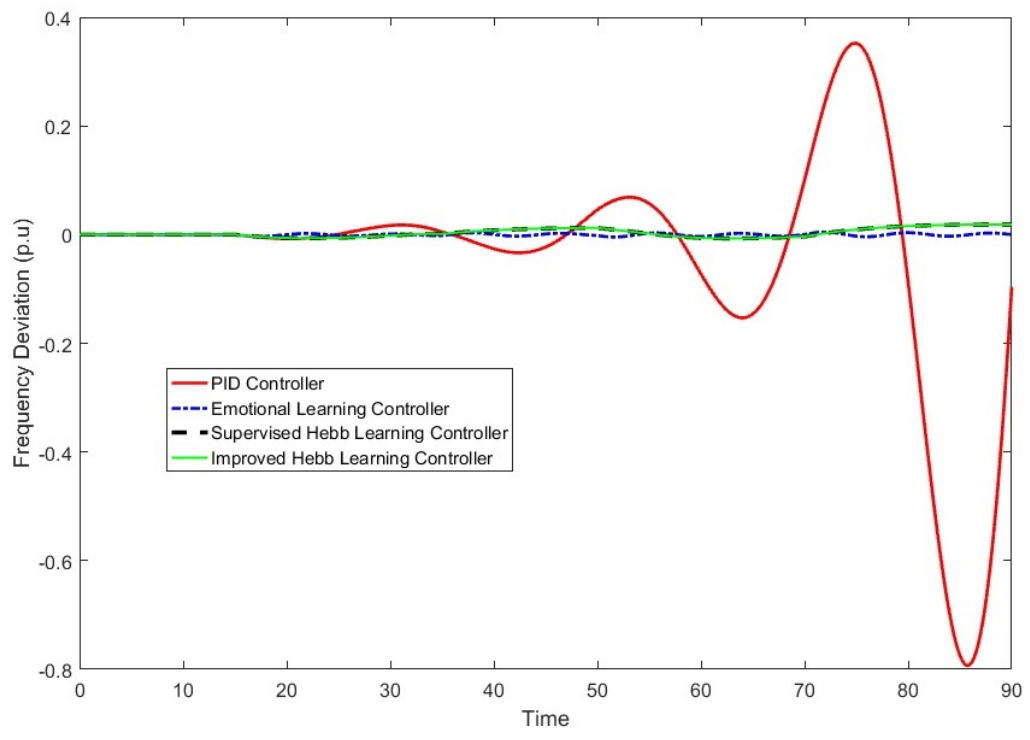

Figure 3.9: Frequency deviation in Scenario 1

standard PID controller, are depicted in Figure 3.9, and power output of DG is revealed in Figure 3.10. It is clear that in spite of the PID controller, which is not able to appropriately provide power from DG source (the only power source in this scenario) to control frequency, Emotional Learning and both Hebb learning controllers can control the frequency under this scenario.

\subsubsection{Scenario 2}

The conditions are identical to Scenario 1; but in this scenario, EV sources operate in V2G mode are included to supply the demand. Frequency responses for all controllers, Emotional Learning controller, Hebb learning controller with Supervised Hebb learning, Improved Hebb learning, and PID controller, are depicted in Figure 3.11 and DG and EV power products are demonstrated in Figure 3.12. It is observed that Emotional and Hebb Learning controllers have better control performance than the PID controller.

\subsubsection{Scenario 3}

The conditions are identical to Scenario 2; but in this scenario, wind power fluctuation is included. Frequency responses for all controllers, Emotional Learning controller, Hebb learning controller with Supervised Hebb learning, Improved Hebb learning and, the PID controller, are seen in Figure 3.13 and DG and EV power products are demonstrated in Figure 3.14. It is observed that although wind power profile brings very high fluctuations in this scenario, the Emotional and Hebb Learning controllers can properly control the frequency. 


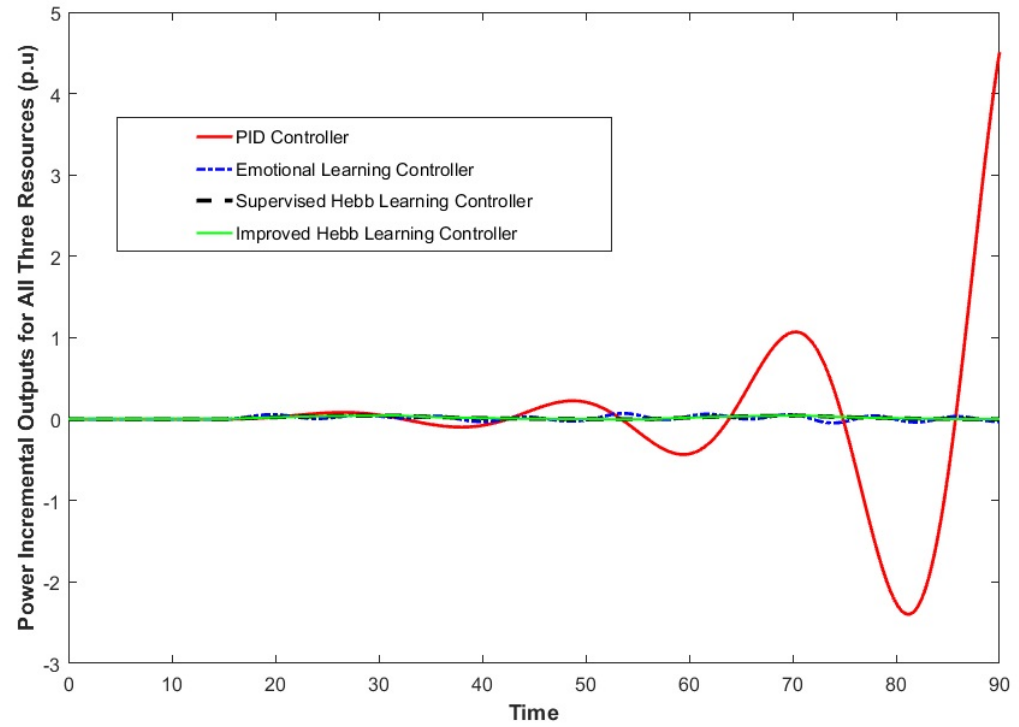

Figure 3.10: Power incremental output for Scenario 1

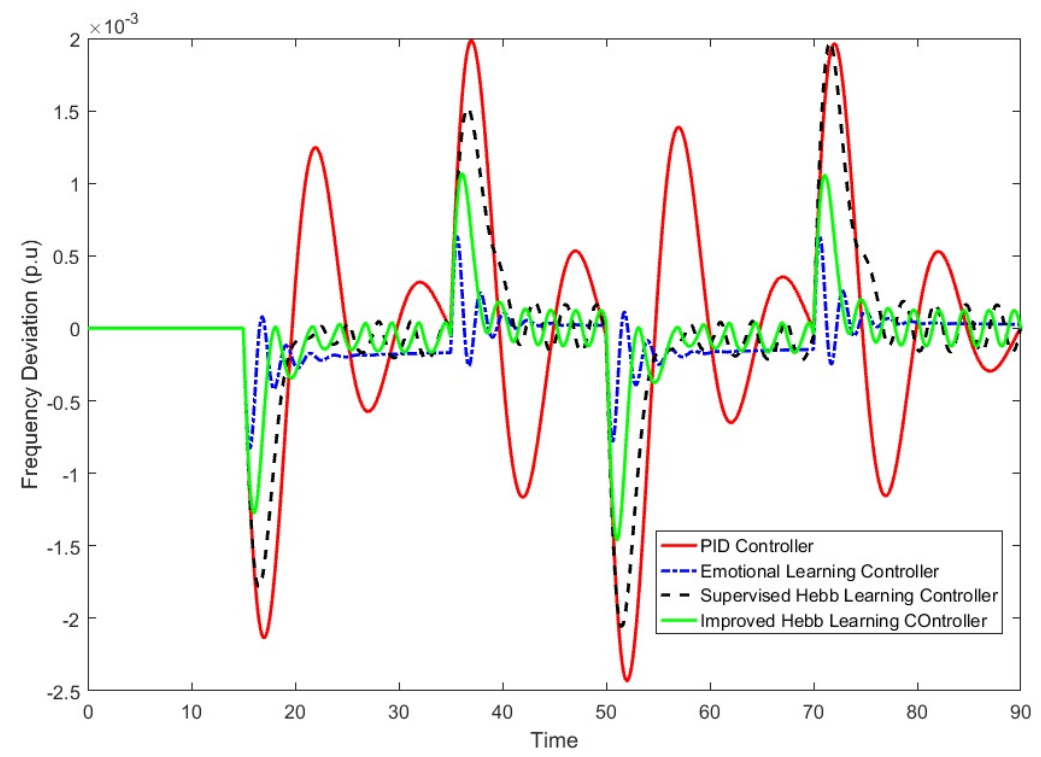

Figure 3.11: Frequency deviation in Scenario 2 

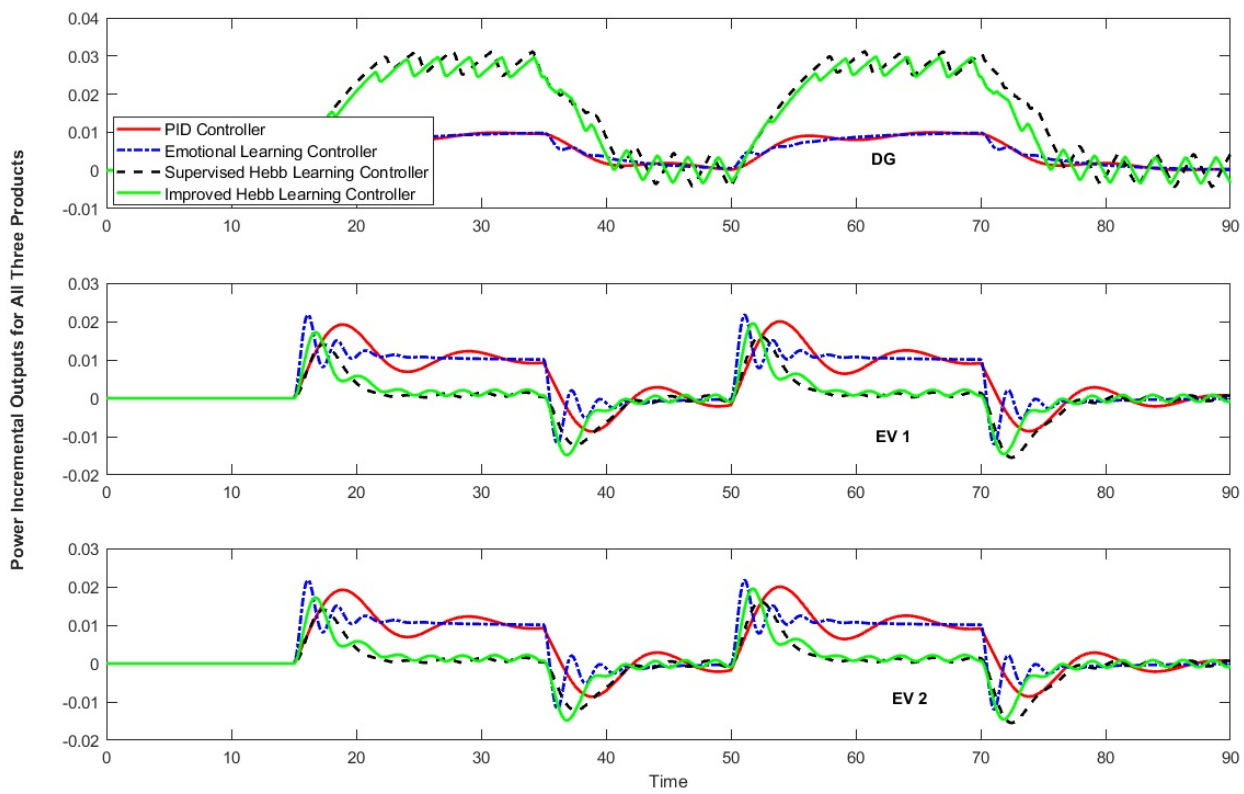

Figure 3.12: Power incremental output for Scenario 2

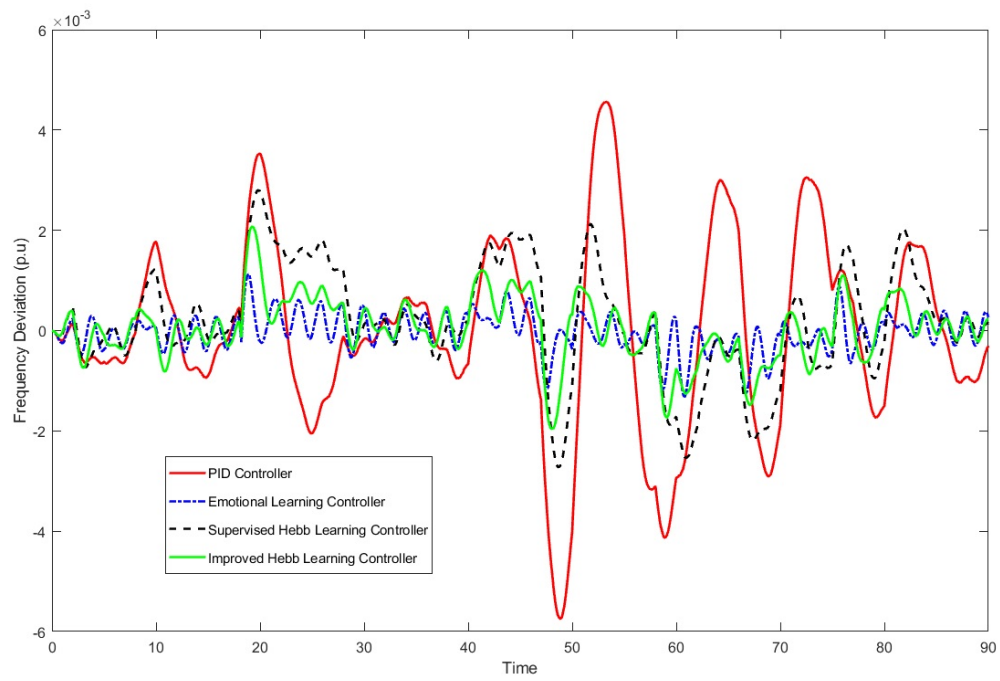

Figure 3.13: Frequency deviation in Scenario 3 


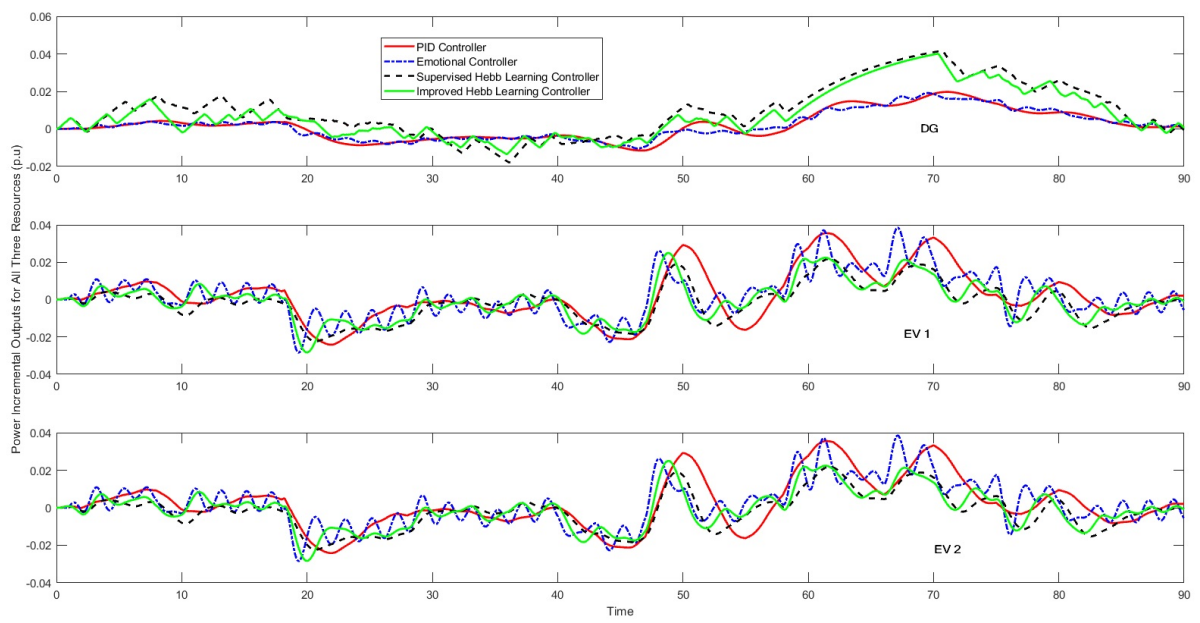

Figure 3.14: Power incremental output for Scenario 3

\subsubsection{Scenario 4}

The conditions are identical to Scenario 3; but in this scenario, varying load profile is included. Frequency responses for all controllers, Emotional Learning controller and Hebb learning controller with Supervised Hebb learning, Improved Hebb learning, and the PID controller, are depicted in Figure 3.15 and DG and EV power products are demonstrated in Figure 3.16. It is observed that Emotional Learning and both learning trends of Hebb controllers have considerably better control responses than PID controller.

In order to present a quantitative comparison among controllers in different cases, the Integral of Time multiplied by the Absolute value of Error ITAE is applied. This criterion can consider overshoot, steady-state error, and settling time [97].

$$
I T A E=\int_{0}^{T} t\left|e_{f}\right| d t
$$

In Table 3.3, the controllers' performances are quantitatively compared using the ITAE index. It is found that, in all scenarios, Improved Hebb learning controller has better control performance than Supervised Hebb learning controller. Since the frequency error signal is involved in the learning rule of improved Hebb learning controller, its performance is more effective than the performance of Supervised Hebb learning controller. Also, Supervised Hebb learning controller works significantly better than PID controller because, in spite of PID controller, Supervised Hebb learning controller has an adaptive control structure. Also, owing to the high ability of learning and adaptivity, Emotional Learning controller works considerably better than all other controllers discussed in this chapter. It is seen from Table 3.3 that due to the self-tuning structure, Neuroscience based controllers carry out their control function far superior to the standard PID controller. Without effects 


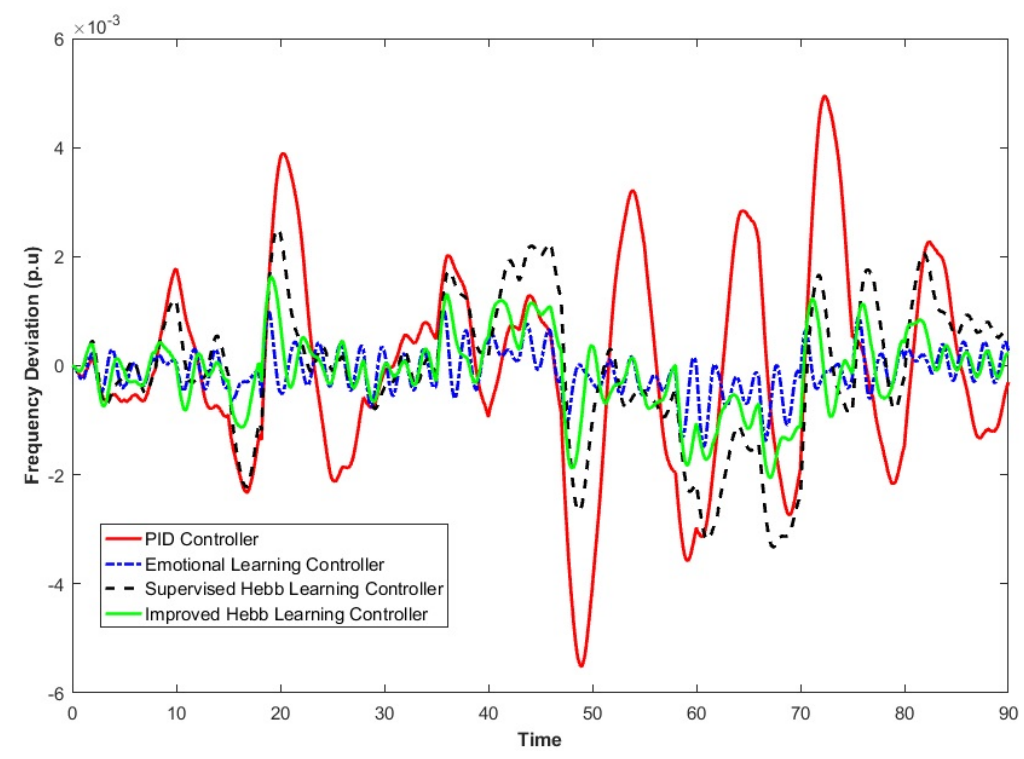

Figure 3.15: Frequency deviation in Scenario 4

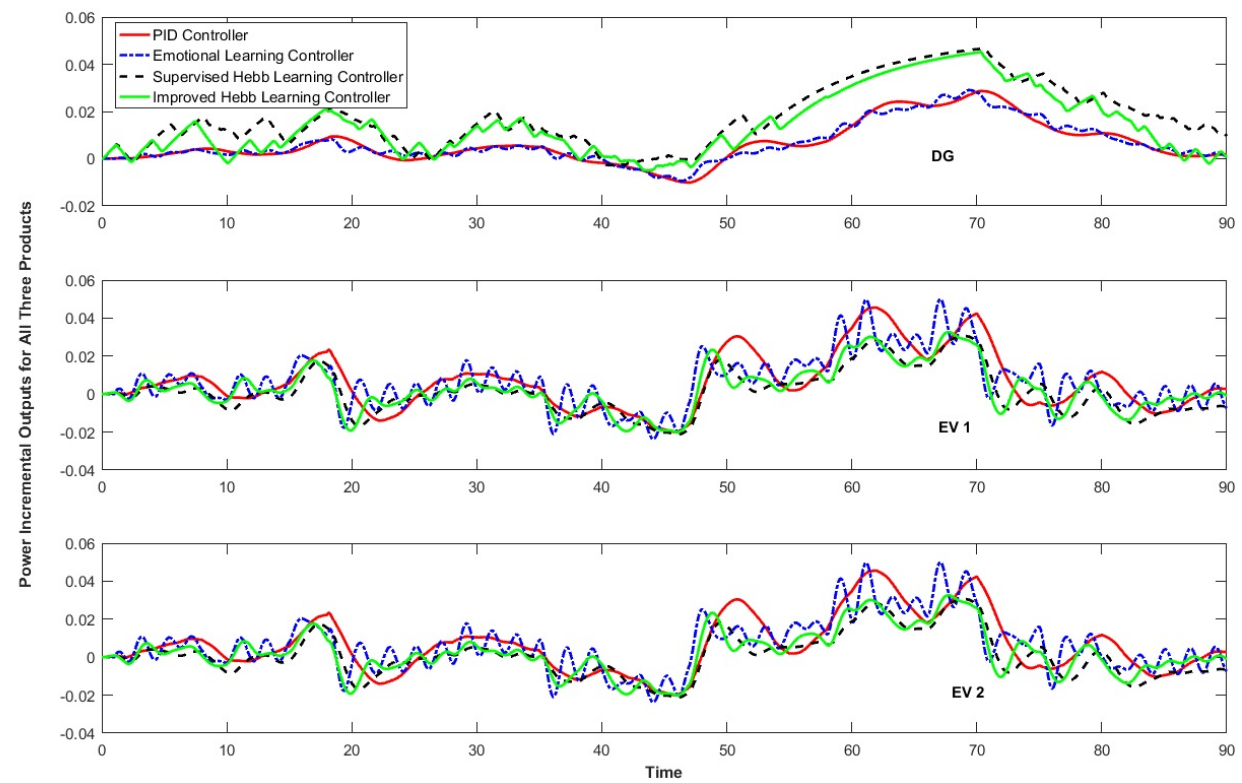

Figure 3.16: Power incremental output for Scenario 4 
Table 3.3: ITAE Index Evaluation For All Controllers In All Four Scenarios

\begin{tabular}{|l|l|l|l|l|}
\cline { 2 - 5 } \multicolumn{1}{c|}{} & Scenario 1 & Scenario 2 & Scenario 3 & Scenario 4 \\
\hline PID & $7.4135 \mathrm{e}+04$ & 251.1042 & 647.9046 & 678.2314 \\
\hline $\begin{array}{l}\text { Supervised } \\
\text { Hebb Learning }\end{array}$ & $3.5980 \mathrm{e}+03$ & 129.5125 & 367.2134 & 472.1358 \\
\hline $\begin{array}{l}\text { Improved } \\
\text { Hebb Learning }\end{array}$ & $3.6139 \mathrm{e}+03$ & 65.9227 & 199.1428 & 244.7095 \\
\hline $\begin{array}{l}\text { Emotional } \\
\text { Learning }\end{array}$ & 788.6041 & 47.9627 & 118.4581 & 134.2963 \\
\hline
\end{tabular}

of wind fluctuation and EV sources, the frequency is controlled within limits and in case we have large deviations in load and wind power, Neuroscience based controllers restrict the frequency deviation. It is easy to see that Emotional Learning controller outperforms all other controllers, including both Hebb learning methods and standard PID controller.

\subsection{Conclusion}

Examining the simulation and results, we conclude that the proposed artificial intelligence methods have significantly better control responses than a standard PID controller. In this chapter, some real features in power grids, such as the stochastic behavior of wind energy and load profile, are considered. Since this system is very dynamic, and it is not simple to be accurately modeled, leveraging artificial intelligence based control methodologies are very interpretable. These control approaches do not need the accurate mathematic model of the system in their control procedure which is their main advantage. 


\section{Chapter 4}

\section{Resilient Control of Microgrids}

\subsection{Introduction}

The interconnection of distributed generations (DGs), energy storage, Electric Vehicles (EV), loads, and communication structure results in smaller grid-like structures called microgrids. Microgrids have been increasingly deployed at educational campuses, hospitals, military sites and have been greatly utilized in remote applications such as telecom, rural households, etc. Compared to legacy distribution grids, microgrids highly rely on control configuration that involves coordination, monitoring, and integration that, in turn, leads to the more efficient, reliable and stable operation than standard distribution grids [4]. Microgrids employ Advanced Metering Infrastructure (AMI) for data collection, and hence, information exchange occurs through communication networks creating a new paradigm of energy systems called Cyber-Physical Microgrids (CPM). Since modern microgrids locally harness renewable energy sources (RES) they result in fewer power losses with the added advantage of decreased environmental pollution, unlike legacy power systems. Normally dynamic control strategy is utilized for microgrids, including hierarchical control, primary control (droop control), and secondary control. The primary control response is the immediate adjustment of power output by the governor or electronic controller to address any changes in the microgrid frequency or speed of rotating energy sources. Intermittent and low-inertia characteristics of RESs along with uncertainties in loads, can degrade the primary control of microgrid. This degradation creates an imbalance between supply and consumption leading to frequency deviations and even frequency instability which can be catastrophic for islanded microgrids since there is no frequency and voltage support from the main grid.

Additionally, to support the primary control response, a secondary control unit can be utilized specially for energy sources like batteries and electric vehicles that do not have a governor-based or droop-based control. Secondary control of microgrids is a supervisory control utilizing measurements and communication systems. Utilizing battery as storage for the control is not economically efficient hence electric vehicles in vehicle-to-grid ( $\mathrm{V} 2 \mathrm{G})$ mode have been considered here especially due to their almost $90 \%$ availability during the day and low power loss ratio. $[4,7,8,15]$. 
Normally wireless and/or mobile communication networks are utilized for secondary control and communication of microgrids and IEEE 802.15.4x (e.g., ZigBee) or IEEE 802.11.x, a more secure protocol is employed. These protocols compromise security and enable manipulation of data by adversaries [106]- [107]. One of these cyber disruptions is Denial of Service (DoS), [108] which occurs when smart grid's components are not available. Another cyber disruption is the Time Delay Switch (TDS) or Signal Delay Attack, which occurs when a delay is introduced in communication channels $[109,110]$. Another severe cyber disruption is False Data Injection (FDI) that occurs when information is intercepted or manipulated [111] through actuators or sensors. FDI agents compromise the exchange of real-time data among $\mu$ PMUs measurements, control center, and energy sources that may lead to frequency deviation and finally trigger negative defensive protection reactions, such as shedding loads or disconnecting generators [112]. The unplanned defensive protection reactions can cause permanent equipment impairment and cascading outages leading to blackouts. This, in turn, causes adverse social impact and economic losses asserting the importance of [112] resilient control, crucial for sustaining normal operations of CPM. The 2015 Ukraine Blackout events have indicated the occurrence of FDI disruptions realizing their practical occurrence and the devastating effects [113]. The identification of FDI in power grids using a reinforcement learning method has been discussed in [114], whereas $[115,116]$ use extreme learning machine (ELM) techniques. These techniques can detect FDI; however, the state estimation is static and not dynamic in [114]. Prediction method based on the spatial correlation of data in $[115,116]$ is dependent on a pre-defined threshold. [117] eliminates the dependency on the threshold by using a Gaussian-Mixture detection scheme to detect stealthy manipulations and clustering. This method has a high dependency on historical adversarial data for training the model, which is difficult to obtain and moreover, large scale implementations would be challenging. Another group of researchers has utilized state estimators and observers for attack identification in smart grids [118], as not all system states can be measured due to cost [119-121]. These approaches seem to be reasonable and do not suffer the curse of dimensionality. Additionally, due to the uncertainties emerging from renewable energies and disruptions from FDIs, the measurements and system states are continually perturbed; hence, they cannot be directly utilized for feedback control. Kalman Filters have been widely utilized for state-estimations; however, their performance accuracy is restricted by a pre-defined threshold $[118,122]$. Traditional Leuenberger observers do not distinguish between signals and consider signals with uncertainty as well [123]. Sliding mode observers can be utilized however they suffer from high chattering $[124,125]$. The distinction of uncertainties, elimination of exogenous disruptions, and chattering can be eliminated by Unknown Input Observers (UIO) [123]. [126,127] propose model-based estimation and detection of unknown input and do not address controlling and thwarting of the disruptions. In this section, an optimal UIO based controller is developed for an islanded microgrid which is not dependent on the predefined threshold and also can detect both uncertainties and FDI and mitigate them accordingly. These uncertainties and FDI are formulated as unknown inputs. The cyber disruption considered here is an intelligent stealthy FDI which affects control 


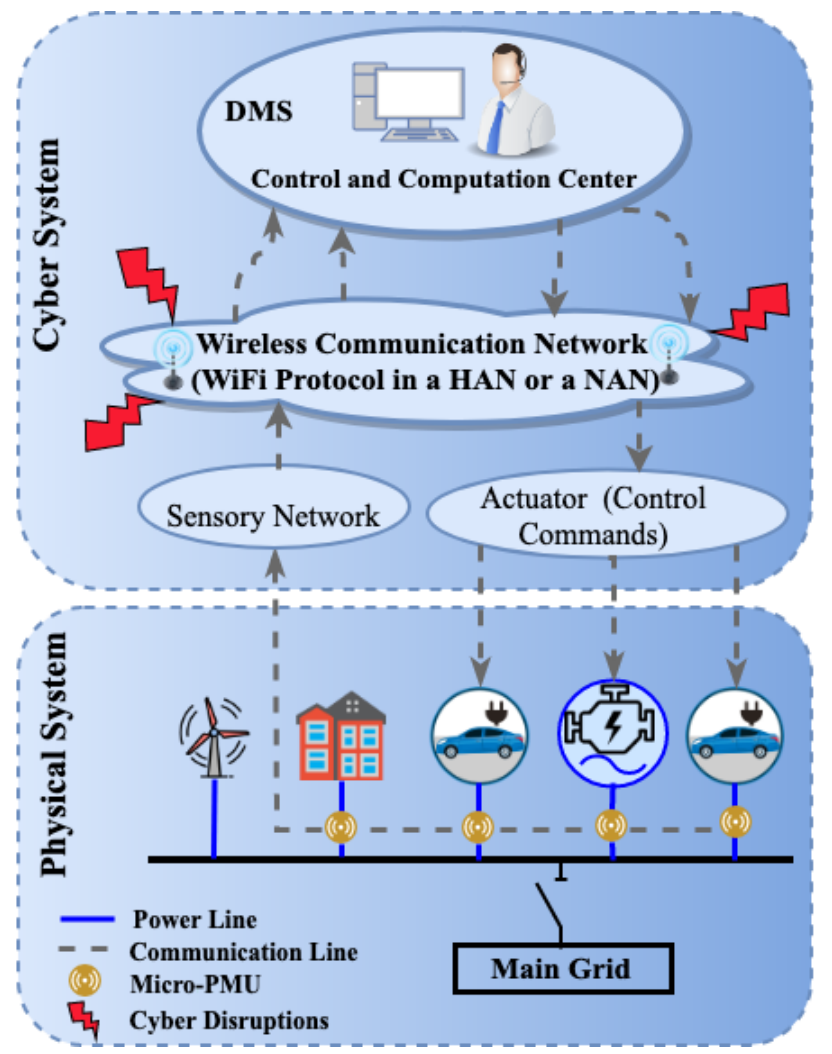

Figure 4.1: Cyber-Physical Power System Scheme of A Microgrid

the actuator of EVS.

This section presents a two-layer augmented Linear Quadratic Regulator (LQR) with UIO for extenuating the frequency deviations while improving system adaptiveness and resilience to uncertainties from wind energy and false data injection. In order to show the scalability of the proposed methodology, This controller is implemented on a simple LFC model for a microgrid and an inverter-based microgrid model which are introduced in this chapter. The contributions of the chapter are as follows:

1. It proposes a two-layer optimal augmented controller with the observer to mitigate the cyber disruptions using two-layer controller. In the first layer, the proposed observer not only estimates the microgrid states but also detects the UIs.

2. It shows that the proposed control strategy can mitigate the frequency deviation resulted from fluctuations arising from intermittent energy resources and load changes. It also determines that the proposed two-layer controller is robust against microgrid parameter changes.

3. It proves the stability of the proposed control strategy under uncertainties using the Lyapunov stability theory. Furthermore, it identifies the necessary conditions to detect and estimate unknown inputs in real-time while the systems are under measurement noises. 
4. It presents the suppression of frequency deviation using the proposed optimal controller in the second layer. The deviation is eliminated by a Linear Quadratic Regulator using the first layer detected UIs and estimated microgrid dynamics. This regulator also utilizes less energy than conventional controllers.

5. This work model an intelligent type of FDI affecting CPM. An effective manipulation considering the knowledge of control signals can deteriorate performance as seen by eigenvalue and vulnerability analysis. The magnitude and duration of FDI parameters are critically selected.

The rest of the section is organized as follows. Section 2 includes the state-space model of the CPM. Vulnerability and instability analysis is investigated in Section 3. The proposed observer and controller are described in Section 4. Simulation and results are demonstrated in Section 5, and the conclusion is presented in Section 6 .

\subsection{LFC Model for a Microgrid}

The section considers an isolated and small microgrid as shown in Figure 4.1, which includes a Diesel Generator (DG), an AC load, and two EVS. Distribution Management System (DMS) installed at the control center supervises the microgrid and communicates with $\mu \mathrm{PMUs}$ to receive state information. The DMS also sends logic processing and decisions from DMS to actuators of energy sources like diesel generator and EV stations, thus creating a cyber infrastructure for the CPM. The substantial use of communication for information interchange surrenders the CPM to risks by providing a medium of disruptions and intrusions. To examine the CPM vulnerability, the microgrid is modeled considering its controls as described in the following subsections [119].

\subsubsection{The State-Space Model of Microgrid}

The dynamic model of the LFC for a microgrid is represented in (1) and expanded in (2-4) where $x(t)$ is the state variable; $u(t)$ is the input control signal; $d(t)=\left[\begin{array}{ll}d_{1}(t) & d_{2}(t)\end{array}\right]$ is UI signal (disturbance) which models wind power perturbation $d_{1}(t)=\Delta P_{w}(t)$ and FDI that compromises EVS control input $d_{2}(t)$; and $y(t)$ is the control output signal. In the state vector (3), $\Delta f$ is the frequency deviation; $\Delta P_{D G}$ is the output power of DG, $\Delta X_{G}$ is the incremental valve position of governor, and $\Delta P_{E 1}$ and $\Delta P_{E 2}$ are the output powers of the first and second EVS, and $2 H_{t}$ denotes the equivalent inertia [119]. The relevant microgrid parameters can be found in [119]. The microgrid model considers an equivalent EVS dynamic model, where $T_{e}$ denotes time constant, and $\Delta u_{E}$ denotes the control inputs of the EVS with consideration of mechanical limits of the power ramp rate $\delta_{e}$, power increment $\mu_{e}$ and maximum $E_{\max }$ and minimum $E_{\text {min }}$ controllable energy [119]. [99] discusses the detailed information regarding EVS modeling.

$$
\left\{\begin{array}{l}
\dot{x}(t)=A x(t)+B u(t)+D d(t) \\
y(t)=C x(t)
\end{array}\right.
$$




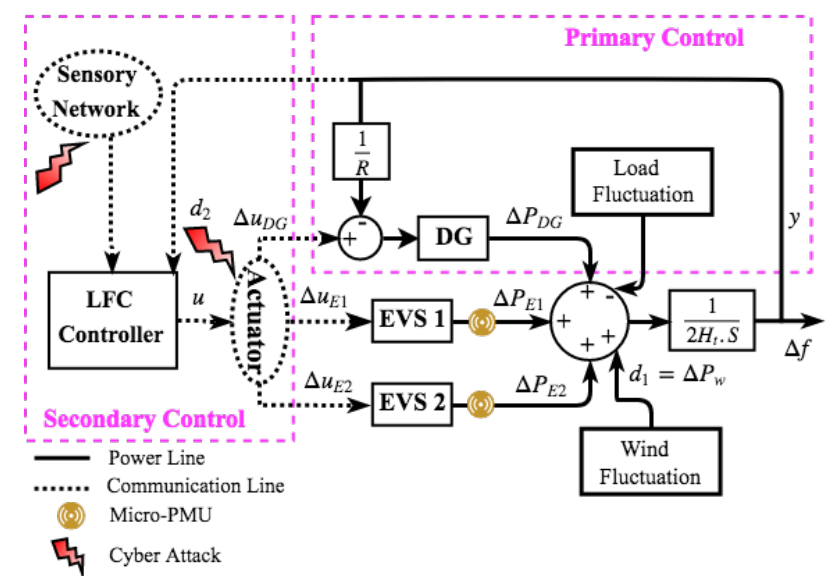

Figure 4.2: Diagram of Load Frequency Control

$$
\begin{aligned}
& A=\left[\begin{array}{ccccc}
0 & \frac{1}{2 H_{t}} & 0 & \frac{1}{2 H_{t}} & \frac{1}{2 H_{t}} \\
0 & \frac{-1}{2 T_{d}} & \frac{1}{T_{g}} & 0 & 0 \\
\frac{-1}{R T_{g}} & 0 & \frac{-1}{T_{g}} & 0 & 0 \\
0 & 0 & 0 & \frac{-1}{T_{e 1}} & 0 \\
0 & 0 & 0 & 0 & \frac{-1}{T_{e 2}}
\end{array}\right] \\
& B=\left[\begin{array}{ccc}
0 & 0 & 0 \\
0 & 0 & 0 \\
\frac{1}{T_{g}} & 0 & 0 \\
0 & \frac{1}{T_{e 1}} & 0 \\
0 & 0 & \frac{1}{T_{e 2}}
\end{array}\right] \\
& C=\left[\begin{array}{lllll}
1 & 0 & 0 & 0 & 0 \\
0 & 0 & 0 & 1 & 0 \\
0 & 0 & 0 & 0 & 1
\end{array}\right] \\
& D=\left[\begin{array}{ccccc}
-\frac{1}{2 H_{t}} & 0 & 0 & 0 & 0 \\
0 & 0 & 0 & -\frac{1}{T_{e 1}} & 0
\end{array}\right]^{T} \\
& x(t)=\left[\begin{array}{lllll}
\Delta f & \Delta P_{D G} & \Delta X_{G} & \Delta P_{E 1} & \Delta P_{E 2}
\end{array}\right]^{T} \\
& u=\left[\begin{array}{lll}
\Delta u_{D G} & \Delta u_{E 1} & \Delta u_{E 2}
\end{array}\right]^{T}, \\
& d=\left[\begin{array}{ll}
d_{1} & d_{2}
\end{array}\right]^{T}
\end{aligned}
$$

The DG adjusts the power output by the control input $\Delta u_{D G}$ with mechanical characteristics, such as speed regulation coefficients $R$, the DG time constant $T_{d}$ and the governor time constant $T_{g}$, and limits of the power ramp rate $\delta_{d g}$ and the power increment $\mu_{d g}[99]$. The microgrid control structure is explained in the next subsection. 


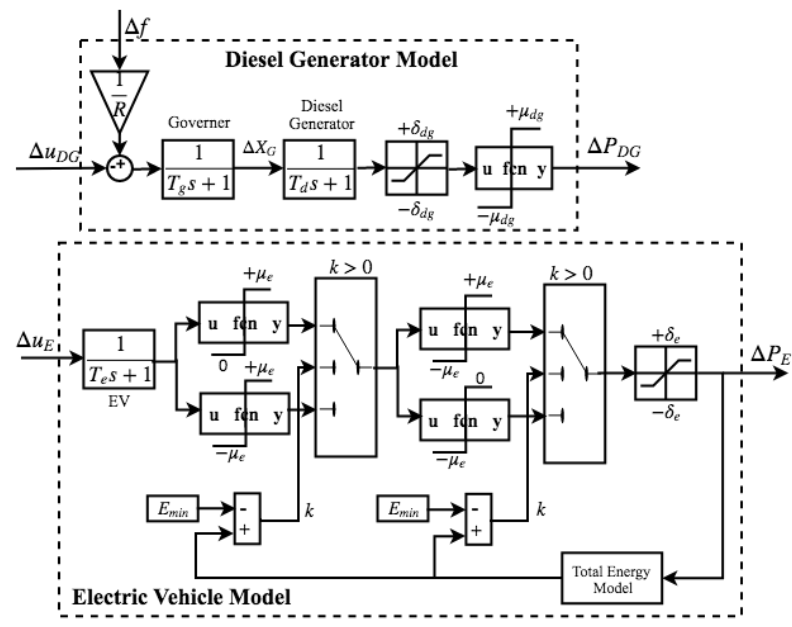

Figure 4.3: The diesel generator and electric vehicle model in LFC of the microgrid

\subsubsection{The Microgrid Control Structure}

The LFC in Figure 4.2 maintains the power supply and load balance. The input of LFC controller is frequency deviation $\Delta f(k)$, and its outputs $\Delta u_{D G}(k), \Delta u_{E 1}(k)$ and $\Delta u_{E 2}(k)$ serve as control inputs to DG and both EVS' respectively. The updated power outputs from EVS' and DG together with wind uncertainty $d_{1}(k)$ are subtracted from the demand to form the input of the swing equation whose output is the frequency deviation at each instant [119]. As illustrated in Figure 4.2, two control responses, primary and secondary, are considered to mitigate the frequency deviation. The primary control alters the mechanical settings of the governor of diesel generator $\Delta X_{G}(k)$ to adjust the generated power of DG. The controller has a limited dependency on cyber and communication infrastructure [100]; hence, the corresponding cyber threats are not discussed in this work. The secondary control fine-tunes the frequency utilizing EVS'. As shown in Figure 4.2, secondary control receives the system measurements from the sensory network and accordingly sends the control commands $\Delta u_{E 1}(k)$ and $\Delta u_{E 2}(k)$ to actuate EVS' to inject or absorb restorative power. The detailed model for both DG and EVS is shown in Figure 4.3. If this control commands $\Delta u_{E}(k)$ that are delivered through cyber platforms are corrupted by FDI $d_{2}(k)$, the microgrid is subject of actuator disruptions whose hazardous impacts are investigated in the next section.

\subsection{Vulnerability and Instability Analysis}

To show that actuator disruptions in EVS control can destabilize the CPM, the following proof of instability is used. Linear Time-Invariant (LTI) system is used and its optimal controller has the form of $u(t)=-G_{1} x(t)$ and the disrupted form is as shown in (4.5).

$$
u(t)= \begin{cases}-G_{1} x(t)+\delta G_{1} x(t) & t_{a}<t \leq t_{b} \\ -G_{1} x(t) & \text { Otherwise }\end{cases}
$$




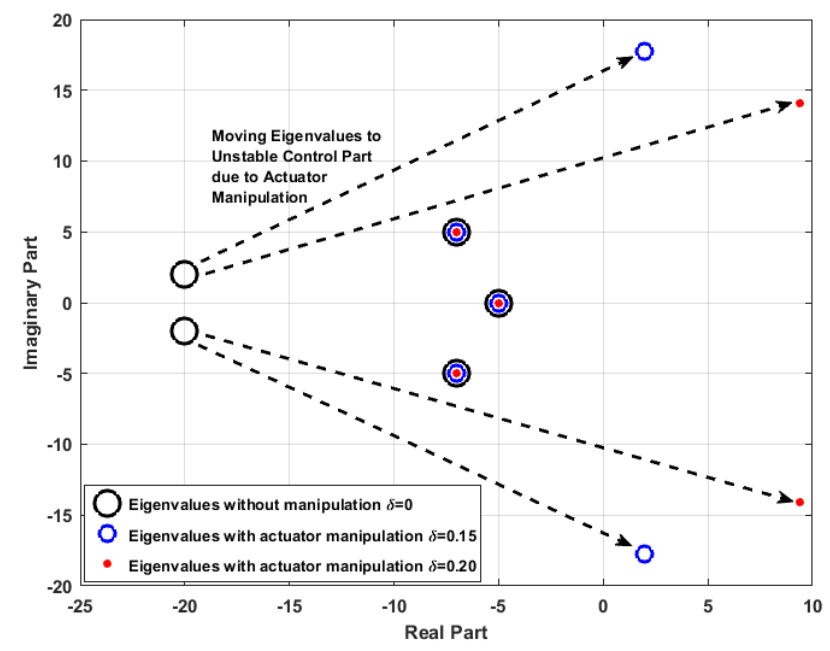

Figure 4.4: Eigenvalues of the system for normal operation and under different FDI disruptions

where $\delta$ is the magnitude of FDI that is occurring at time $t_{a}$ indicating stability issues at $t>t_{a}$. For simplicity, we consider $t_{b}=\infty$. In theorem 1 , we prove that this stealthy type of FDI creates a large impact on the microgrid and degrades the stability by affecting the eigenvalues of the system.

Theorem 1: Cyber disruptions are modeled as $d(t)$ and adversely impact the first and fourth microgrid states, respectively. To violate the safety bounds, the disruption is crafted based on dynamic knowledge of the system; therefore, $d(t)$ is defined as a function of $x(t)$ for the most undesirable consequence. The system operates under these manipulations in $d(t)$ is not stable if the corresponding dynamic model has at least one positive eigenvalue or at least one pole in the right-half plane.

Proof: Consider $D d(t)$ in (4.6) which models cyber disruption effect in the controller and can be written as:

$$
\dot{x}(t)=A x(t)+B u(t)+D\left(\delta G_{d} u(t)\right)
$$

Since $u(t)=-G_{1} x(t), d(t)$ can be written as $d(t)=\delta G_{d}\left(-G_{1} x(t)\right)$, then (4.6) can be simplified as $\dot{x}(t)=A x(t)-B G_{1} x(t)+D\left(\delta G_{d}\left(-G_{1} x(t)\right)\right)$ which is expressed in (4.7):

$$
\dot{x}(t)=\left(A-B G_{1}-D \delta G_{d} G_{1}\right) x(t)
$$

Therefore, the state dynamics in (7) for $t>t_{a}$ can be described by (8):

$$
x(t)=e^{\left(A-B G_{1}-D \delta G_{1}\right) t} X_{0}
$$

where $X_{0}$ is a vector of the initial values for the system states. It can be noted that, if $\Re(A-B K-D \delta K) \geq 0$, the system is unstable, indicating at least one positive eigenvalue in the system. This happens when $\delta=0.15$ to $\delta=0.2$ as shown in Figure 4.4 and increasing magnitudes make the microgrid more unstable. 


\subsection{Inverter-Based Microgrid Model}

In this section, the inverter-based microgrid model equipped with primary control is briefly introduced, the optimal secondary control implemented on this model is investigated. Finally the resiliency of the proposed UIO based controller leveraged for this model is examined in Section V.

\subsubsection{The Microgrid State-Space Representation}

This microgrid model consists of three main modules of inverters, network (line topology), and loads. Inverters dynamics comprises power-sharing controller, output filter, coupling inductor, and current and voltage controller. Each inverter has its own reference frame, e.g., axis $(d-q)_{i}$ for $i$ th inverter, whose rotating frequency $\omega_{i}$ is adjusted by its power-sharing controller. The loads and the networks dynamic equations are represented based on a common reference frame with axis $(d-q)_{i}$ and rotating frequency $\omega_{\text {com }}$, which is the reference frame of one of the microgrid inverters. The other inverters' reference frame can be translated to the common reference frame through the following transformation matrix [128].

$$
\begin{gathered}
{\left[f_{D Q}\right]=\left[T_{i}\right]\left[f_{d q}\right]} \\
{\left[T_{i}\right]=\left[\begin{array}{cc}
\cos \left(\delta_{i}\right) & -\sin \left(\delta_{i}\right) \\
\sin \left(\delta_{i}\right) & \cos \left(\delta_{i}\right)
\end{array}\right]}
\end{gathered}
$$

where $\delta_{i}$ is the angle of the reference frame of $i$ th inverter with respect to the common reference frame. It is worth to note that all inverters discussed here are Voltage Source Inverters (VSI) which are often utilized to link distributed generators to the grids.

\section{VSI State-Space Model}

As Figure 4.5 shows, the DG inverter control process is divided into three different part, including power, voltage, and current control loops. The power control loop adjusts the magnitude and frequency for the fundamental component of the inverter's voltage based on the droop features set for the real and reactive powers. Voltage and current controllers are applied to attenuate high-frequency disturbances and provide adequate damping for the output $L C$ filter $[128,129]$. Since we mostly focus on frequency control of this islanded microgrid, we merely explain the VSI power controller here (not voltage and current controllers). For more information regarding the detailed model, $[128,130]$ can be referred.

Using the droop control for DG inverters, we imitate the governor operation of synchronous generators in power grids. Once there is a load increment, the frequency of the system is decreased. Also, once there is a voltage drop, the reactive power is adjusted accordingly. The power control diagram of VSIs is shown in Figure 4.6. Examining Figure 4.6, instantaneous active $\tilde{p}$ and reactive power $\tilde{q}$ are obtained 


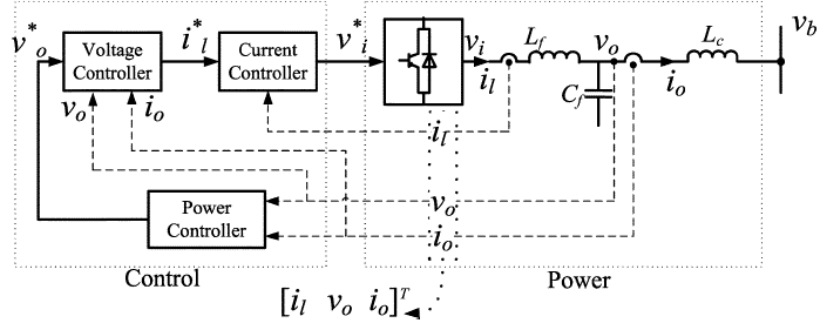

Figure 4.5: General diagram of DG inverter connected to Microgrids [128]

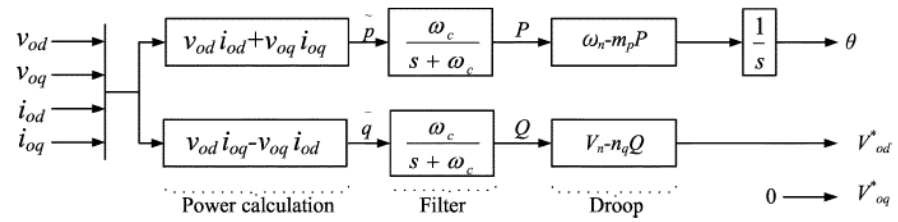

Figure 4.6: External power controller diagram of DG inverter [128]

from the measured voltage and current in $(d-q)$ frame as in $(4.11,4.12)$.

$$
\begin{aligned}
& \tilde{p}=v_{o d} i_{o d}+v_{o q} i_{o q} \\
& \tilde{q}=v_{o d} i_{o q}+v_{o q} i_{o d}
\end{aligned}
$$

Also, these instantaneous power components are passed through low-pass filters with $\omega_{c}$ cut-off frequency to achieve the fundamental active and reactive power as in (4.13, $4.14)$.

$$
\begin{aligned}
& P=\frac{\omega_{c}}{s+\omega_{c}} \tilde{p} \\
& Q=\frac{\omega_{c}}{s+\omega_{c}} \tilde{q}
\end{aligned}
$$

The artificial droop in the inverter frequency can share active and reactive powers. As seen in (4.15), the frequency $\omega$ is set based on the droop gain $m_{p}$, and the phase $\theta$ is set by integrating the frequency. $\omega_{n}$ denotes the nominal frequency set-point, and $\alpha$ denotes the inverter reference frame angle with nominal rotating frequency $\omega_{n}[128]$.

$$
\begin{gathered}
\omega=\omega_{n}-m_{p} P \\
\dot{\theta}=\omega, \quad \theta=\omega_{n} t-\int m_{p} P d t \\
\alpha=-\int m_{p} P d t, \quad \dot{\alpha}=-m_{p} P
\end{gathered}
$$

Similarly, an artificial droop is introduced to share the reactive power through choosing the output voltage magnitude which is aligned to the $d$-axis of the inverter reference frame (the $q$-axis reference is set to zero) as in (4.16). 


$$
v_{o d}^{*}=V_{n}-n_{q} Q, \quad v_{o q}^{*}=0
$$

The droop gains $m_{p}$ and $n_{q}$ are obtained using the maximum and minimum bounds of frequency and voltage magnitude as

$$
m_{p}=\frac{\omega_{\max }-\omega_{\min }}{P_{\max }}, \quad n_{q}=\frac{V_{\text {odmax }}-V_{\text {odmin }}}{Q_{\max }}
$$

As mentioned earlier, one of inverters' reference frame is considered as the common frame to obtain the complete model on a common reference frame. An angle $\delta$ is defined for every inverter as in (4.18) to transfer the variables of the different inverter into the common reference frame.

$$
\delta_{i}=\int\left(\omega_{i}-\omega_{c o m}\right) \quad \Delta \dot{\delta}_{i}=\Delta \omega_{i}-\Delta \omega_{c o m}=\Delta \omega_{n i}-\Delta \omega_{n 1}-\left(m_{i} \Delta P_{i}-m_{1} \Delta P_{1}\right)
$$

where $\omega_{n i}$ is the nominal frequency set-point for each inverter [131,132]. As seen in (4.18), all inverter angle dynamics $\dot{\Delta} \delta_{i}$ are a function of the first inverter active power $\Delta P_{1}$. After considering dynamics of voltage and current controllers and rearranging all equations, the combined small-signal model for " $s$ " number of DG inverters on a common reference frame is as in $(4.19,4.20)$.

$$
\begin{gathered}
{\left[\Delta \dot{x}_{I N V}\right]=A_{I N V}\left[\Delta x_{I N V}\right]+B_{I N V}\left[\Delta v_{b D Q}\right]+B_{c o m}\left[\Delta \omega_{c o m}\right]+B_{n}\left[\Delta \omega_{n}\right]} \\
{\left[\Delta i_{o D Q}\right]=C_{I N V n}\left[\Delta x_{I N V}\right]}
\end{gathered}
$$

where $\left[\Delta x_{I N V}\right]=\left[\begin{array}{llll}\Delta x_{i n v 1} & \Delta x_{i n v 2} & \ldots & \Delta x_{i n v s}\end{array}\right]$ and $\left[\Delta \omega_{n}\right]=\left[\begin{array}{lll}\Delta \omega_{n 1} & \Delta \omega_{n 2} & \ldots\end{array}\right.$ $\left.\Delta \omega_{n 3}\right]$.

\section{Network Model}

Assuming the microgrid with $n$ lines, $m$ nodes, $s$ inverters, and $p$ loads shown in Figure 4.7 is the case study for this section. The dynamic equations of line current of $i$ th line linked between nodes $j$ and $k$ on the common reference frame are obtained as $(4.21,4.22)[128]$.

$$
\begin{aligned}
& \frac{d i_{\text {lineDi }}}{d t}=\frac{-r_{\text {linei }}}{L_{\text {linei }}} i_{\text {lineDi } i}+\omega i_{\text {lineQ } i}+\frac{1}{L_{\text {linei }}} v_{b D j}-\frac{1}{L_{\text {linei }}} v_{b D k} \\
& \frac{d i_{\text {lineQ } i}}{d t}=\frac{-r_{\text {linei }}}{L_{\text {linei }}} i_{\text {lineQ } i}-\omega i_{\text {lineD } i}+\frac{1}{L_{\text {linei }}} v_{b Q j}-\frac{1}{L_{\text {linei }}} v_{b Q k}
\end{aligned}
$$

Therefore, the small-signal state-space model of the microgrid network can be generally obtained as in 4.23

$$
\left[\Delta i_{\text {line } D Q}^{\cdot}\right]=A_{N E T}\left[\Delta i_{\text {line } D Q}\right]+B_{1 N E T}\left[\Delta v_{b D Q}\right]+B_{2 N E T} \Delta \omega
$$




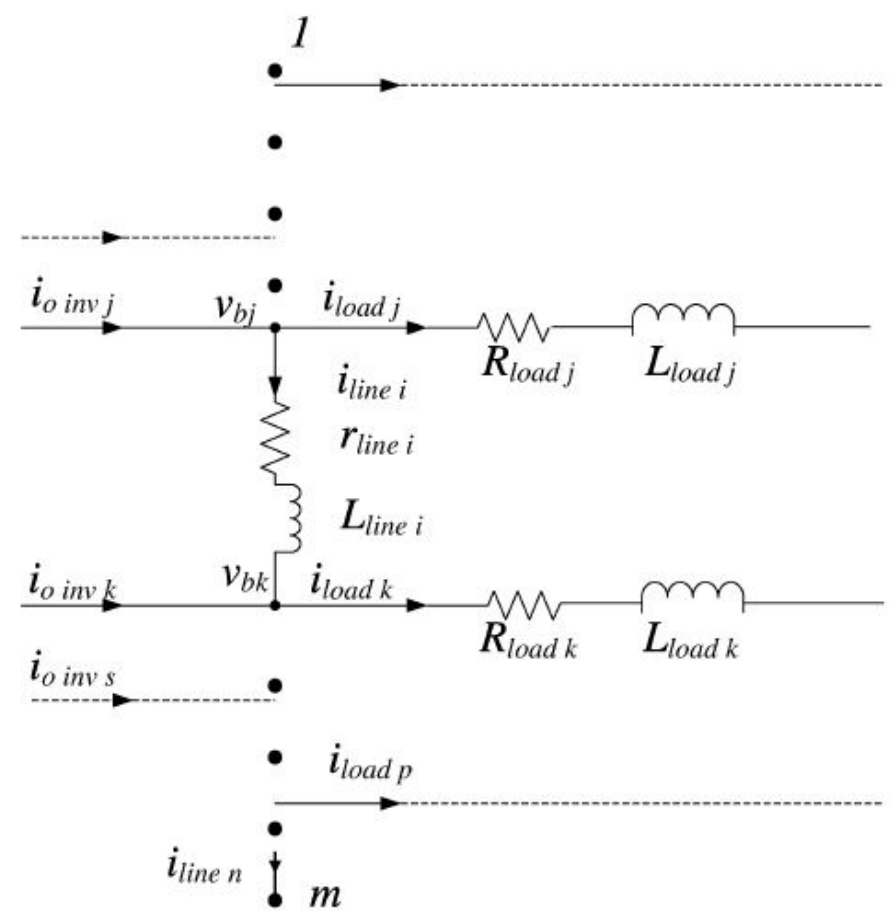

Figure 4.7: Network topology of the inverter-based microgrid model [128]

\section{Load Model}

The dynamic equation for the resistive and inductive load connected at the $i$ th node is obtained in (4.24)

$$
\begin{aligned}
& \frac{d i_{\text {loadDi }}}{d t}=\frac{-R_{\text {loadi }}}{L_{\text {loadi }}} i_{\text {loadD } i}+\omega i_{\text {loadQ } i}+\frac{1}{L_{\text {loadi }}} v_{b D i} \\
& \frac{d i_{\text {loadQ } i}}{d t}=\frac{-R_{\text {loadi }}}{L_{\text {loadi }}} i_{\text {loadQ } i}-\omega i_{\text {loadDi } i}+\frac{1}{L_{\text {loadi }}} v_{b Q i}
\end{aligned}
$$

Therefore, the small-signal state-space model of loads can be generally obtained as in $(4.26)$

$$
\left[\Delta i_{\text {loadDQ }}\right]=A_{\text {load }}\left[\Delta i_{\text {loadDQ }}\right]+B_{1 L O A D}\left[\Delta v_{b D Q}\right]+B_{2 L O A D} \Delta \omega
$$

\section{Complete Microgrid Model}

The microgrid model is obtained by merging all these modules of VSI inverters, network lines, and loads as in (4.27).

$$
\left[\begin{array}{c}
\Delta x_{I N V} \\
\Delta x_{N e t} \\
\Delta x_{\text {Load }}
\end{array}\right]=A_{M G}\left[\begin{array}{c}
\Delta x_{I N V} \\
\Delta x_{N e t} \\
\Delta x_{\text {Load }}
\end{array}\right]+B_{M G}\left[\Delta \omega_{n}\right]
$$


where $A_{M G}, B_{M G}$ are microgrid characteristic matrices and can be found in detail in [131]. Having this state-space representation of the microgrid, we can design an optimal secondary control for this system and compare the results of secondary and primary control of the microgrid.

\subsubsection{Secondary Control of the Inverter-based Microgrid}

In order to leverage an optimal control based on Linear Quadratic Regulator (LQR), we need to make sure the pair $\left(A_{M G}, B_{M G}\right)$ is controllable; otherwise, it is not possible to design such controllers for this system. However, this system is not controllable since the small-signal transient, and the steady-state responses obtained from the first state $\Delta \delta_{1}$ is zero $\left(\Delta \dot{\delta}_{1}=\Delta \omega_{1}-\Delta \omega_{\text {com }}=0\right)$ [133]. Therefore, this state must be discarded by removing the corresponding row and column in $A_{M G}$ and $B_{M G}$ or applying minimum realization technique for this system. After making the reduced-order model, we can apply the LQR controller on the microgrid model. LQR is explained in-detail in the next chapter. The microgrid eigenvalues before and after removing the state corresponding to $\Delta \delta_{1}$ are shown in Table 4.1.

\subsection{The Proposed Control Method}

Traditional observer-based controllers for microgrids and power systems utilize all the system states for the estimation and also control process. Frequency deviation $\Delta f$ is one of the states that is distorted due to uncertainties from renewable energy sources and loads resulting in poor closed-loop control. Joint SUIO and LQR controllers contain two layers that can obtain true frequency deviation even under uncertainties. The first layer estimates the microgrid states and identifies the UIs using (4.28-4.38), as shown in Figure 4.4 and is discussed in Section III.A. The second layer suppresses the frequency excursion using (4.39-4.41) and is discussed in Section III.B.

\subsubsection{Design of Stochastic Unknown Input Observer}

In SUIO, the states $x(k)=\left[\begin{array}{ll}x_{1} & x_{2}\end{array}\right]^{T}$ are decoupled into those corresponding to known inputs, $x_{1}(k)$ and unknown inputs, $x_{2}(k)$ [134]. Here, $x_{2}(k)$ state denotes the frequency deviation $\Delta f(k)$ which is affected by the UI. Using this decoupling states, we can separately estimate the states $x_{1}(k)$ which are not corresponding to the uncertainties (or UI) and then estimate the state $x_{2}(k)=\Delta f$ which is affected by the UI and detect the UI. In this case, the frequency deviation $\Delta f(k)$ does not distort the estimation process and in turn, the frequency control of the microgrid. For decoupling the states, a non-singular matrix $\Psi=\left[\begin{array}{ll}N & D\end{array}\right]$ is defined where $N$ is the arbitrary matrix chosen such that $\Psi$ is non-singular $\left(N \in \mathbb{R}^{n(n-q)}\right)$ [135]. This transfer matrix $\Psi$ is multiplied to both sides of (4.1) to obtain its equivalent (4.28). For this equivalent system, we obtain a new system state representation $\bar{x}=\left[\begin{array}{ll}\bar{x}_{1} & \bar{x}_{2}\end{array}\right]^{T}=\Psi^{-1} x=\Psi^{-1}\left[\begin{array}{ll}x_{1} & x_{2}\end{array}\right]^{T}$ with $\bar{x}_{1} \in \mathbb{R}^{n-q}$ and $\bar{x}_{2} \in \mathbb{R}^{q}$, and new 
Resilient Stochastic Control Strategies in Cyber-Physical Microgrids

Table 4.1: Eigenvalues of the microgrid ( $A_{M G}$ matrix)

\begin{tabular}{|c|c|c|}
\hline & $\begin{array}{l}\text { Standard Inverter-Based } \\
\text { Microgrid Model }\end{array}$ & $\begin{array}{l}\text { Reduced-Order Inverter- } \\
\text { Based Microgrid Model }\end{array}$ \\
\hline & $-9.44 \mathrm{e} 6+\mathrm{j} 3.14 \mathrm{e} 2$ & $-9.44 \mathrm{e} 6+\mathrm{j} 3.14 \mathrm{e} 2$ \\
\hline & $-9.44 \mathrm{e} 6-\mathrm{j} 3.14 \mathrm{e} 2$ & $-9.44 \mathrm{e} 6-\mathrm{j} 3.14 \mathrm{e} 2$ \\
\hline & $-3.63 \mathrm{e} 6+\mathrm{j} 3.14 \mathrm{e} 2$ & $-3.63 \mathrm{e} 6+\mathrm{j} 3.14 \mathrm{e} 2$ \\
\hline & $-3.63 \mathrm{e} 6-\mathrm{j} 3.14 \mathrm{e} 2$ & $-3.63 \mathrm{e} 6-\mathrm{j} 3.14 \mathrm{e} 2$ \\
\hline & $-2.85 \mathrm{e} 6+\mathrm{j} 3.14 \mathrm{e} 2$ & $-2.85 \mathrm{e} 6+\mathrm{j} 3.14 \mathrm{e} 2$ \\
\hline & $-2.85 \mathrm{e} 6-\mathrm{j} 3.14 \mathrm{e} 2$ & $-2.85 \mathrm{e} 6-\mathrm{j} 3.14 \mathrm{e} 2$ \\
\hline & $-2.94 \mathrm{e} 3+\mathrm{j} 7.38 \mathrm{e} 3$ & $-2.94 \mathrm{e} 3+\mathrm{j} 7.38 \mathrm{e} 3$ \\
\hline & $-2.94 \mathrm{e} 3-\mathrm{j} 7.38 \mathrm{e} 3$ & $-2.94 \mathrm{e} 3-\mathrm{j} 7.38 \mathrm{e} 3$ \\
\hline & $-2.79 \mathrm{e} 3+\mathrm{j} 6.84 \mathrm{e} 3$ & $-2.79 \mathrm{e} 3+\mathrm{j} 6.84 \mathrm{e} 3$ \\
\hline & $-2.79 \mathrm{e} 3-\mathrm{j} 6.84 \mathrm{e} 3$ & $-2.79 \mathrm{e} 3-\mathrm{j} 6.84 \mathrm{e} 3$ \\
\hline & $-2.84 \mathrm{e} 3+\mathrm{j} 4.89 \mathrm{e} 3$ & $-2.84 \mathrm{e} 3+\mathrm{j} 4.89 \mathrm{e} 3$ \\
\hline & $-2.84 \mathrm{e} 3-\mathrm{j} 4.89 \mathrm{e} 3$ & $-2.84 \mathrm{e} 3-\mathrm{j} 4.89 \mathrm{e} 3$ \\
\hline & $-2.53 \mathrm{e} 3+\mathrm{j} 4.43 \mathrm{e} 3$ & $-2.53 \mathrm{e} 3+\mathrm{j} 4.43 \mathrm{e} 3$ \\
\hline & $-2.53 \mathrm{e} 3-\mathrm{j} 4.43 \mathrm{e} 3$ & $-2.53 \mathrm{e} 3-\mathrm{j} 4.43 \mathrm{e} 3$ \\
\hline & $-2.86 \mathrm{e} 3+\mathrm{j} 2.92 \mathrm{e} 3$ & $-2.86 \mathrm{e} 3+\mathrm{j} 2.92 \mathrm{e} 3$ \\
\hline & $-2.86 \mathrm{e} 3-\mathrm{j} 2.92 \mathrm{e} 3$ & $-2.86 \mathrm{e} 3-\mathrm{j} 2.92 \mathrm{e} 3$ \\
\hline & $-2.21 \mathrm{e} 3+\mathrm{j} 2.20 \mathrm{e} 3$ & $-2.21 \mathrm{e} 3+\mathrm{j} 2.20 \mathrm{e} 3$ \\
\hline & $-2.21 \mathrm{e} 3-\mathrm{j} 2.20 \mathrm{e} 3$ & $-2.21 \mathrm{e} 3-\mathrm{j} 2.20 \mathrm{e} 3$ \\
\hline & $-1.49 \mathrm{e} 3+\mathrm{j} 2.51 \mathrm{e} 3$ & $-1.49 \mathrm{e} 3+\mathrm{j} 2.51 \mathrm{e} 3$ \\
\hline & $-1.49 \mathrm{e} 3-\mathrm{j} 2.51 \mathrm{e} 3$ & $-1.49 \mathrm{e} 3-\mathrm{j} 2.51 \mathrm{e} 3$ \\
\hline & $-1.29 \mathrm{e} 3+\mathrm{j} 2.10 \mathrm{e} 3$ & $-1.29 \mathrm{e} 3+\mathrm{j} 2.10 \mathrm{e} 3$ \\
\hline & $-1.29 \mathrm{e} 3-\mathrm{j} 2.10 \mathrm{e} 3$ & $-1.29 \mathrm{e} 3-\mathrm{j} 2.10 \mathrm{e} 3$ \\
\hline & $-1.31 \mathrm{e} 3+\mathrm{j} 1.71 \mathrm{e} 3$ & $-1.31 \mathrm{e} 3+\mathrm{j} 1.71 \mathrm{e} 3$ \\
\hline Eigenvalues & $-1.31 \mathrm{e} 3-\mathrm{j} 1.71 \mathrm{e} 3$ & $-1.31 \mathrm{e} 3-\mathrm{j} 1.71 \mathrm{e} 3$ \\
\hline & $-1.22 \mathrm{e} 3+\mathrm{j} 1.65 \mathrm{e} 3$ & $-1.22 \mathrm{e} 3+\mathrm{j} 1.65 \mathrm{e} 3$ \\
\hline & $-1.22 \mathrm{e} 3-\mathrm{j} 1.65 \mathrm{e} 3$ & $-1.22 \mathrm{e} 3-\mathrm{j} 1.65 \mathrm{e} 3$ \\
\hline & $-1.14 \mathrm{e} 3+\mathrm{j} 1.54 \mathrm{e} 3$ & $-1.14 \mathrm{e} 3+\mathrm{j} 1.54 \mathrm{e} 3$ \\
\hline & $-1.14 \mathrm{e} 3-\mathrm{j} 1.54 \mathrm{e} 3$ & $-1.14 \mathrm{e} 3-\mathrm{j} 1.54 \mathrm{e} 3$ \\
\hline & $-1.11 \mathrm{e} 3+\mathrm{j} 1.50 \mathrm{e} 3$ & $-1.11 \mathrm{e} 3+\mathrm{j} 1.50 \mathrm{e} 3$ \\
\hline & $-1.11 \mathrm{e} 3-\mathrm{j} 1.50 \mathrm{e} 3$ & $-1.11 \mathrm{e} 3-\mathrm{j} 1.50 \mathrm{e} 3$ \\
\hline & $-2.00 \mathrm{e} 1+\mathrm{j} 3.13 \mathrm{e} 2$ & $-2.00 \mathrm{e} 1+\mathrm{j} 3.13 \mathrm{e} 2$ \\
\hline & $-2.00 \mathrm{e} 1-\mathrm{j} 3.13 \mathrm{e} 2$ & $-2.00 \mathrm{e} 1-\mathrm{j} 3.13 \mathrm{e} 2$ \\
\hline & $-2.50 \mathrm{e} 1+\mathrm{j} 3.13 \mathrm{e} 2$ & $-2.50 \mathrm{e} 1+\mathrm{j} 3.13 \mathrm{e} 2$ \\
\hline & $-2.50 \mathrm{e} 1-\mathrm{j} 3.13 \mathrm{e} 2$ & $-2.50 \mathrm{e} 1-\mathrm{j} 3.13 \mathrm{e} 2$ \\
\hline & $-1.42 \mathrm{e} 2+\mathrm{j} 2.10 \mathrm{e} 2$ & $-1.42 \mathrm{e} 2+\mathrm{j} 2.10 \mathrm{e} 2$ \\
\hline & $-1.42 \mathrm{e} 2-\mathrm{j} 2.10 \mathrm{e} 2$ & $-1.42 \mathrm{e} 2-\mathrm{j} 2.10 \mathrm{e} 2$ \\
\hline & $-1.23 \mathrm{e} 2+\mathrm{j} 1.50 \mathrm{e} 2$ & $-1.23 \mathrm{e} 2+\mathrm{j} 1.50 \mathrm{e} 2$ \\
\hline & $-1.23 \mathrm{e} 2-\mathrm{j} 1.50 \mathrm{e} 2$ & $-1.23 \mathrm{e} 2-\mathrm{j} 1.50 \mathrm{e} 2$ \\
\hline & $-13.48+\mathrm{j} 30.21$ & $-13.48+\mathrm{j} 30.21$ \\
\hline & $-13.48-\mathrm{j} 30.21$ & $-13.48-\mathrm{j} 30.21$ \\
\hline & $-15.53+\mathrm{j} 10.59$ & $-15.53+\mathrm{j} 10.59$ \\
\hline & $-15.53-\mathrm{j} 10.59$ & $-15.53-\mathrm{j} 10.59$ \\
\hline & -20.84 & -20.84 \\
\hline & -28.25 & -28.25 \\
\hline & -31.38 & -31.38 \\
\hline & -31.40 & -31.40 \\
\hline & 0 & Removed \\
\hline
\end{tabular}


constant characteristic matrices defined in (4.29).

$$
\begin{gathered}
\left\{\begin{array}{l}
\bar{x}(k+1)=\bar{A} \bar{x}(k)+\bar{B} u(k)+\bar{D} d(k)+w(k) \\
y(k)=\bar{C} \bar{x}(k)+v(k)
\end{array}\right. \\
\bar{A}=\left[\begin{array}{ll}
\bar{A}_{11} & \bar{A}_{12} \\
\bar{A}_{21} & \bar{A}_{22}
\end{array}\right]=\Psi^{-1} A \Psi, \quad \bar{B}=\left[\begin{array}{ll}
\bar{B}_{1} & \bar{B}_{2}
\end{array}\right]^{T}=\Psi^{-1} B, \\
\bar{D}=\Psi^{-1} D, \quad \bar{C}=C \Psi=\left[\begin{array}{ll}
C N & C D
\end{array}\right]
\end{gathered}
$$

Further, the states $\bar{x}_{2}(k)$ corresponding to the UI are dropped to obtain unknown input-free microgrid as in $(4.30)[135,136]$.

$$
\left\{\begin{array}{l}
{\left[\begin{array}{ll}
I_{n-q} & 0
\end{array}\right] \bar{x}(k+1)=\left[\begin{array}{ll}
\bar{A}_{11} & \bar{A}_{12}
\end{array}\right] \bar{x}(k)+\bar{B}_{1} u(k)+w_{1}(k)} \\
y(k)=\left[\begin{array}{ll}
C N & C D
\end{array}\right] \bar{x}(k)+v(k)
\end{array}\right.
$$

where $w_{1}(k)$ is the noise which affects $\bar{x}_{1}(k)$. Assuming $\bar{x}_{2}(k)$ can be obtained from the measurement output $y(k),(4.30)$ will be transformed into a linear descriptor. In the transfer matrix $U=\left[\begin{array}{ll}C D & \Gamma\end{array}\right], C D$ is a full-column rank matrix and $\Gamma \in \mathbb{R}^{m \times(m-q)}$ is an arbitrary matrix defined such that $U$ is not a singular matrix. Hence we have $U^{-1}=\left[\begin{array}{ll}U_{1} & U_{2}\end{array}\right]^{T}$ with $U_{1} \in \mathbb{R}^{q \times m}$ and $U_{2} \in \mathbb{R}^{(m-q) \times m}$. Multiplying the measurement equation in the system (4.30) by $U^{-1}$, we obtain (4.31-4.32):

$$
\begin{gathered}
U_{1} y(k)=U_{1} C N \bar{x}_{1}(k)+\bar{x}_{2}(k) \\
U_{2} y(k)=U_{2} C N \bar{x}_{1}(k)
\end{gathered}
$$

Substituting (4.31) in (4.30) and combining it with (4.32), we get (4.33) with revised state matrix of $\widetilde{A}=\bar{A}_{11}-\bar{A}_{12} U_{1} C N$, revised measurement matrix $\widetilde{C}=U_{2} C N$, revised measurement vector $\bar{y}(k)=U_{2} y(k), v_{1}(k)$ is the noise which affects $\bar{y}(k)=$ $U_{2} y(k)$ and $E=\bar{A}_{12} U_{1}$.

$$
\left\{\begin{array}{l}
\bar{x}_{1}(k+1)=\widetilde{A} \bar{x}_{1}(k)+\bar{B}_{1} u(k)+E y(k)+w_{1}(k) \\
\bar{y}(k)=\widetilde{C} \bar{x}_{1}(k)+v_{1}(k)
\end{array}\right.
$$

If the pair $(\widetilde{A}, \widetilde{C})$ is observable, a Kalman filter can be designed for this system. The existence conditions for observability of system is examined in [135]. Considering the observability of the microgrid model and the noise covariance matrices $\operatorname{Cov}(w(k), w(k))=Q_{1}$ and $\operatorname{Cov}(v(k), v(k))=R_{1}$, Kalman filter with $L \in$ $\mathbb{R}^{(n-q) \times(m-q)}$ for $\bar{x}_{1}(k)$ is constructed as in (4.34) to estimate $\bar{x}_{1}(k)$ with $\hat{\bar{x}}_{1} \in \mathbb{R}^{n-q}$ :

$$
\hat{\bar{x}}_{1}(k+1 \mid k)=(\widetilde{A}-L \widetilde{C}) \hat{\bar{x}}_{1}(k \mid k-1)+\bar{B} u(k)+L^{*} \bar{y}(k)
$$

where $L^{*}=L U_{2}+E$ and the Kalman filter gain matrix $L$ is derived by solving the discrete Riccati equation $L=\left(A P_{1} C^{T}\right)\left(C P_{1} C^{T}+Q_{1}+R_{1}\right)$. Also, $P_{1}$ solves the algebraic Riccati equation which minimizes the steady-state error covariance 
$P_{1}=\lim _{k \rightarrow \infty} E\left[\left(x_{1}-\hat{\bar{x}}_{1}\right)\left(x_{1}-\hat{\bar{x}}_{1}\right)^{T}\right]$. Now, the entire estimated states can be obtained in (4.35) using (4.31) and (4.34):

$$
\hat{x}(k \mid k)=\Psi \hat{\bar{x}}=\Psi\left[\begin{array}{l}
\hat{x}_{1}(k \mid k) \\
\hat{\bar{x}}_{2}(k \mid k)
\end{array}\right]
$$

where $\hat{\bar{x}}_{2}(k \mid k)=U_{1} y(k)-U_{1} C N \hat{\bar{x}}_{1}(k \mid k)$. This implies that the frequency deviation $x_{2}(k)=\Delta f(k)$ can be estimated from measurements and remaining estimated states $x_{1}(k)$ and measurements $y(k)$.

To identify the unknown input $d(k)$, including wind power as well as load profiles, (4.35) is substituted in (4.28) to obtain (4.36):

$$
\begin{aligned}
& {\left[\begin{array}{c}
\hat{\bar{x}}_{1}(k+1 \mid k) \\
U_{1} y(k+1)-U_{1} C N \hat{\bar{x}}_{1}(k+1 \mid k)
\end{array}\right]=} \\
& {\left[\begin{array}{ll}
A_{11} & A_{12} \\
A_{21} & A_{22}
\end{array}\right]\left[\begin{array}{c}
\hat{\bar{x}}_{1}(k \mid k) \\
U_{1} y(k)-U_{1} C N \hat{\bar{x}}_{1}(k \mid k)
\end{array}\right]+\left[\begin{array}{c}
\bar{B}_{1} \\
\bar{B}_{2}
\end{array}\right] u(k)+\left[\begin{array}{c}
0 \\
I_{q}
\end{array}\right] \hat{d}(k)}
\end{aligned}
$$

Simplifying (4.36), the UI can be detected as $\hat{d}(k)=U_{1} y(k+1)+U_{1} C N \hat{\bar{x}}_{1}(k+$ $1 \mid k)-\bar{A}_{12} \hat{\bar{x}}_{1}(k \mid k)-\bar{A}_{22}\left(U_{1} y(k)-U_{1} C N \hat{\bar{x}}_{1}(k \mid k)\right)-\bar{B}_{2} u(k)$. The comprehensive UI detection, uncertainties, is presented in (4.37):

$$
\hat{d}(k)=F_{1} \bar{y}(k+1)+F_{2} \hat{\bar{x}}_{1}(k \mid k)+F_{3} y(k)+F_{4} u(k)
$$

where $F_{1}=U_{1}, F_{2}=U_{1} C N L U_{2} C N+U_{1} C N \bar{A}_{12} U_{1} C N-U_{1} C N \bar{A}_{11}-\bar{A}_{21}+\bar{A}_{22} U_{1} C N$, $F_{3}=-U_{1} C N L U_{2}-U_{1} C N \bar{A}_{12} U_{1}-\bar{A}_{22} U_{1}$, and $F_{4}=-U_{1} C N \bar{B}_{1}-\bar{B}_{2}, \bar{y}$ is a filtered signal of $y$. Since the first term in the right-hand side of $(4.37), F_{1} y(k+1)$, is a derivative type, it increases the noise magnitude of measurements and leads to high distortions in frequency response. Thus a moving average filter (MAF) with $M$ windows, which is equal to 20 here, is designed to make the measurement signals $y(k+1)$ more smooth as in (4.38).

$$
\bar{y}(k+1)=\frac{1}{M} \sum_{i=0}^{M} y(k+1-i)
$$

These estimated microgrid states $\hat{x}(k)$ and the detected UIs $\hat{d}(k)$ are adopted in the compensating procedure of the second control layer introduced in the next subsection.

\subsubsection{Design of Unknown Input Compensator}

The optimal unknown input compensator is governed by $\hat{u}(k)=G_{1} \hat{x}(k)$ resulting in the closed-loop microgrid matrix $A-B G_{1} . G_{1}$ is an optimal state feedback gain which is generally chosen such that the eigenvalues of $A-B G_{1}$ are within the unit circle of the Z-plane for a stable closed-loop microgrid. For this, the pair $(A, B)$ must be controllable that means the rank of the controllability matrix is same as the system rank, or $\operatorname{rank}\left[\begin{array}{lllll}B & A B & A^{2} B & A^{3} B & A^{4} B\end{array}\right]=\operatorname{rank} A$. In the second layer, therefore, the optimal feedback controller gain $G_{1}$ is designed using input $u(k)$ and 

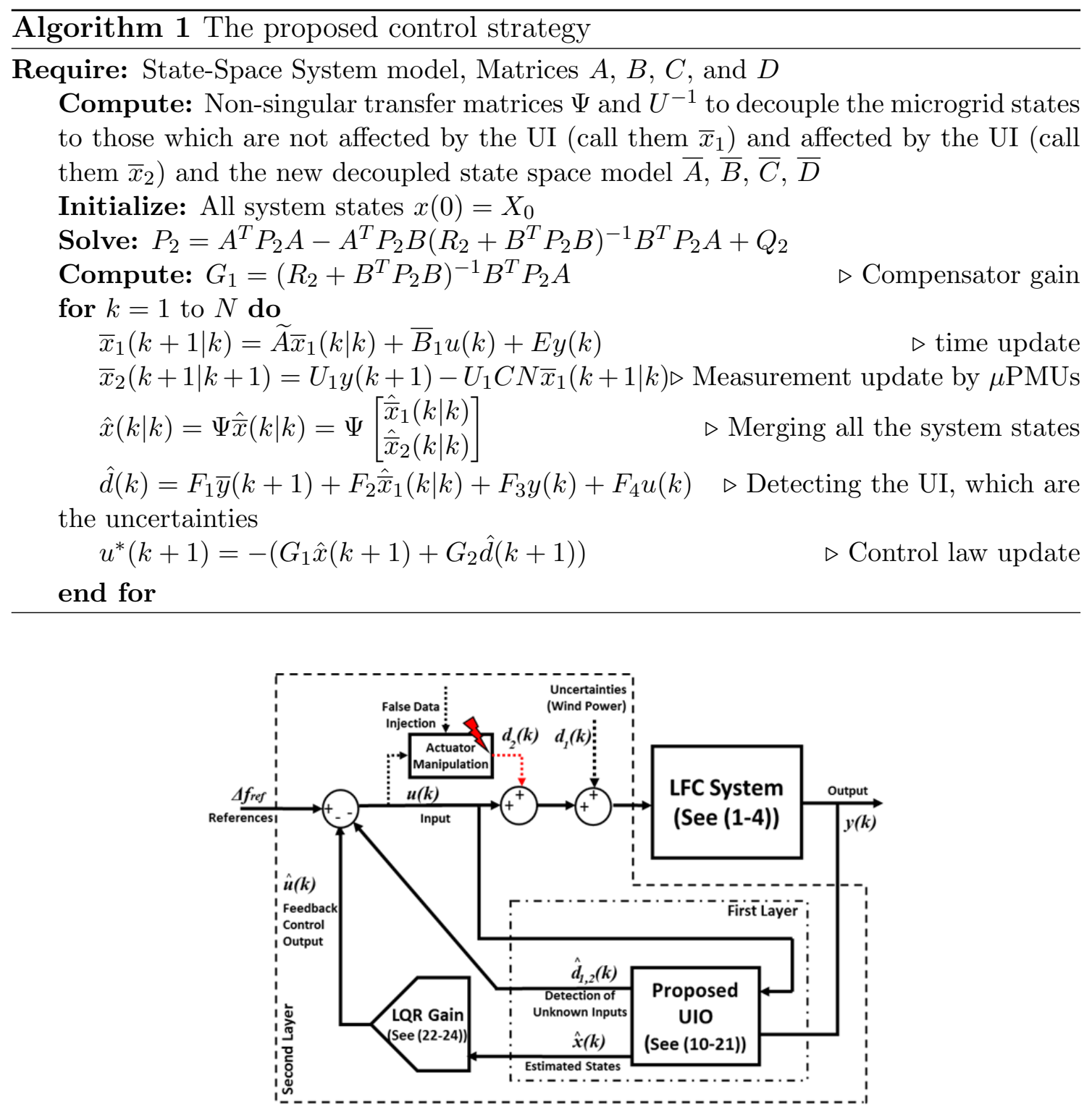

Figure 4.8: Load variation and wind power uncertainties (UI) [105]

the estimated microgrid states $\hat{x}(k)$ in (4.35) to minimize the performance index $J$ in (4.39). $Q_{2}$ and $R_{2}$ are weighting matrices corresponding to control performance and energy consumption, respectively, in (4.39). If $\Delta f_{r e f}=0$, the full control input $u^{*}(k)=-\hat{u}(k)-G_{2} \hat{d}(k)$ which is a linear combination of the detected UI $\hat{d}(k)$ with $G_{2}=D$, and the control gain $G_{1}$ in (4.40). In the first term of the full control input $u^{*}(k), G_{1}$, is calculated based on (4.41), and is designed to minimize $J$ using $P_{2}$, the unique positive-definite solution of discrete-time algebraic Riccati equation in (4.39) expressed as $P_{2}=A^{T} P_{2} A-A^{T} P_{2} B\left(R_{2}+B^{T} P_{2} B\right)^{-1} B^{T} P_{2} A+Q_{2}$, to obtain $G_{1}$ and the feedback control input $\hat{u}(k)$ for infinite-horizon. In the second term of the full control input $u^{*}(k)$, the UI is detected as $\hat{d}(k)$ by our detector expressed in the first layer of the proposed controller and subtracted from the feedback control law.. 


$$
\begin{gathered}
\text { Minimize } J=\sum_{n=1}^{\infty}\left(x(k)^{T} Q_{2} x(k)+u(k)^{T} R_{2} u(k)\right) \\
\text { Subject to. } \quad x(k+1)=A x(k)+B u(k) \\
u^{*}(k)=-\left(G_{1} \hat{x}(k)+G_{2} \hat{d}(k)\right) \\
G_{1}=\left(R_{2}+B^{T} P_{2} B\right)^{-1} B^{T} P_{2} A
\end{gathered}
$$

The proposed control strategy is elaborated in Algorithm 1.

\subsection{Stability Proof of the Proposed Methodology}

Stability of the proposed resilient control is investigated for the system described in (4.42).

$$
\left\{\begin{array}{l}
\dot{x}(t)=A_{c} x(t)+B_{c} u(t)+D d(t)+w(t) \\
y(t)=C x(t)+v(t)
\end{array}\right.
$$

Remark 1: The measurement noise $v(t)$ has an upper bound:

$$
\|v(t)\| \leq L_{O}
$$

Remark 2: The unknown input along with process noise $D d(t)+w(t)=S(t)$ is bounded by:

$$
\|S(t)\| \leq L_{S}
$$

Remark 3: The symmetric matrix $P_{L}$ has upper and lower limits:

$$
\lambda_{\min } I_{n} \leq P_{L} \leq \lambda_{\max } I_{n}
$$

where $\lambda_{\min }$ and $\lambda_{\max }$ are positive real numbers. $P_{L}$ can be found by solving the Lyapunov function:

$$
\left(A_{c}-L C\right)^{T} P_{L}+P_{L}\left(A_{c}-L C\right)=-Q_{L}
$$

where $Q_{L}$ is a symmetric positive-definite matrix. Consider the observer equation as:

$$
\begin{gathered}
\dot{\hat{x}}=A_{c} \hat{x}(t)+B_{c} u(t)+L(y-\hat{y})+D \hat{d}(t) \\
\hat{y}=C \hat{x}(t)
\end{gathered}
$$

where $L$ is the Kalman filter gain, and $\hat{d}(t)$ is the estimated unknown input. Subtracting Equation (4.44) from the Equation (4.42), estimate error of the system $\tilde{x}(t)$ can be found as:

$$
\begin{gathered}
\dot{\tilde{x}}(t)=\dot{x}(t)-\dot{\hat{x}}(t) \\
=\left(A_{c}-L C\right) \tilde{x}(t)+S(t)-\hat{S}(t)+w(t)+L(v(t))
\end{gathered}
$$

Corollary 1: we can prove that, $\|S(t)-\hat{S}(t)\| \leq L_{a}\|\tilde{x}(t)\|$, where $L_{a}$ is finite positive constant. The proof for convergence of the parameter updating process is described in [137]. 
Theorem 1: The system described in (4.42) remains stable and $\tilde{x}(t)$ in Equation (4.45) is bounded, if the following condition is satisfied $-\eta_{\min }\left(Q_{L}\right)+2 L_{a} \lambda_{\max }<0$, where $\eta$ and $\lambda$ are eigenvalues of $Q_{L}$ and $P_{L}$ respectively.

Proof: Consider the following Lyapunov function

$$
V(t)=\tilde{x}(t)^{T} P_{L} \tilde{x}(t)+\frac{1}{2} x(t)^{T} x(t)
$$

Since $V(t)$ is positive definite (based on Remark 3 ), $\dot{V}(t)$ needs to be negative definite, so that the system will be asymptotically stable. Derivative of $V(\tilde{x}, t)$ with respect to time can be written as:

$$
\dot{V}(t)=\dot{\tilde{x}}(t)^{T} P_{L} \tilde{x}(t)+\tilde{x}(t)^{T} P_{L} \dot{\tilde{x}}(t)+x(t)^{T} \dot{x}(t)
$$

Substituting $\dot{\tilde{x}}(t)$ in (4.45) into (4.48) results in

$$
\begin{gathered}
\dot{V}(t)=\left(A_{c} \tilde{x}(t)-L C \tilde{x}(t)+S(t)-\hat{S}(t)+w(t)+L(v(t))\right)^{T} P_{L} \tilde{x} \\
+\tilde{x}(t)^{T} P_{L}\left(A_{c} \tilde{x}(t)-L C \tilde{x}(t)+S(t)-\hat{S}(t)+w(t)+L(v(t))\right) \\
+x(t)^{T}\left(A_{c} x(t)+B u(t)+S(t)\right) \\
=\tilde{x}(t)^{T}\left(A_{c}-L C\right)^{T} P_{L} \tilde{x}(t)+\tilde{x}(t)^{T} P_{L}\left(A_{c}-L C\right) \tilde{x}(t) \\
+(S(t)-\hat{S}(t)+w(t)+L(v(t)))^{T} P_{L} \tilde{x} \\
+\tilde{x}^{T} P_{L}(S(t)-\hat{S}(t)+w(t)+L(v(t))) \\
+x(t)^{T}\left(A_{c} x(t)+B\left(-G_{1} x(t)-D \hat{d}+w(t)-w(t)\right)+S(t)\right) \\
=\tilde{x}(t)^{T}\left(A_{c}-L C\right)^{T} P_{L} \tilde{x}(t)+\tilde{x}(t)^{T} P_{L}\left(A_{c}-L C\right) \tilde{x}(t) \\
+(S(t)-\hat{S}(t)+w(t)+L(v(t)))^{T} P_{L} \tilde{x} \\
+\tilde{x}^{T} P_{L}(S(t)-\hat{S}(t)+w(t)+L(v(t))) \\
+x(t)^{T}\left(A_{c} x(t)+B\left(-G_{1} x(t)-\hat{S}(t)+w(t)+S(t)\right)\right. \\
\quad=-\tilde{X}(t) Q_{L} \tilde{X}(t) \\
+(S(t)-\hat{S}(t)+w(t)+L(v(t)))^{T} P_{L} \tilde{x} \\
+\tilde{x}^{T} P_{L}(S(t)-\hat{S}(t)+w(t)+L(v(t))) \\
+x(t)^{T}\left(\left(A_{c}-B G_{1}\right) x(t)-\hat{S}(t)+w(t)+S(t)\right) \\
\leq-Q_{L}\|\tilde{x}(t)\|^{2}+2\left\|P_{L}\right\| L_{a}\|\tilde{x}(t)\|^{2} \\
+\left(A_{c}-B G_{1}\right)\|x(t)\|^{2}+L_{a}\|x(t)\|^{2} \\
\leq\left(-\eta_{\min }\left(Q_{L}\right)+2 L_{a} \lambda_{\max }\right)\|\tilde{x}(t)\|^{2} \\
\quad+\left(L_{a}+\lambda_{1 \max }\right)\|x(t)\|^{2}
\end{gathered}
$$

The following conditions should be satisfied, in order to have a stable observer

$$
-\eta_{\min }\left(Q_{L}\right)+2 L_{a} \lambda_{\max }<0
$$

and to have a stable controller the following condition should be satisfied

$$
L_{a}+\lambda_{1 \max }<0
$$

where $\lambda_{1 \max }$ is the maximum eigenvalue of $A_{c}-B G_{1}$.

\subsection{Simulation and Results}

It is important for the proposed controller to resiliently perform under uncertainties arising from renewable energies, load, and microgrid parameter changes and 


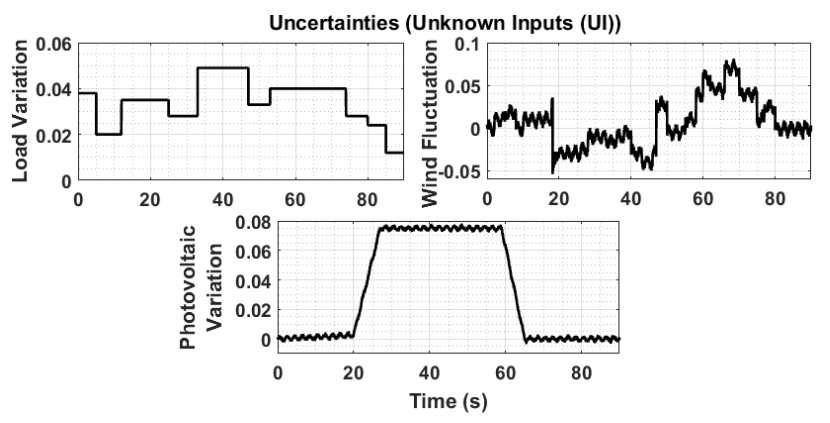

Figure 4.9: Load variation and wind power uncertainties (UI) [105, 138]

cyber disruptions. Also, it must be shown that this controller is scalable to other power systems. Therefore, the numerical results associated with this proposed control strategy are separately illustrated in three following subsections to show that this controller fulfills these mentioned concerns. The first and second subsections show that the proposed method robustly addresses uncertainties and also resiliently performs under FDI, respectively. In these two subsections, the proposed controller is implemented on LFC of a microgrid which has a small state-space representation with five states. In order to show the controller's performance in larger-scale in the third subsection, the proposed controller is utilized for an inverter-based microgrid model, which has a state-space representation with 47 states.

\subsubsection{The Proposed Controller Robustness Under Uncer- tainties}

This section presents the LFC results of the microgrid using the proposed twolayer controller designed in Section III. The performance of the proposed secondary SUIO and LQR controller is compared with that of, UIO and LQR described in our previous work [98], and Luenberger observer and LQR, as well as with results without the secondary control. These methodologies are implemented in MATLABSIMULINK. Three different cases are considered based on uncertainty considerations and the disconnection of EV stations. The data for the wind power fluctuation is extracted from an offshore wind farm in Sweden [105] and modeled using zero-mean profile over a period of 90 seconds as shown in Figure 4.9. The uncertainty of solar radiation in Aberdeen, UK [138] and load uncertainty considered are also shown in Figure 4.9. A measurement noise with zero-mean Gaussian and a covariance of 0.01 is considered for all measurements $y(k)$.

\section{Case 1}

In this case, the load characteristics in Figure 4.9 are considered in addition to the disconnection of EV stations at $t=33 \mathrm{~s}$. Frequency response without secondary control and with secondary control using three controllers is depicted in Figure 4.10. The control inputs (control efforts) $\Delta u_{D G}$ and $\Delta u_{E}$ for the DG and EV stations, is shown in Figure 4.11. It is clear that the proposed controller has a significantly 


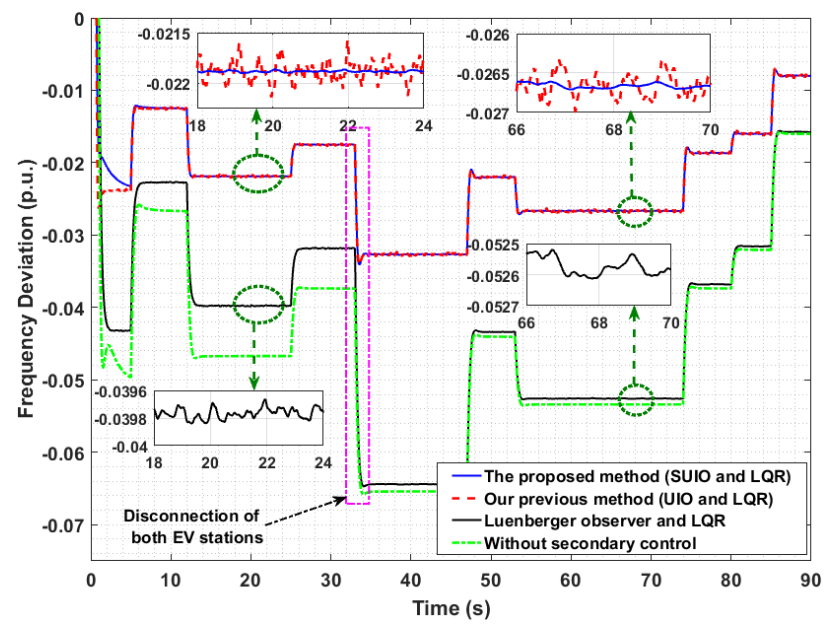

Figure 4.10: Frequency control response in case 1

better response than the other two controllers. The controller-based on Luenberger observer does not fully compensate the frequency deviations, especially when $t \geq 33 \mathrm{~s}$ during the disconnection of EV stations. At this time, the microgrid frequency drops by $0.224 p . u$. with the Luenberger observer-based controller and by $0.016 p . u$. with SUIO and the UIO-based controllers. The response of Luenberger-based controller is similar to the response without secondary control at $t \geq 33 \mathrm{~s}$. This shows that standard optimal controllers cannot perform well under severe contingencies such as loss of power and other uncertainties. It can be examined from Figure 4.11 that The Luenberger-based controller requires the most control efforts as compared to other controllers. Also, since UIO-based controller is not inherently designed to handle the measurement noise, its corresponding control efforts are more than that of the proposed SUIO controller. The total control effort $\Delta E_{t}=\Delta u_{D G}^{2}+\Delta u_{E 1}^{2}+\Delta u_{E 2}^{2}$ is as shown in the fourth subplot in Figure 4.11. As seen in Figure 4.11, $\Delta E_{t}=$ $1.5788 \mathrm{e}+03$ obtained from SUIO is significantly lower than $2.2506 \mathrm{e}+04$ obtained from UIO and LQR controller [98].

\section{Case 2}

In this case, the wind power fluctuations in Figure 4.9 are considered. Frequency responses without secondary control and with secondary control using three controllers are depicted in Figure 4.12. The control inputs (control efforts) for the DG $\Delta u_{D G}$ and EV stations $\Delta u_{E}$, are shown in Figure 4.13. It is clear that the proposed controller has a significantly better response than the other two controllers. The controller-based on Luenberger observer does not fully compensate the frequency deviations, and the maximum frequency deviation is 0.046 p.u.. The maximum frequency deviation is 0.0084 p.u. with SUIO and UIO-based controllers. It can be examined from Figure 4.13 that the Luenberger-based controller requires the most control efforts as compared to other controllers. The total control effort $\Delta E_{t}$ is as shown in the fourth subplot in Figure 4.13. As seen in Figure 4.11, $\Delta E_{t}=$ $1.6081 \mathrm{e}+03$ obtained from SUIO is significantly lower than $2.2569 \mathrm{e}+04$ obtained 


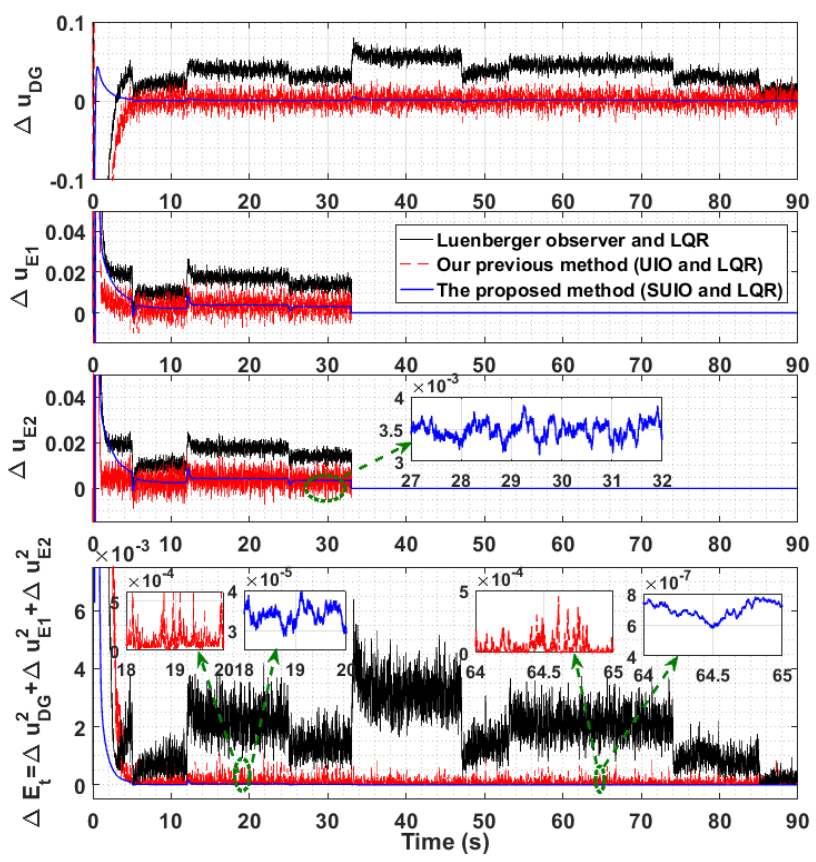

Figure 4.11: Control efforts in case 1

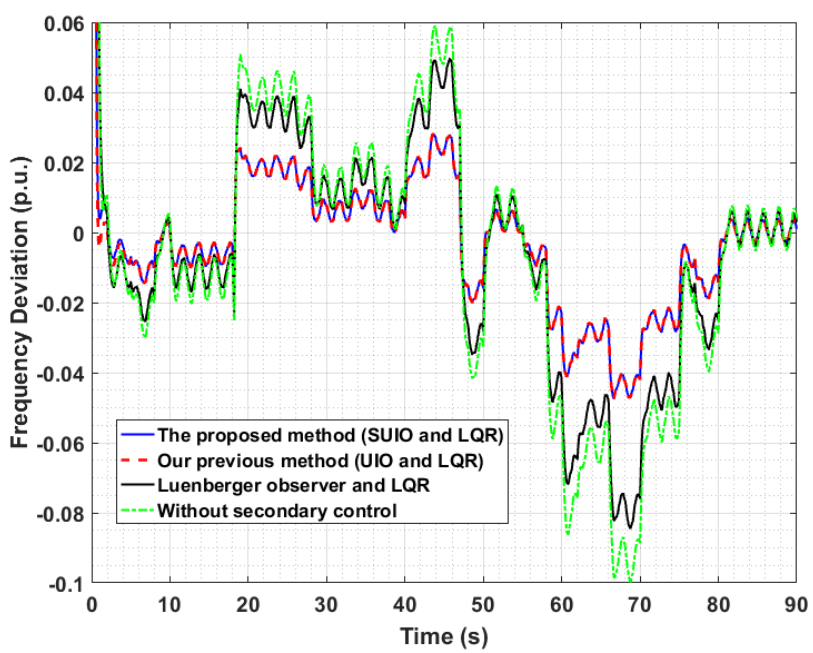

Figure 4.12: Frequency control response in case 2

from UIO and LQR controller [98].

\section{Case 3}

In this case, both wind power fluctuations and load characteristics in Figure 4.9 are considered in addition to the disconnection of one EV station at $t=16 \mathrm{~s}$. Frequency response without secondary control and with secondary control using three controllers is depicted in Figure 4.14. The control inputs (control efforts) $\Delta u_{D G}$ and $\Delta u_{E}$ for the DG and EV stations, are shown in Figure 4.15. It is clear that the proposed controller has a significantly better response than the other two controllers. 


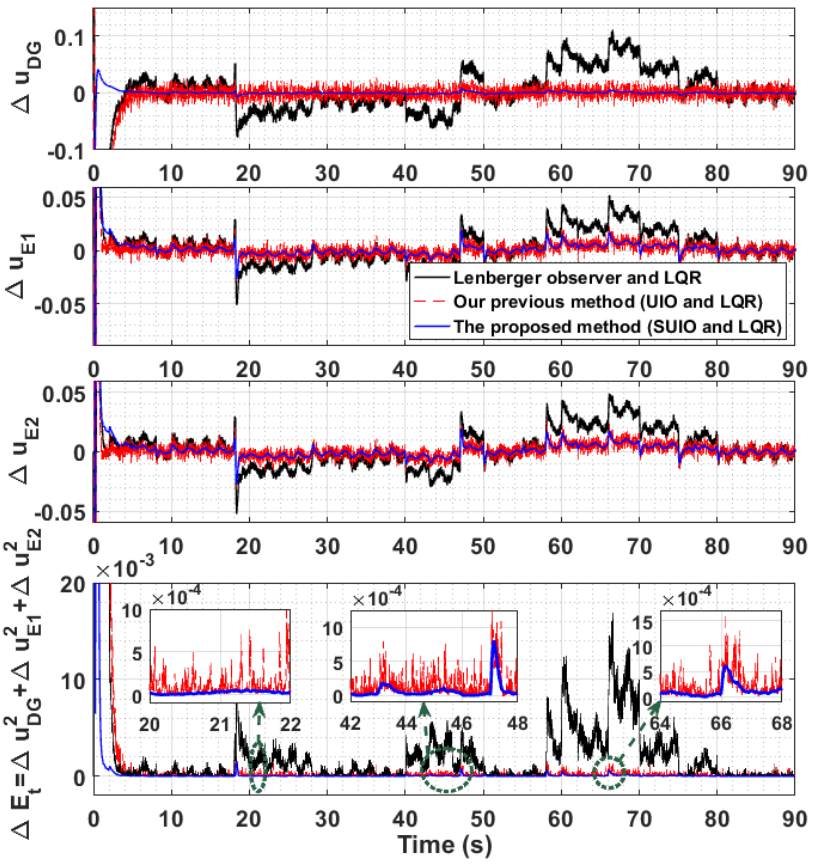

Figure 4.13: Control efforts in case 2

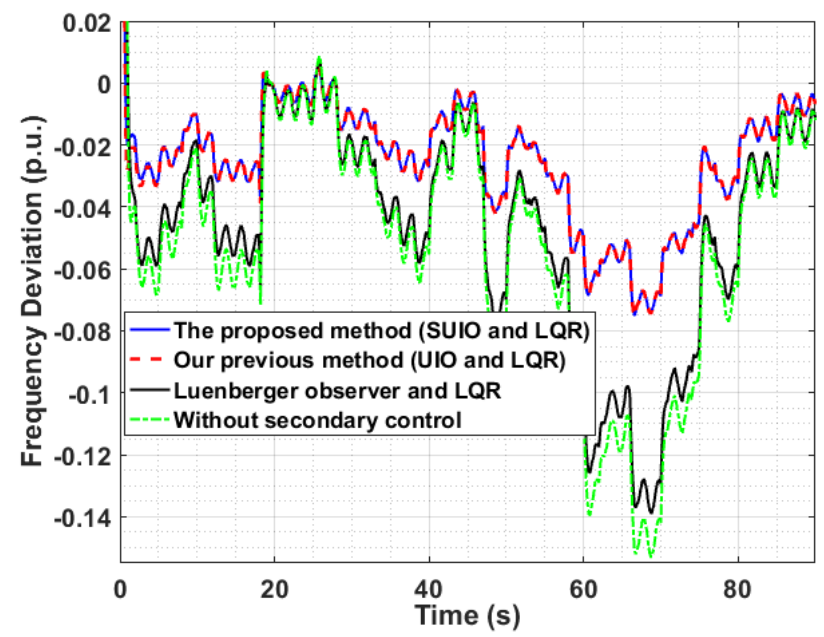

Figure 4.14: Frequency control response in case 3

The controller-based on Luenberger observer has a maximum frequency deviation of 0.14 p.u. which is higher than 0.075 p.u. obtained using SUIO and UIO based controllers. Figure 4.15 shows that the Luenberger-based controller requires the most control efforts as compared to other controllers. As seen in Figure 4.11, $\Delta E_{t}=$ $1.5991 \mathrm{e}+03$ obtained from SUIO is significantly lower than $2.2524 \mathrm{e}+04$ obtained from UIO and LQR controller [98]. As seen from the estimated states in Figure 4.16, the states from SUIO match very well the original states as compared to UIO. The performance of UIO is, in turn significantly better than a Luenberger-based observer controller. 

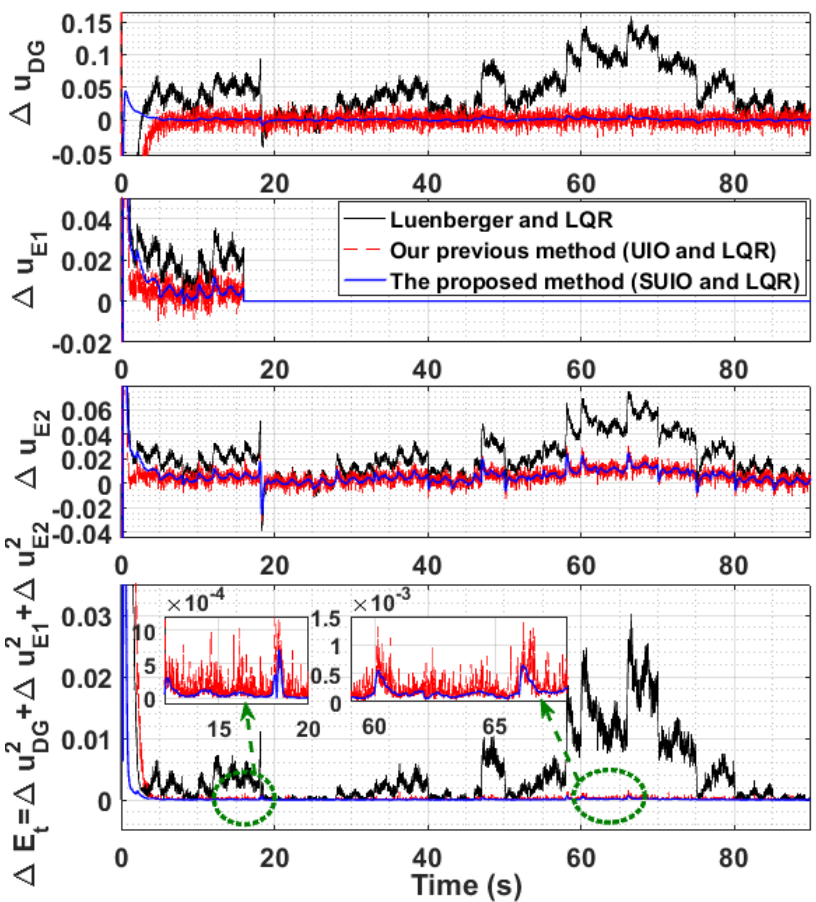

Figure 4.15: Control efforts in case 3
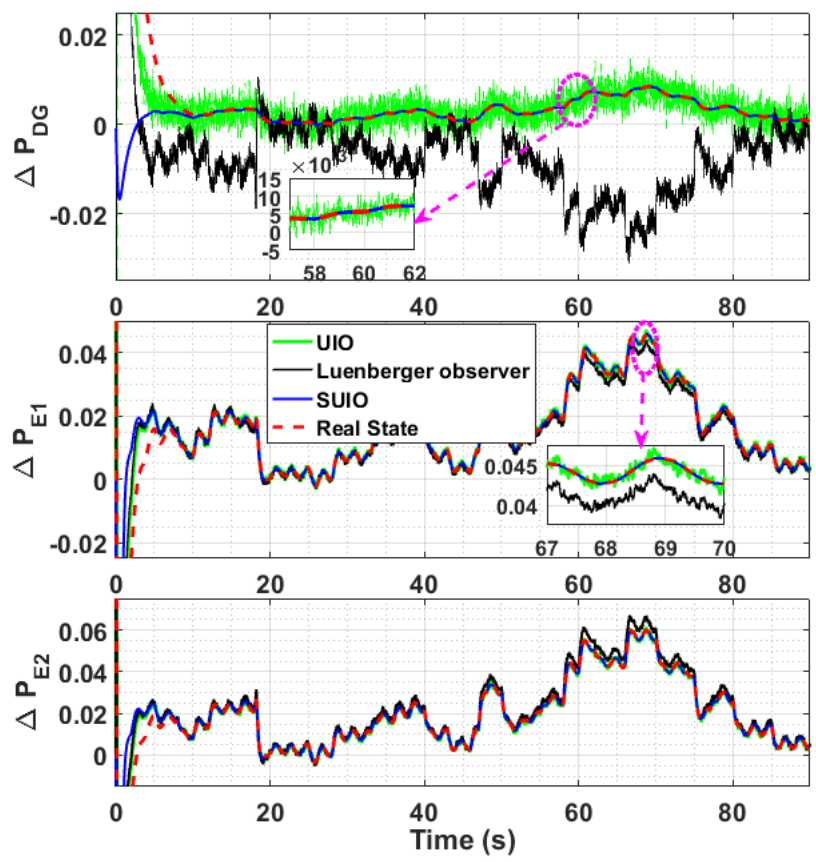

Figure 4.16: State estimation in case 3

\section{Case 4}

In this case, the photovoltaic intermittencies in Figure 4.17 are considered. Frequency responses without secondary control and with secondary control using three 


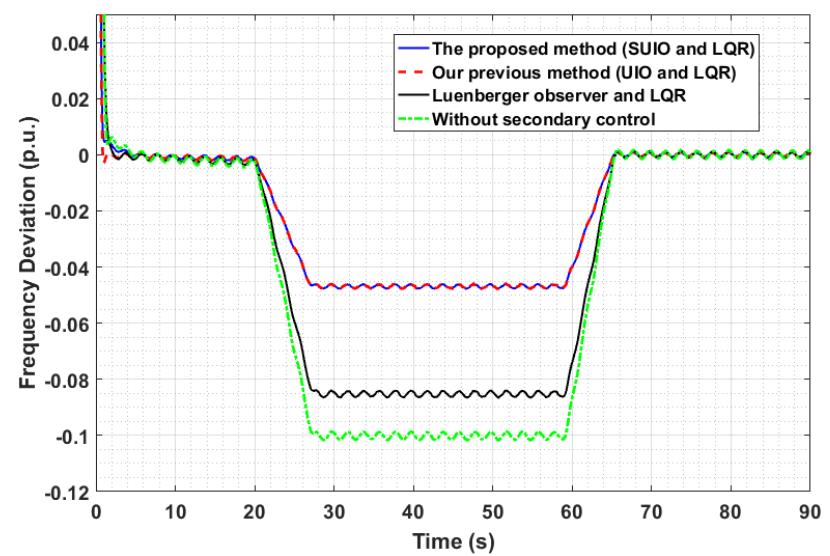

Figure 4.17: Control efforts in case 4

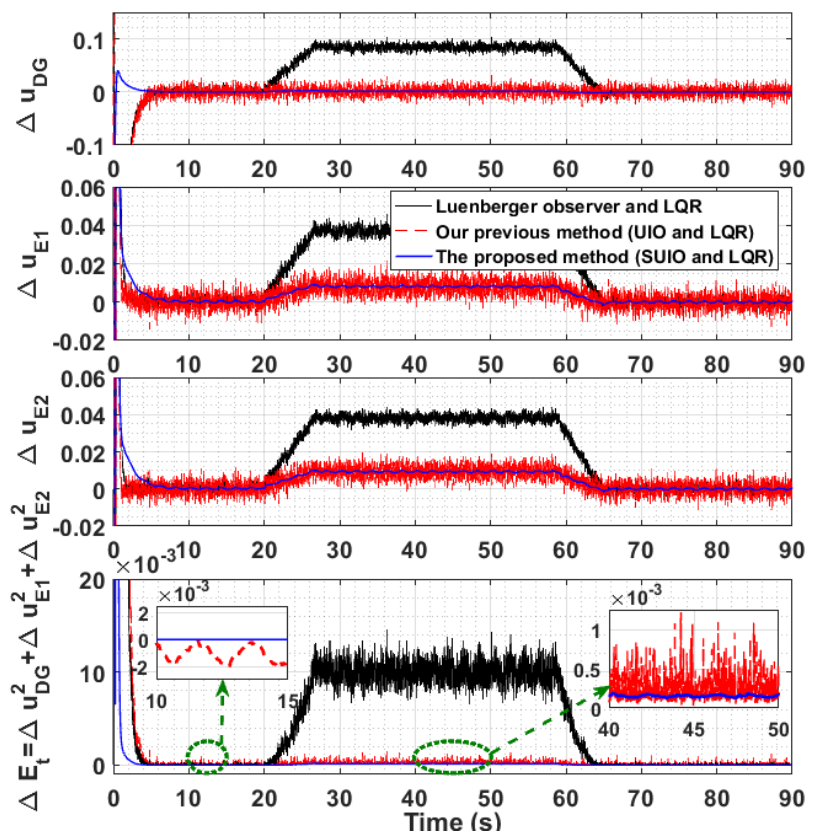

Figure 4.18: Control efforts in case 4

controllers are depicted in Figure 4.17. The control inputs (control efforts) for the DG $\Delta u_{D G}$ and EV stations $\Delta u_{E}$, are shown in Figure 4.18. It is clear that the proposed controller has a significantly better response than the other two controllers. The controller-based on the Luenberger observer does not fully compensate the frequency deviations, and the maximum frequency deviation is 0.0863 p.u.. The maximum frequency deviation is 0.0477 p.u. with SUIO and UIO-based controllers. It can be examined from Figure 4.18 that the Luenberger-based controller requires the most control efforts as compared to other controllers. The total control effort $\Delta E_{t}$ is as shown in the fourth subplot in Figure 4.18. As seen in Figure 4.11, $\Delta E_{t}=$ $1.6179 \mathrm{e}+03$ obtained from SUIO is significantly lower than $2.2581 \mathrm{e}+04$ obtained from UIO and LQR controller [98]. 


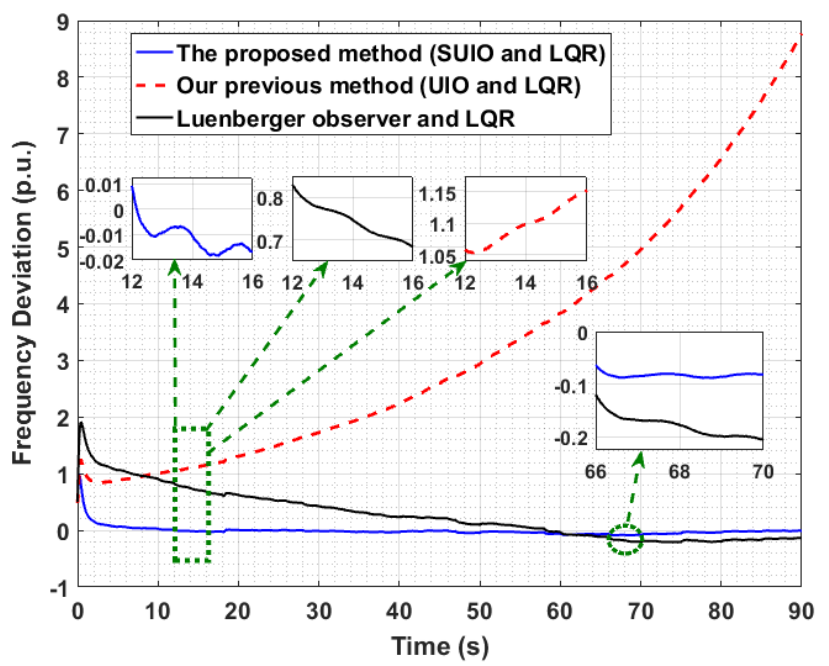

Figure 4.19: Frequency control response in case 5

\section{Case 5}

In this case, both wind power fluctuations and load characteristics in Figure 4.9 are considered, and robustness of the secondary controllers against microgrid parameter changes are evaluated. To replicate the modeling uncertainty, following changes in the parameters are applied: $T_{g}=+25 \% ; T_{d}=-25 \% ; R=+35 \% ; T_{e 1}=-32 \%$; $T_{e 2}=-32 \% ; H_{t}=+20 \%$. Frequency response with secondary control is evaluated for three controllers and is depicted in Figure 4.19. The control inputs (control efforts) $\Delta u_{D G}$ and $\Delta u_{E}$ for the DG and EV stations are shown in Figure 4.20. It is clear that the proposed controller has a significantly better response than Luenberger observer and LQR. Since the SUIO based controller has better performance than UIO based controller to estimate the states and identify the UI, the upper bound value of $L_{a}$ is smaller. Hence, contrary to UIO based control design, SUIO-based controller is satisfied even with having Eigenvalues of $A-B G_{1}$ not far negative (which has a direct impact on control effort). Using UIO based controller in this case causes an unstable control response. Nevertheless, the proposed controller based on SUIO is robust to the parameter changes. Figure 4.20 shows that the UIO-based controller does not have control over the control effort. Also, total power consumption corresponding to the Luenberger-based controller is significantly more than that of the SUIO-based controller.

To quantitatively compare performances of these secondary controllers in terms of control response and estimation accuracy, Integral of Absolute Error (IAE) of frequency deviation, $I A E=\sum_{k=1}^{N S}|\Delta f(k)|$, and Mean Absolute Error between estimated states and real states $M A E=\frac{\sum_{k=1}^{N S}|x(k)-\hat{x}(k)|}{N S}$ are utilized for each controller in Table II. $N S$ is a number of data samples. Table II also shows that the proposed method here outweighs our previous method, Luenberger based and without secondary control in aspects of control performance, control effort, and estimation accuracy. The simulation results in this work specifically show that the proposed SUIO control method outperforms the original UIO control method in [98] since (1) 

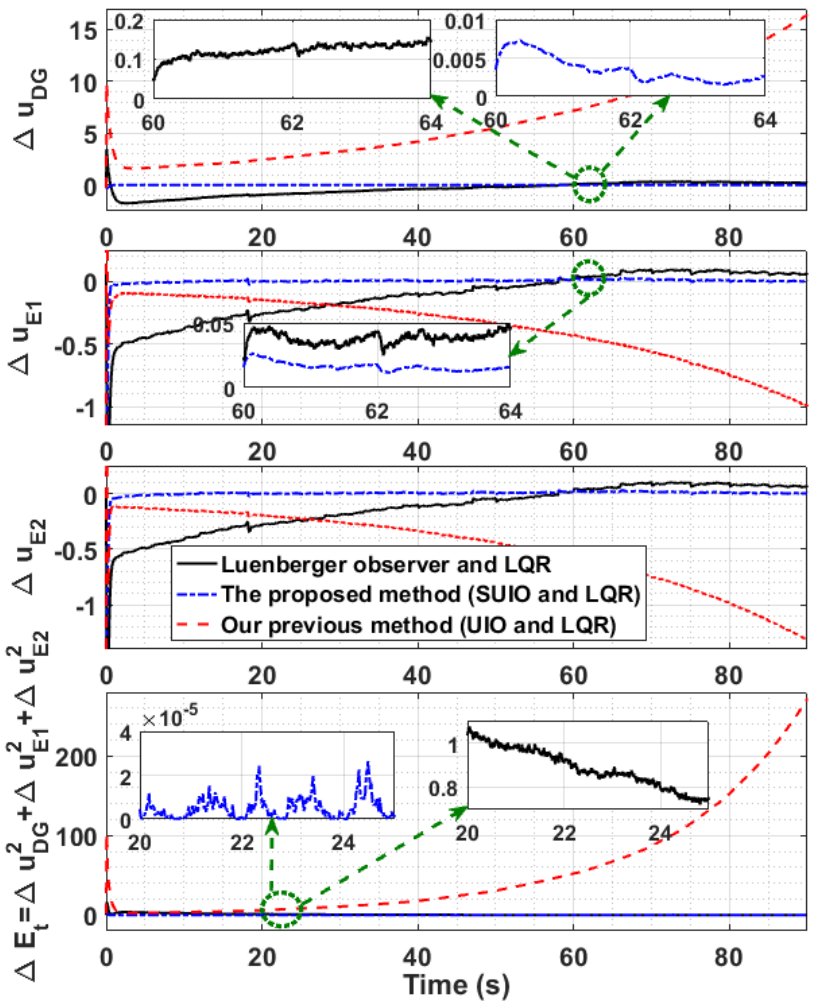

Figure 4.20: Control efforts in case 5

the original UIO is not robust to the microgrid parameter changes. (2) SUIO can very well address the real uncertainties, including renewable energies, load, and measurement noise, compared to the original UIO-based controller because we leverage Kalman Filter for estimating the microgrid states which are not corresponding to uncertainties. (3) SUIO has a more optimal control effort compared to the original $\mathrm{UIO}$ in the control procedure.

\subsubsection{The Proposed Controller Resiliency Under FDI}

This section presents the performance of the proposed resilient control strategy designed under three different scenarios of FDI in the presence of measurement noise and wind power uncertainty shown in Figure 4.9. This proposed SUIO-based controller has compared a joint Luenberger observer and LQR and LQG, which is joint control of Kalman Filter and LQR. Wind power uncertainty as $d_{1}(k)$ and affects only the first system state $x_{1}(k)$. First EVS control input $\Delta u_{E 1}$ that corresponds to the actuator is corrupted by false data injection $d_{2}(k)$.

\section{Scenario 1}

In this scenario, the FDI $d_{2}(k)=\delta x_{4}(k)$ is considered for $k \geq 65$ indicating the adversary mimics the fourth system state, corresponding to the first EVS, with $\delta$ times greater magnitude for a duration of 25 seconds. As shown in Section III, 
Table 4.2: Control and estimation performances of controllers

\begin{tabular}{|c|c|c|c|c|c|}
\hline Cases & Indices & $\begin{array}{l}\text { SUIO } \\
\text { and LQR }\end{array}$ & $\begin{array}{l}\text { UIO } \\
\text { and LQR }\end{array}$ & $\begin{array}{l}\text { Luenberger } \\
\text { and LQR }\end{array}$ & $\begin{array}{l}\text { Without- } \\
\text { Secondary }\end{array}$ \\
\hline \multirow{2}{*}{$\# 1$} & $I A E$ & $1.0951 \mathrm{e}+04$ & $1.1047 \mathrm{e}+04$ & $2.0745 \mathrm{e}+04$ & $2.2592 \mathrm{e}+04$ \\
\hline & $\overline{M A E}$ & 0.0027 & 0.0040 & 0.0125 & \\
\hline \multirow{2}{*}{$\# 2$} & $I A E$ & $7.2192 \mathrm{e}+03$ & $7.2504 \mathrm{e}+03$ & $1.3048 \mathrm{e}+04$ & $1.5357 \mathrm{e}+04$ \\
\hline & $M A E$ & 0.0027 & 0.0041 & 0.0098 & \\
\hline \multirow{2}{*}{$\# \mathbf{3}$} & $I A E$ & $1.2596 \mathrm{e}+04$ & $1.2691 \mathrm{e}+04$ & $2.3492 \mathrm{e}+04$ & $2.6259 \mathrm{e}+04$ \\
\hline & $M A E$ & 0.0027 & 0.0040 & 0.0163 & \\
\hline \multirow{2}{*}{$\# 4$} & $I A E$ & $1.0419 \mathrm{e}+04$ & $1.0617 \mathrm{e}+04$ & $1.8872 \mathrm{e}+04$ & $2.2234 \mathrm{e}+04$ \\
\hline & $M A E$ & 0.0030 & 0.0042 & 0.0132 & \\
\hline \multirow{2}{*}{$\# 5$} & $I A E$ & $1.8790 \mathrm{e}+04$ & $1.4569 \mathrm{e}+06$ & $1.7268 \mathrm{e}+05$ & \\
\hline & $M A E$ & 0.0353 & 2.6771 & 0.4369 & \\
\hline
\end{tabular}

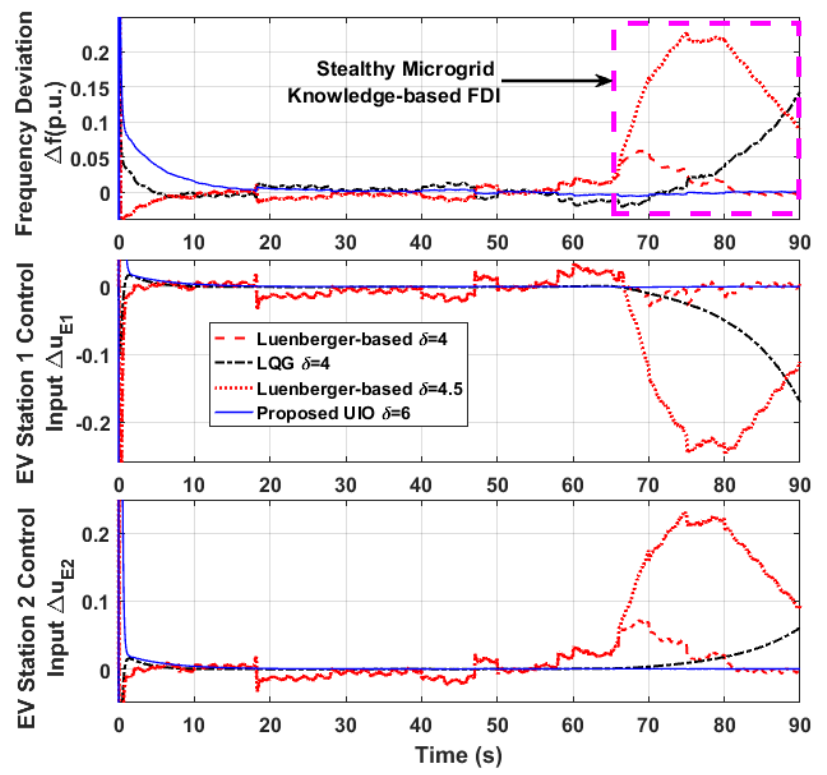

Figure 4.21: Frequency deviation and EVS control inputs in scenario 1

the FDI destabilizes the microgrid equipped with the Luenberger-based and LQG controller, as shown in Figure 4.21. This figure illustrates that LQG and Luenbergerbased controllers get unstable for $\delta \leq 4$ and $\delta \leq 4.5$ respectively although the proposed UIO controller normally operates for $\delta \leq 6$. Figure 4.21 also shows how the control input of the first EVS $\Delta u_{E 1}(k)$ equipped with LQR decreases leading to a decrease in generated power, creating an imbalance in power. The proposed UIO technique is used to detect the wind power uncertainties $d_{1}(k)$, and the FDI $d_{2}(k)$ and a comparison of detected with the actual disturbances is as shown in Figure 4.22 . 


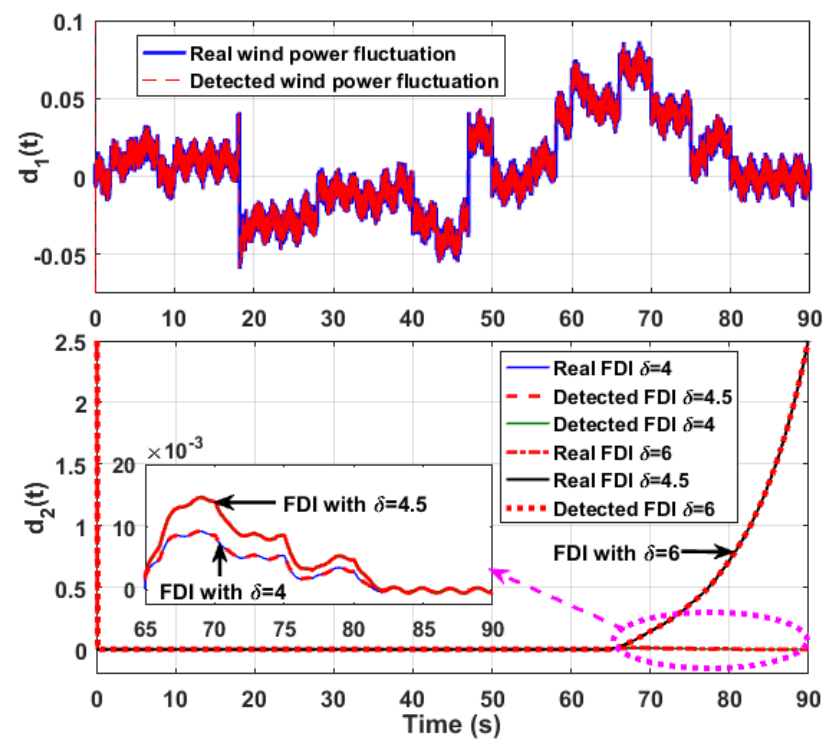

Figure 4.22: Real and detected unknown inputs in scenario 1

\section{Scenario 2}

In this scenario, the FDI consisting of constant pulse $s$ and ramp $r$ disruptions are added to the fourth microgrid state as:

$$
d_{2}(k)= \begin{cases}x_{4}(k)+0.05 s(k) & 30<k \leq 40 \\ x_{4}(k)+0.04 r(k) & k \geq 60\end{cases}
$$

The FDI tremendously degrades frequency control of the microgrid equipped with Luenberger based and LQG controllers as shown in Figure 4.23 where the frequency deviation increases by 0.052 p.u. for Luenberger-based controller and it increases by 0.04 p.u. during disruptive pulse injection. This figure illustrates that the proposed UIO controller maintains frequency stability in spite of Luenberger-based and LQG controllers. Figure 4.23 also shows how the control input of the first EVS $\Delta u_{E 1}(k)$ equipped with Luenbergerger-based and LQG controllers decrease, leading to a decrease in generated power, creating an imbalance in power. The proposed UIO technique is used to detect the FDI $d_{2}(k)$, and a comparison of detected with the actual disturbances is as shown in Figure 4.24.

\section{Scenario 3}

In this scenario, the FDI $d_{2}(k)$ as shown in the equation below, is considered where the adversary injects incorrect data both momentarily and persistently. This data is a function of sinusoids with different magnitudes, phase, and frequency and affects the fourth system state, corresponding to the first EVS. The FDI creates frequency oscillations in the microgrid equipped with standard LQR controller as shown in Figure 4.25 where the frequency drops by $0.07 \mathrm{p} . u$. or $3.5 \mathrm{~Hz}$ for momentary injection and by 0.04 p.u. or $2.0 \mathrm{~Hz}$ for consistent injection. Figure 4.25 also shows the oscillatory response of the control input $\Delta u_{E 1}(k)$ of the first EVS equipped with 


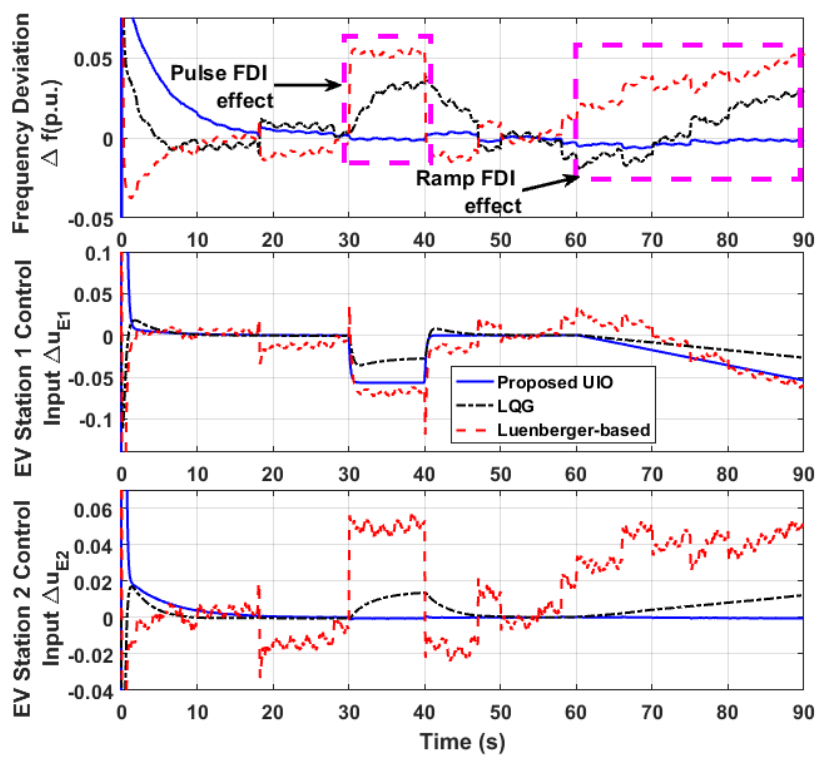

Figure 4.23: Frequency deviation and EVS control inputs in scenario 2

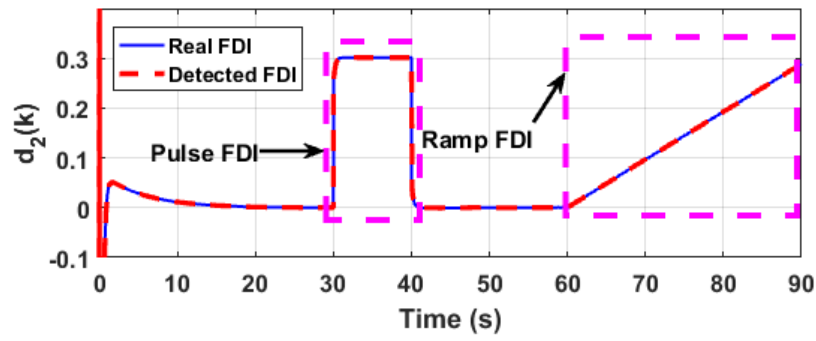

Figure 4.24: Real and detected unknown inputs in scenario 2

LQR. The proposed UIO technique is used to detect the FDI $d_{2}(k)$, and a comparison of detected with the actual disturbances for $d_{2}(k)$ is as shown in Figure 4.26.

$$
d_{2}(k)= \begin{cases}6 \sin (0.5 k+\pi)+2.5 & 30<t \leq 33 \\ \cos (3 k) x_{4}(k)+0.75 \sin (2 k) & 45<t \leq 90\end{cases}
$$

To quantitatively compare performances of these secondary controllers in terms of control response Mean Absolute Error between frequency deviation resulted from utilizing three secondary controllers under FDI and frequency deviation with the secondary controllers without FDI, known as $\Delta f_{0}, M A E=\frac{\sum_{k=1}^{N S}\left|\Delta f(k)-\Delta f_{0}(k)\right|}{N S}$ are utilized for each scenario in Table 4.3. $N S$ is the number of data samples. Table 4.3 also shows that the proposed UIO control method significantly outweighs LQG, Luenberger-based controllers in aspects of control performance. 


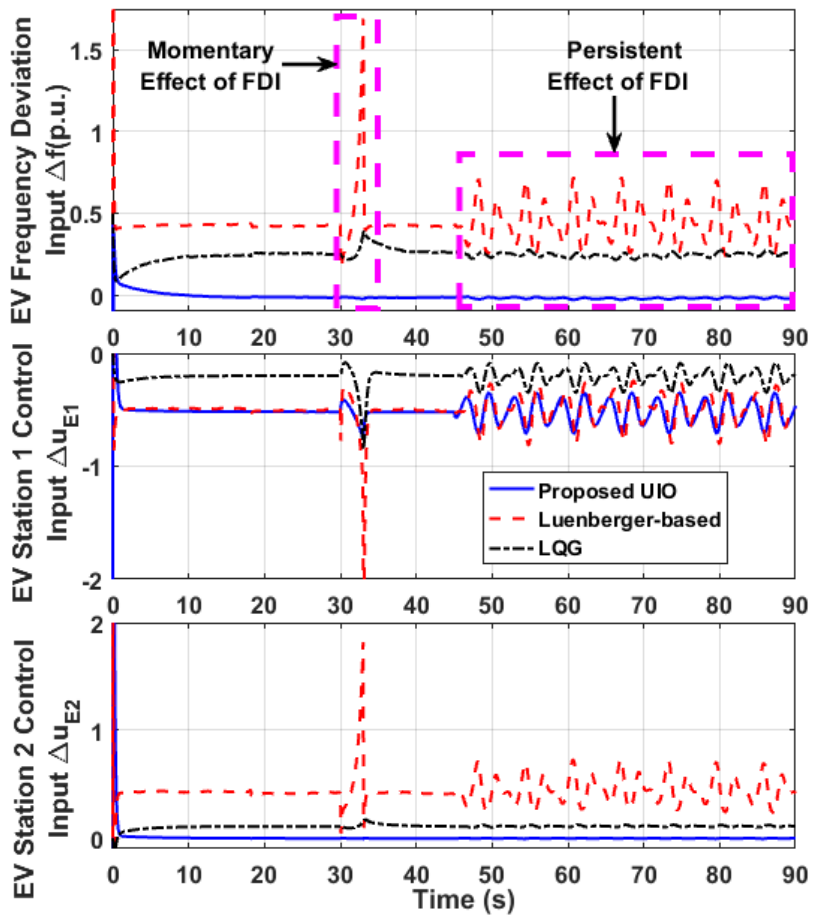

Figure 4.25: Frequency deviation and EVS control inputs in scenario 3

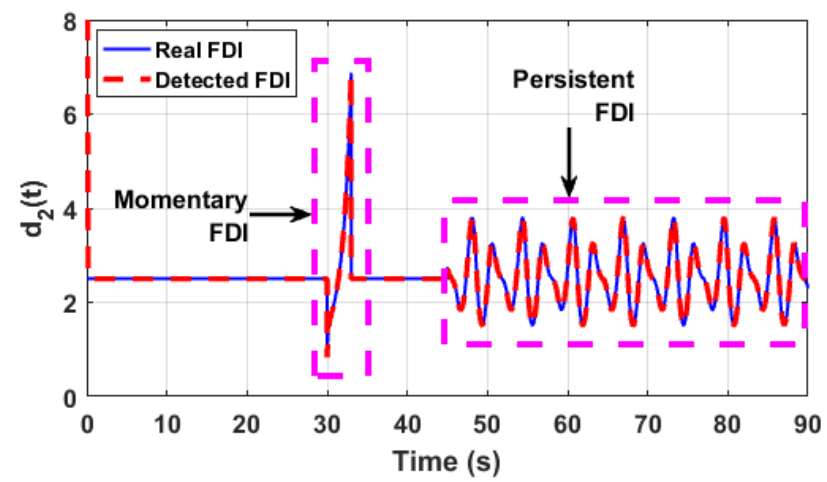

Figure 4.26: Real and detected unknown inputs in scenario 3 Table 4.3: Control performances of controllers

\begin{tabular}{l|l|l|l|l}
\multicolumn{2}{l|}{} & UIO & $\begin{array}{l}\text { Luenberger- } \\
\text { based }\end{array}$ & LQG \\
\hline \multicolumn{2}{l|}{ Scenario 1 } & $5.8438 \mathrm{e}-04$ & 0.0323 & 0.0164 \\
\hline \multirow{2}{*}{ Scenario 2 } & $\delta=4$ & $1.5810 \mathrm{e}-06$ & 0.0190 & 0.0192 \\
\cline { 2 - 5 } & $\delta=4.5$ & $1.9339 \mathrm{e}-06$ & 0.0578 & Unstable \\
\cline { 2 - 5 } & $\delta=6$ & $3.1831 \mathrm{e}-06$ & Unstable & Unstable \\
\hline Scenario 3 & 0.0177 & 0.4340 & 0.2392 \\
\hline
\end{tabular}

\subsubsection{The Proposed Controller Resiliency and scalability Performance Under FDI}

This section implements our proposed UIO-based control technique on the secondary control of an islanded inverter-based microgrid model that was presented earlier 


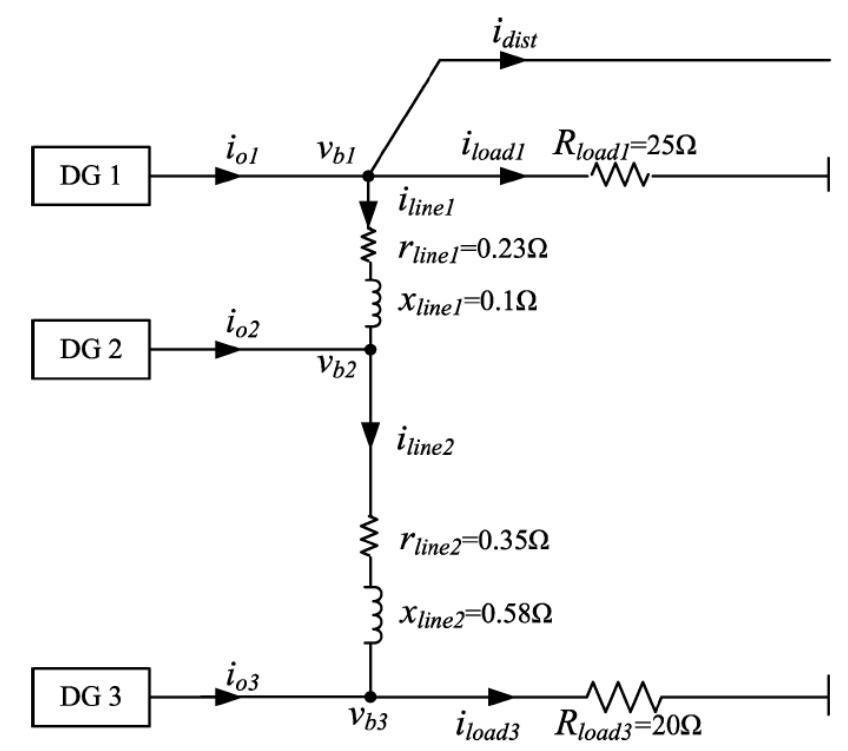

Figure 4.27: The Case Study: Microgrid topology

Table 4.4: Microgrid parameters

\begin{tabular}{l|l|l|l}
\hline \multicolumn{4}{c}{ Inverter Parameters } \\
\hline Parameter & Value & Parameter & Value \\
\hline$f_{s}$ & $8 \mathrm{kHz}$ & $m_{p}$ & $9.4 \mathrm{e}-5$ \\
\hline$L_{f}$ & $1.35 \mathrm{mH}$ & $n_{-} q$ & $1.3 \mathrm{e}-3$ \\
\hline$C_{f}$ & $50 \mu F$ & $K_{p v}$ & 0.05 \\
\hline$r_{f}$ & $0.1 \mathrm{Ohm}$ & $K_{i v}$ & 390 \\
\hline$L_{c}$ & $0.35 \mathrm{mH}$ & $K_{p c}$ & 10.5 \\
\hline$r_{L c}$ & $0.03 \mathrm{Ohm}$ & $K_{i c}$ & $16 \mathrm{e} 3$ \\
\hline$\omega_{c}$ & 31.41 & $F$ & 0.75 \\
\hline
\end{tabular}

[128]. We show that the effect of the false data injected to the inverter angle dynamic of this system is well compensated using our proposed control methodology. In the following, the inverter-based microgrid model is briefly introduced, the secondary control implemented on this model is investigated. Finally the resiliency of the proposed UIO based controller leveraged for this model is examined.

This microgrid model consists of three main modules of inverters, network (line topology), and loads. Inverters dynamics comprises power-sharing controller, output filter, coupling inductor, and current and voltage controller. Each inverter has its own reference frame whose rotation frequency is adjusted by its power sharing controller. This case study is a $220 \mathrm{~V}$ (per phase Root Mean Square), $60 \mathrm{~Hz}$ microgrid equipped with three inverters of equal rating $(10 \mathrm{kVA})$, supplying two loads as shown in Figure 4.27. Figure 4.27 shows some of the microgrid data. These inverters supply two loads connected to the microgrid through lines 1 and 2. More information about the microgrid parameters and initial microgrid conditions are given in Tables 4.4 and 4.5 .

In order to show the efficacy of the proposed UIO based secondary control, in 
Table 4.5: Microgrid initial conditions

\begin{tabular}{|c|c|c|c|}
\hline \multicolumn{4}{|c|}{ Initial Conditions } \\
\hline Parameter & Value & Parameter & Value \\
\hline$V_{\text {od }}$ & {$\left[\begin{array}{llll}380.8 & 381.8 & 380.4\end{array}\right]$} & $V_{o q}$ & {$\left[\begin{array}{lll}0 & 0 & 0\end{array}\right]$} \\
\hline$I_{o d}$ & {$\left[\begin{array}{lll}11.4 & 11.4 & 11.4\end{array}\right]$} & $I_{O q}$ & {$\left[\begin{array}{lll}0.4 & -1.45 & 1.25\end{array}\right]$} \\
\hline$I_{i d}$ & {$\left[\begin{array}{llll}11.4 & 11.4 & 11.4\end{array}\right]$} & $I_{l q}$ & {$[-5.5-7.3-4.6]$} \\
\hline$V_{b d}$ & {$\left[\begin{array}{llll}379.5 & 380.5 & 379\end{array}\right]$} & $V_{b q}$ & {$\left[\begin{array}{lll}-6 & -6 & -5\end{array}\right]$} \\
\hline$\omega_{0}$ & {$[314]$} & $\delta_{0}$ & {$\left[\begin{array}{llll}0 & 1.9 \mathrm{e}-3 & -0.0113\end{array}\right]$} \\
\hline$I_{\text {line1d }}$ & {$[-3.8]$} & $I_{\text {line } 1 q}$ & {$[0.4]$} \\
\hline$I_{\text {line2d }}$ & {$[7.6$} & $I_{\text {line } 2 q}$ & {$[-1.3]$} \\
\hline
\end{tabular}

particular, and secondary control, in general, three simulation cases for this model are shown as follows. In these simulation cases, the secondary control based on our proposed UIO is compared with the secondary control equipped with Linear Quadratic Gaussian (LQG), Luenberger based control, and the microgrid without secondary control (just primary control response).

\section{Case 1}

In this case, the effect of a step load change is investigated. The step load change occurs for load3 at $t=1 \mathrm{~s}$. The angles of inverter 2 and 3 for the microgrid without secondary control and with secondary control using three controllers are depicted in Figures 4.28 and 4.29. As Figures 4.28 and 4.29 displays, the primary control only is not enough to appropriately control microgrid. It is clear that the proposed UIO controller has a significantly better response than the other two controllers. Also, the inverters' frequencies presented in Figures 4.30 shows that the proposed UIO controller compensates the inverters' frequencies better than other secondary controllers.

\section{Case 2}

In this case, the FDI effect to one inverter angle is investigated. The FDI consisting of sinusoid functions is added to the actuator of inverter angle 2 at $t=1.2 \mathrm{~s}$ as:

$$
d(t)=2 \sin (t)+1.25 \sin (0.75 t) \quad t \geq 1.2 s
$$

The angles of inverter 2 and 3 for the microgrid without secondary control and with secondary control equipped with three controllers are depicted in Figures 4.32 and 4.33. It is clear that the proposed UIO controller has a significantly better response than the other two controllers. Also, the inverters' frequencies shown in Figures 4.34 and 4.35 prove that the proposed UIO controller compensates the inverters' frequencies better than other secondary controllers. 


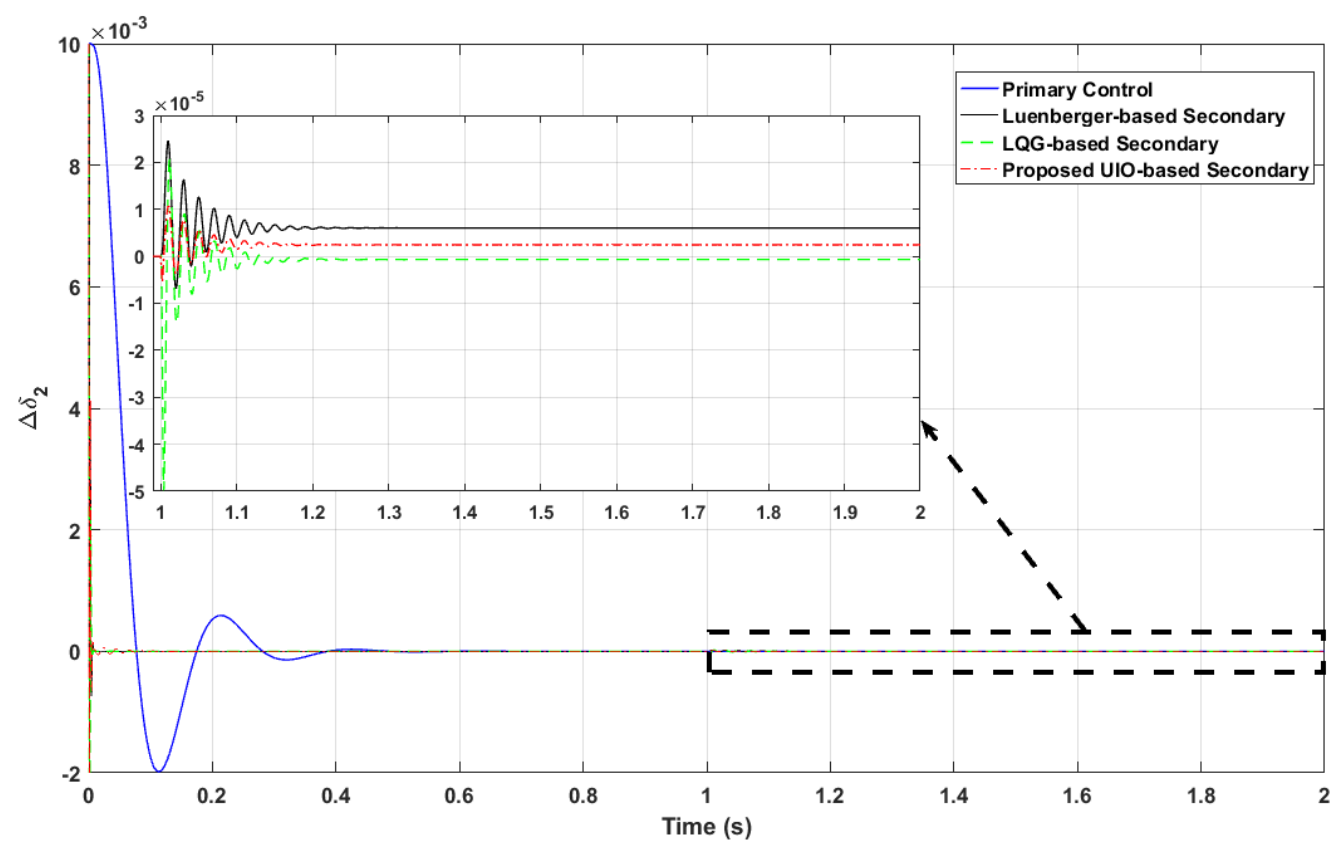

Figure 4.28: Second inverter angle $\delta_{2}$ in scenario 1 (Step load change)

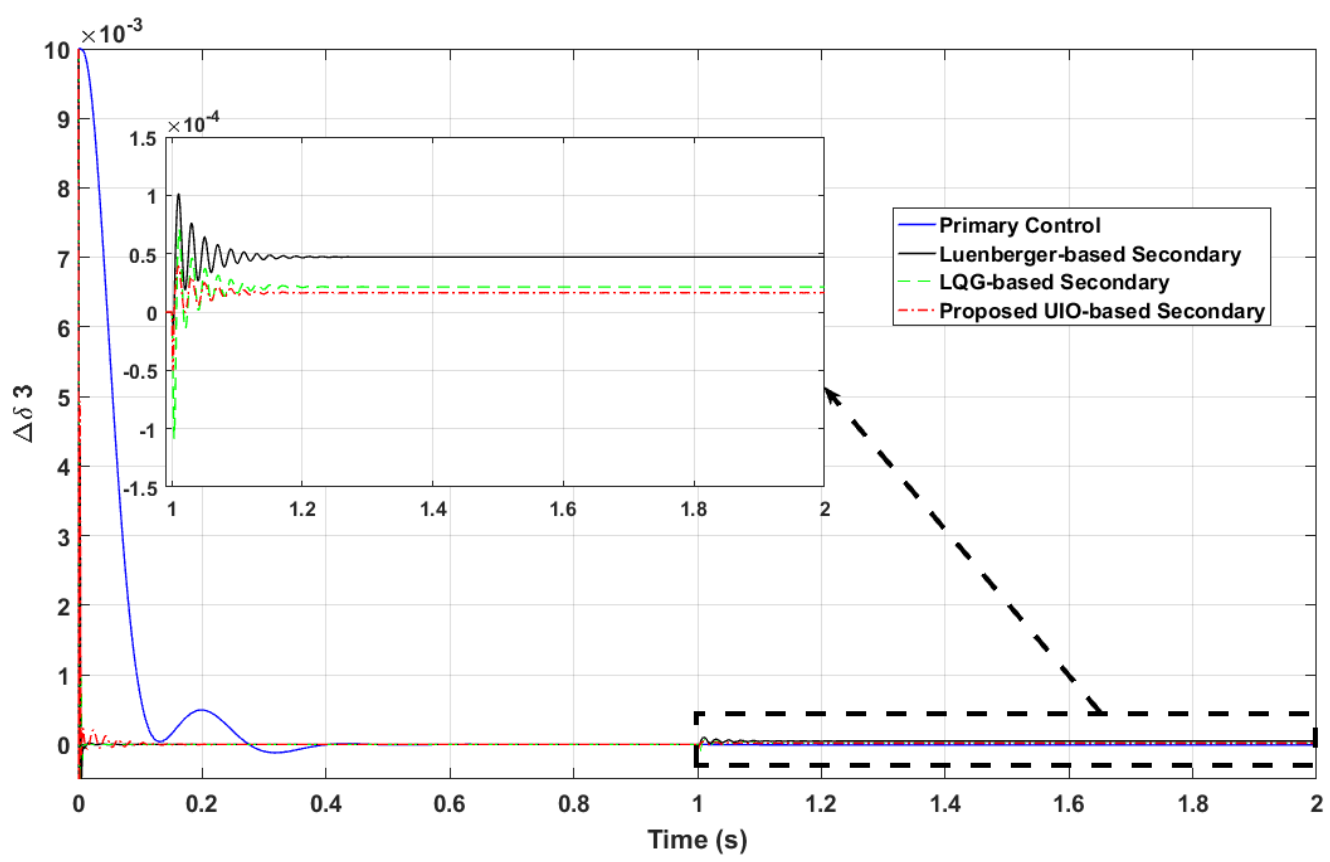

Figure 4.29: Third inverter angle $\delta_{3}$ in scenario 1 (Step load change)

\section{Case 3}

In this case, the stealthy FDI effect to one inverter angle is investigated. As seen in 4.18, all inverter angle dynamics $\Delta \delta_{i}$ are a function of the first inverter active power $\Delta P_{1}$. Thus one destructive FDI can be launched once $\Delta P_{1}$ is stealthily disclosed and used in the data injection (as proved earlier in this chapter). The FDI is added 


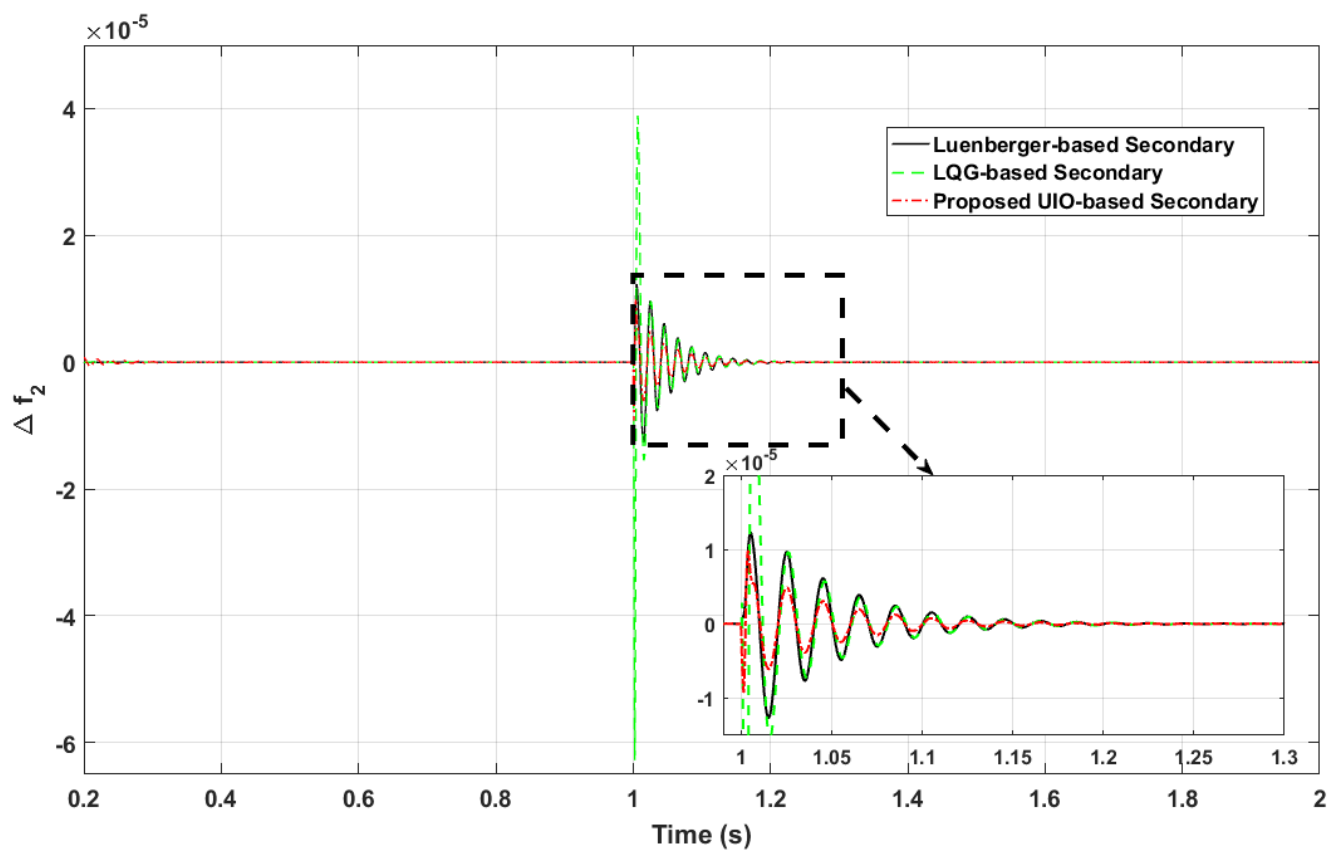

Figure 4.30: Second inverter frequency dynamic $\Delta f_{2}$ in scenario 1 (Step load change)

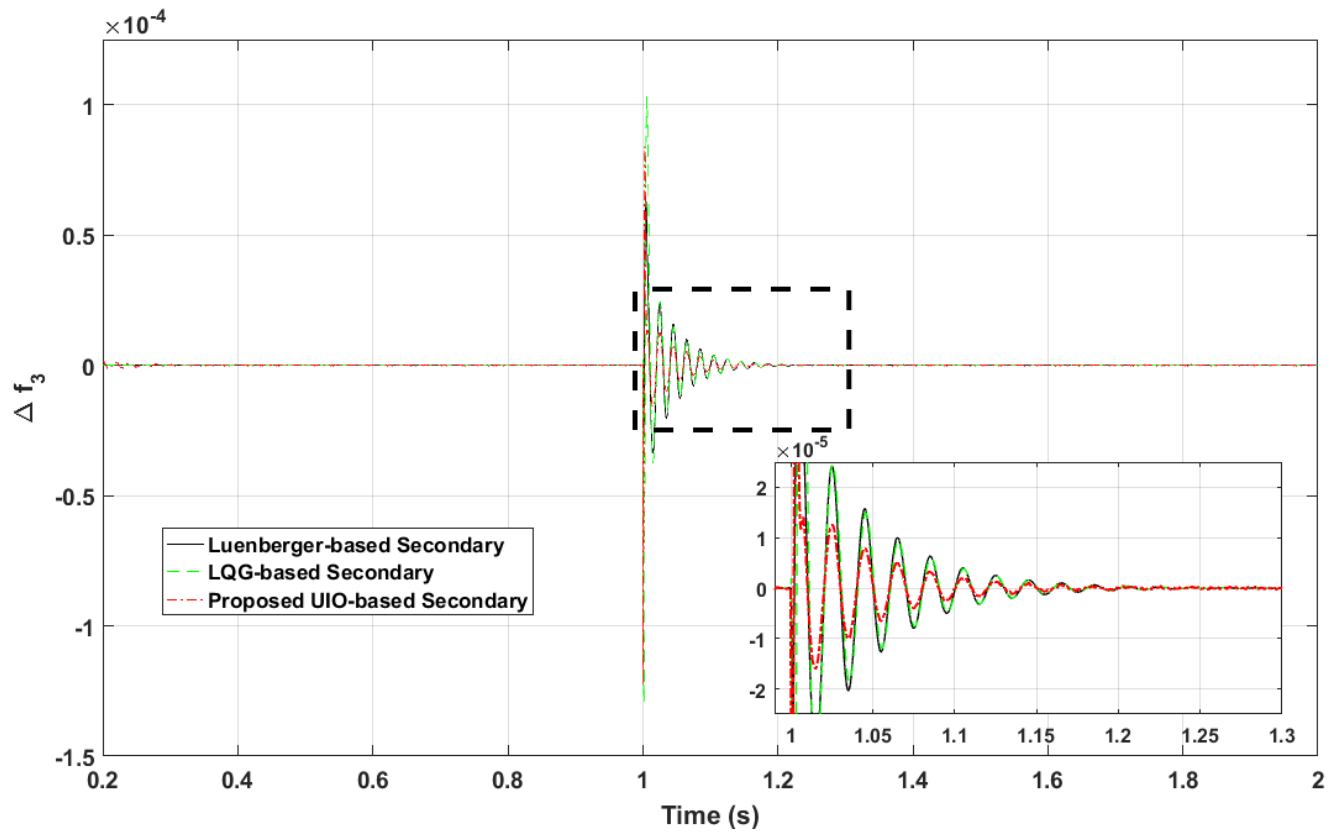

Figure 4.31: Third inverter frequency dynamic $\Delta f_{3}$ in scenario 1 (Step load change)

to the actuator of inverter angle 2 at $t=1.4 \mathrm{~s}$ as:

$$
d(t)=0.5 \Delta P_{1}(t) \quad t \geq 1.4 s
$$

The angles of inverter 2 and 3 for the microgrid without secondary control and with secondary control equipped with three controllers are depicted in Figures 4.36 and 


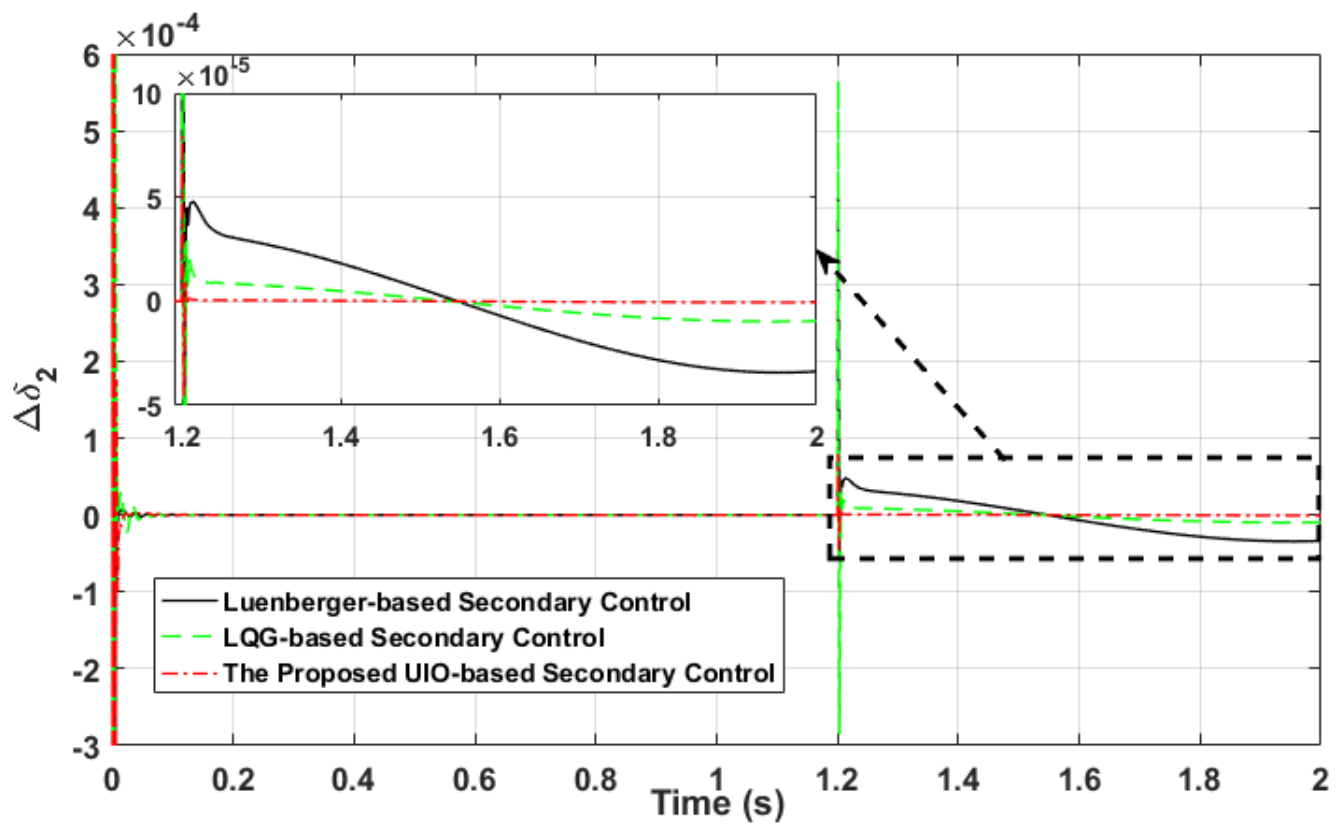

Figure 4.32: Second inverter angle $\delta_{2}$ in scenario 2 (FDI to the second inverter's actuator)

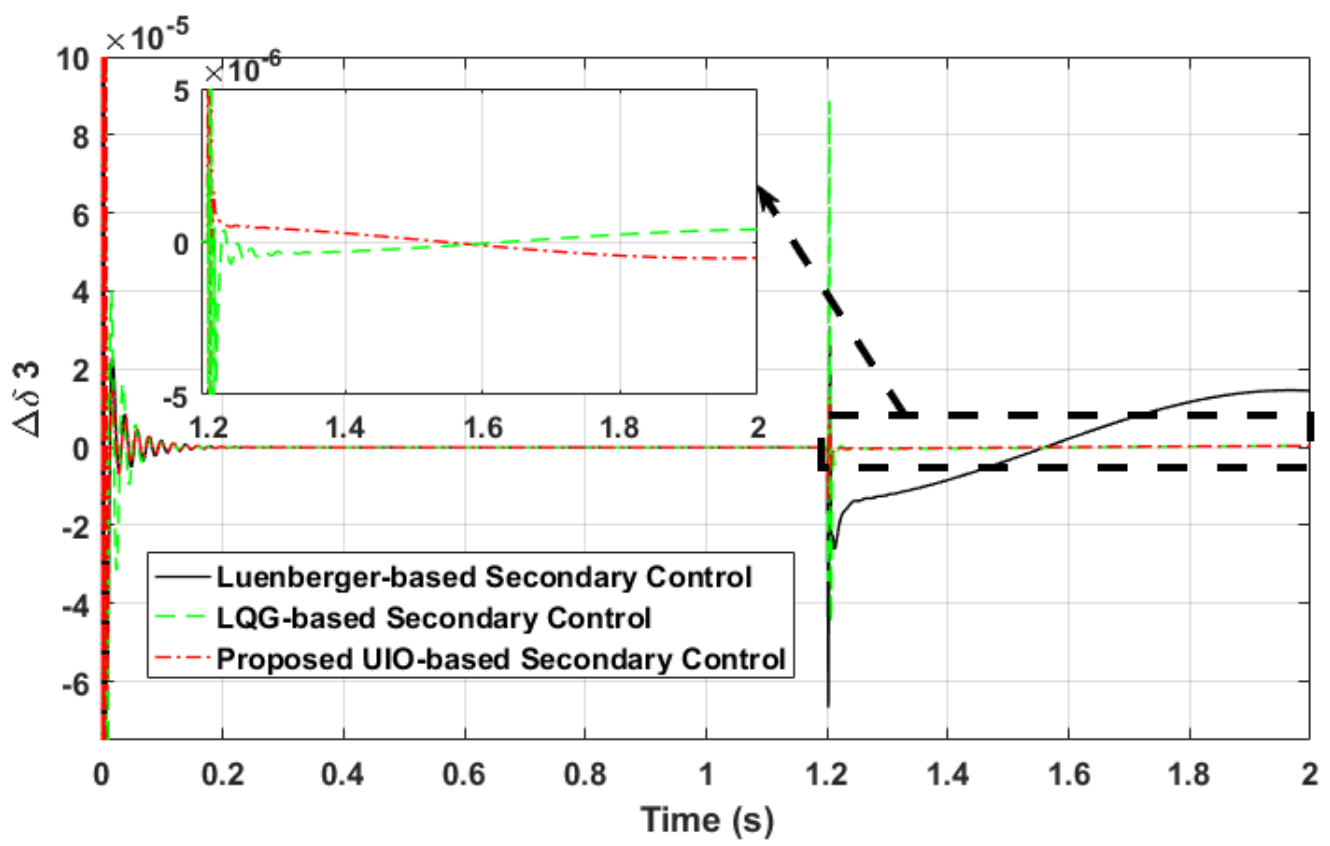

Figure 4.33: Third inverter angle $\delta_{3}$ in scenario 2 (FDI to the second inverter's actuator)

4.37. It is clear that the proposed UIO controller can still resiliently control the microgrid; however, this stealthy FDI can destabilize the microgrid equipped with Luenberger and LQG based secondary control. The Luenberger-based secondary controller operates significantly more unstable compared to the LQG-based con- 


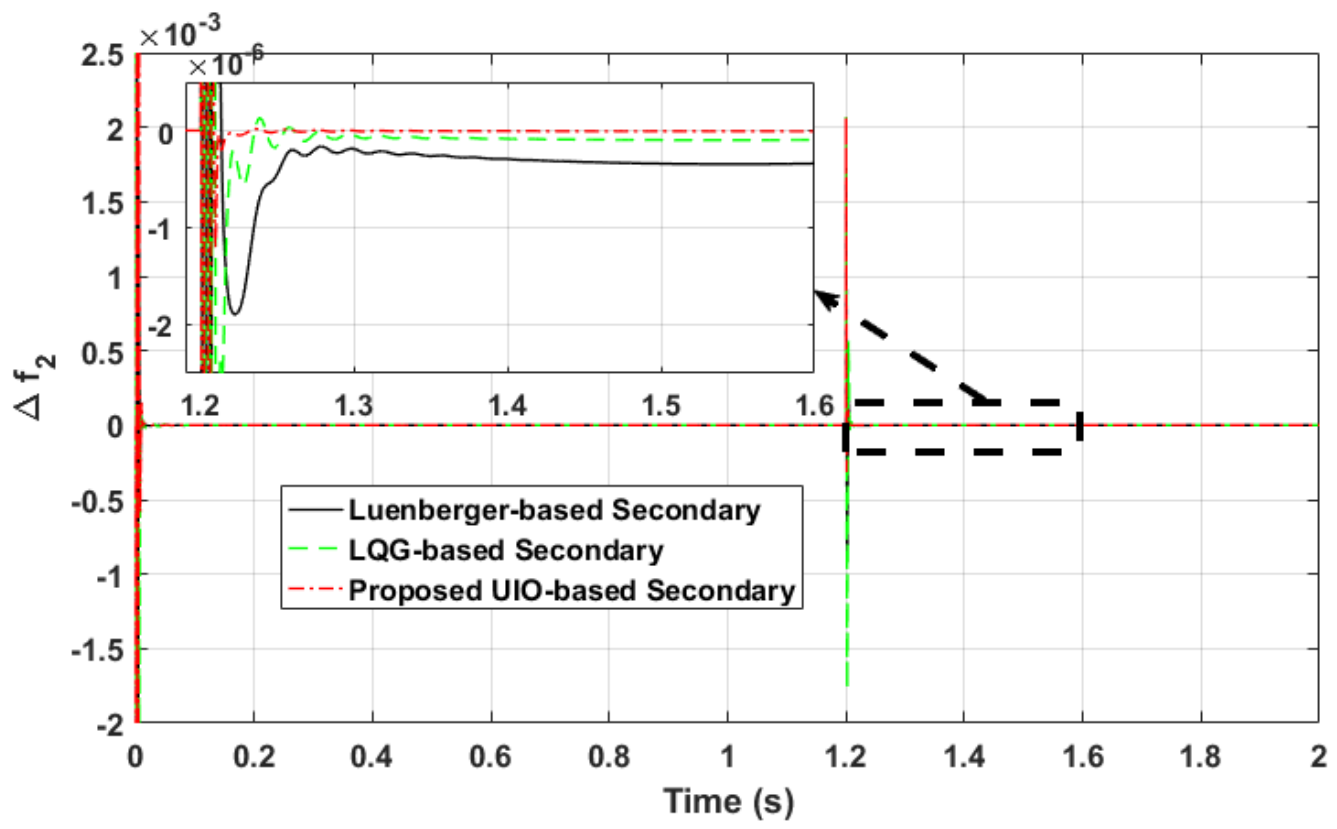

Figure 4.34: Second inverter frequency dynamic $\Delta f_{2}$ in scenario 2 (FDI to the second inverter's actuator)

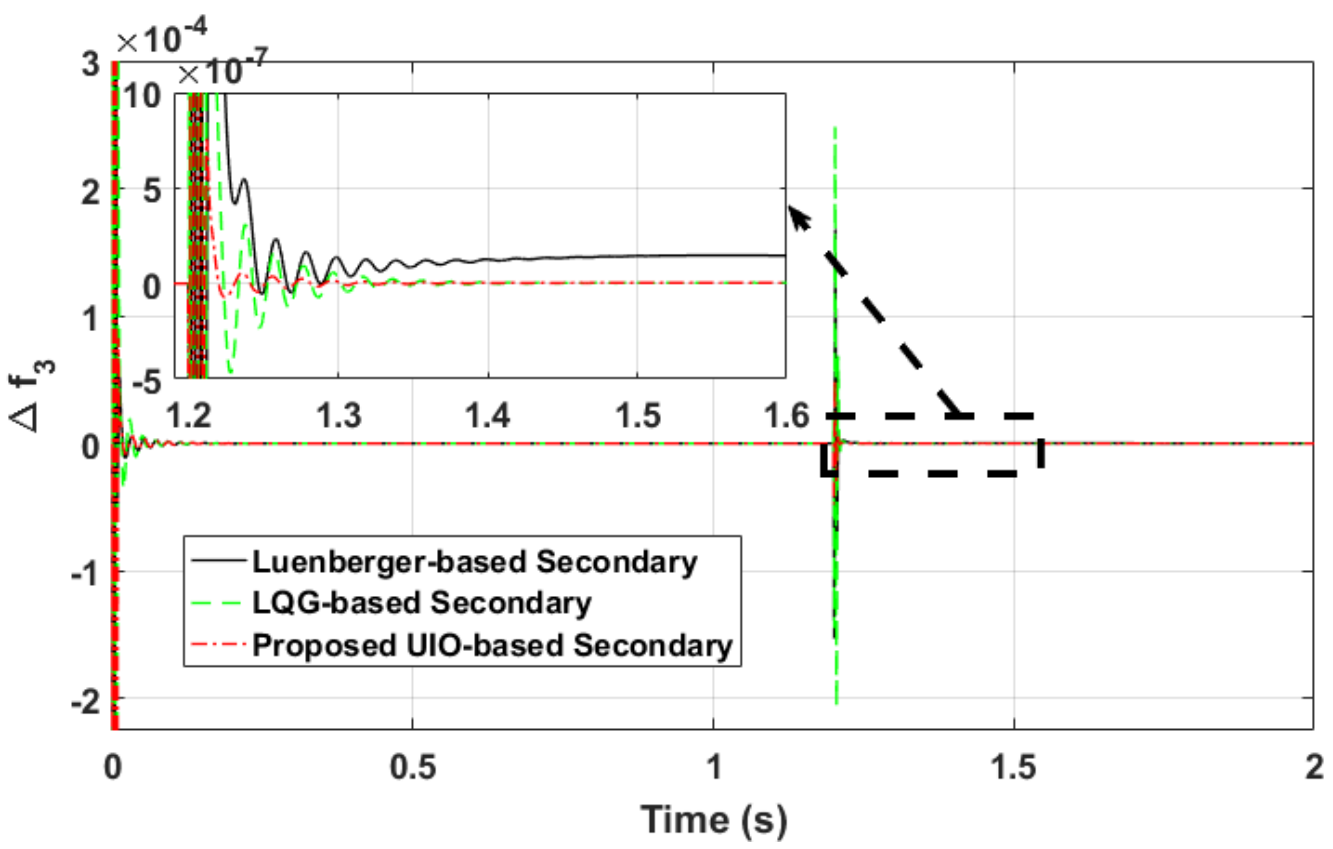

Figure 4.35: Third inverter frequency dynamic $\Delta f_{3}$ in scenario 2 (FDI to the second inverter's actuator)

troller. Also, the inverters' frequencies shown in Figures 4.38 and 4.39 prove that the proposed UIO controller, unlike the other secondary controllers, compensates the inverters' frequencies. In fact, this simulation case verifies that the proposed UIO-based controller properly maintains the microgrid resiliency. 


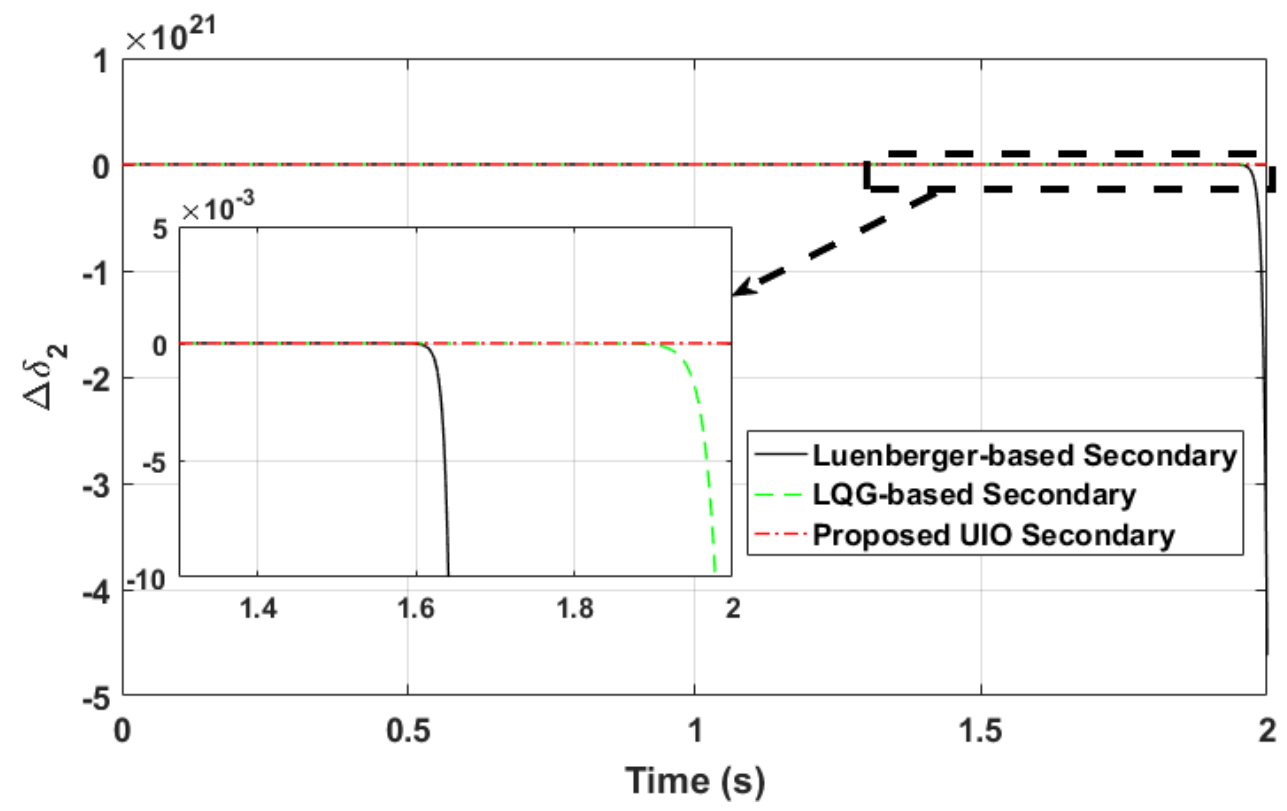

Figure 4.36: Second inverter angle $\delta_{2}$ in scenario 3 (Stealthy FDI to the second inverter's actuator)

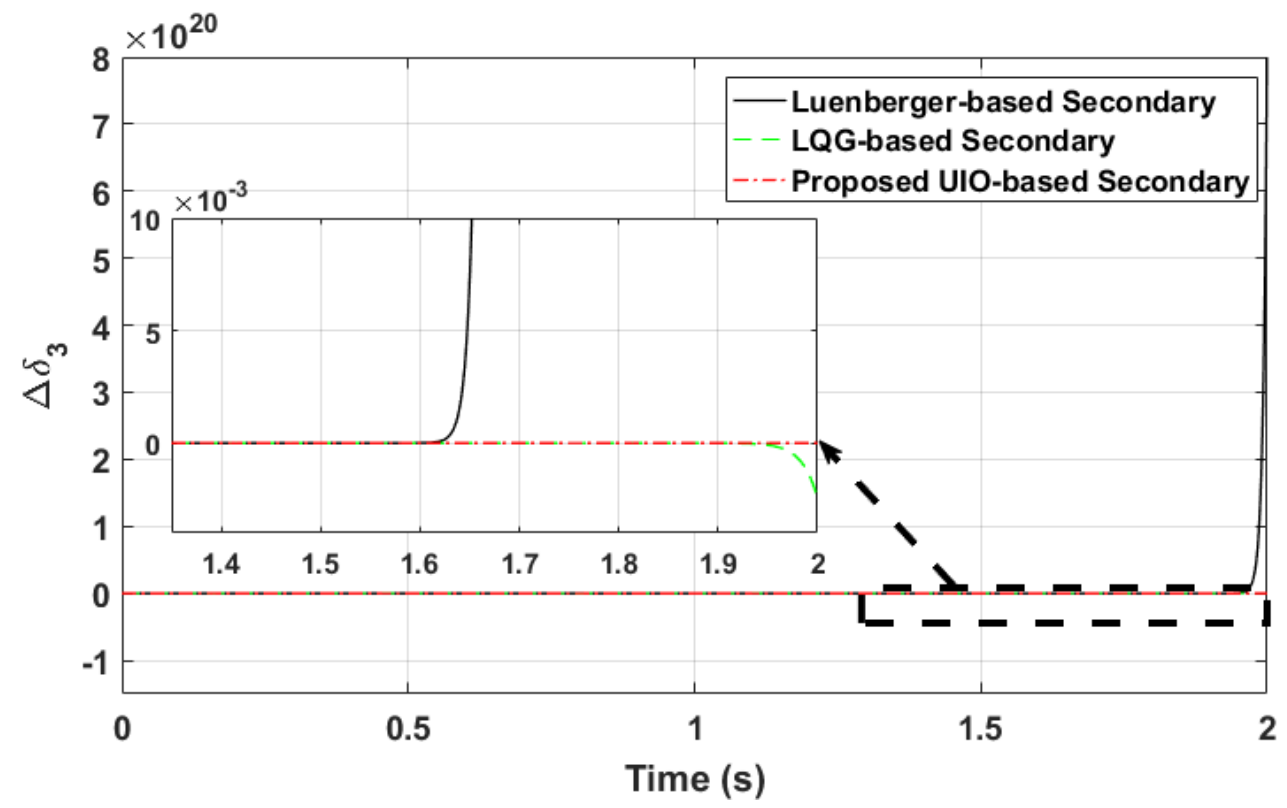

Figure 4.37: Third inverter angle $\delta_{3}$ in scenario 3 (Stealthy FDI to the second inverter's actuator)

\subsection{Conclusion}

This section focuses on control of microgrid in the presence of renewable and load uncertainties as well as cyber manipulation effect on the microgrid actuators. At first, vulnerability analysis verifies that an intelligent, stealthy FDI can severely 


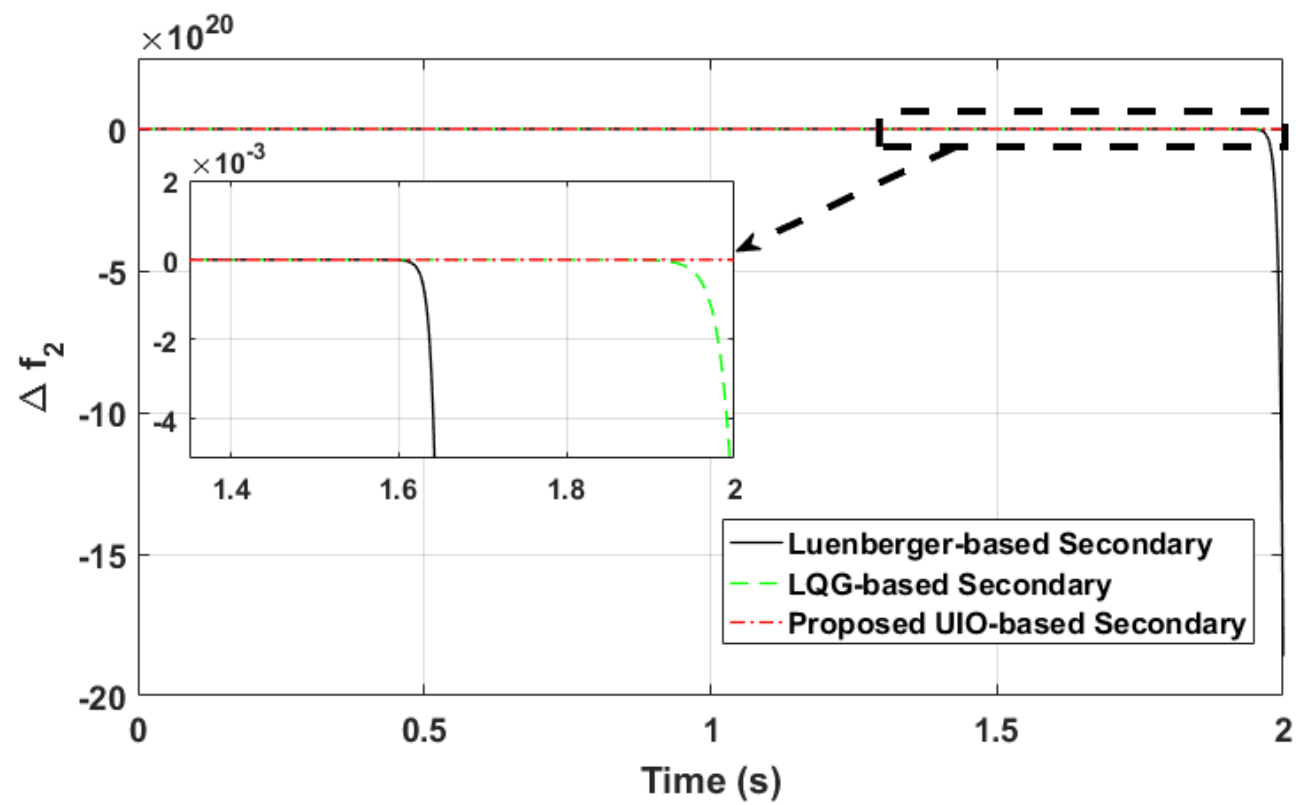

Figure 4.38: Second inverter frequency dynamic $\Delta f_{2}$ in scenario 3 (Stealthy FDI to the second inverter's actuator)

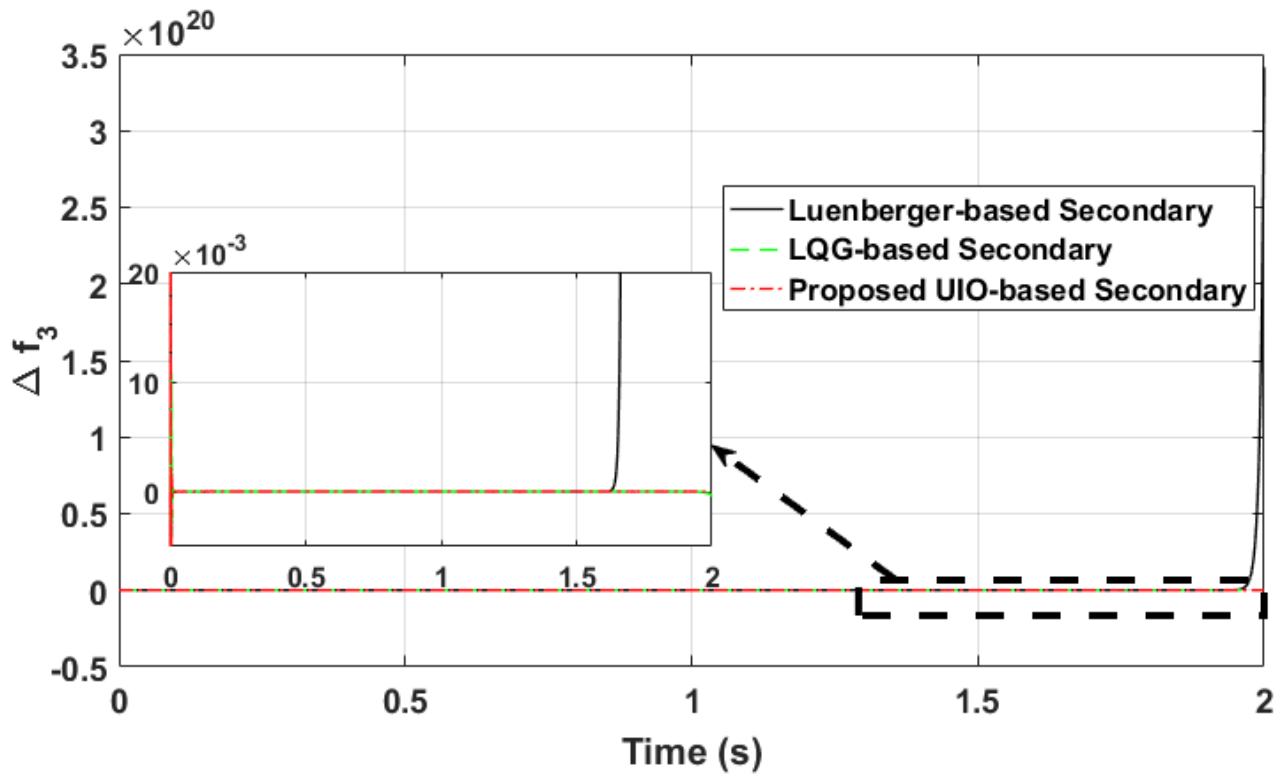

Figure 4.39: Third inverter frequency dynamic $\Delta f_{3}$ in scenario 3 (Stealthy FDI to the second inverter's actuator)

jeopardize the stability of microgrid, especially under the knowledge of real-data of control inputs. Therefore, an optimal resilient controller based on a joint UIO and LQR is proposed. This proposed two-layer control algorithm includes UI detection, microgrid state estimation, and frequency deviation mitigation for the microgrid equipped with electric vehicle charging stations and one inverter-based microgrid 
model. The simulation results verify the significant resilient and optimal performance of the proposed strategy compared to that of optimal controllers based on Luenberger observer and LQG. 


\section{Chapter 5}

\section{Conclusion}

\subsection{Concluding Remarks}

The research work presented in this dissertation has focused on three main microgrids challenges of EV and renewable energy connection and cyber security. An account of the main contributions of this dissertation is given as follows:

1. Chapter 2 solves two important problems associated with EV integrated to microgrids. In the first problem, a combined AI-based optimization algorithm and stochastic modeling approach are leveraged to find an optimal EV charging and discharging schedule such that the daily load profile is flattened and in turn, the energy cost is minimized. The stochastic modeling approach is applied to consider uncertainties from EV travel throughout the microgrid, which is IEEE 13-node feeder system. Particle Swarm Optimization (PSO) algorithm is used to minimize the objective function regarding flattening daily load profile (peak load shaving and valley load filling) and consider the technical constraints, e.g., EV batteries' State-of-Charging. In the second problem, a multi-objective optimization based on PSO is developed to investigate the best locations for EV parking-lots connected to the microgrid such that the microgrid has minimum power loss and voltage unbalance. It is shown that this multi-objective algorithm outweighs the single and standard optimization algorithms used for this problem. This chapter merely focuses on static analyses of EV stations affecting microgrid, including voltage drop and power loss, not the associated dynamic analyses, e.g., EV stations participation into control frequency, which is taken into account in the next chapter.

2. Chapter 3 introduces two AI-based controllers, named human brain emotional learning and Hebb learning controllers, to control frequency in microgrids equipped with EV stations. These two controllers are applied to properly actuate energy sources in the microgrid, like diesel generators, and EV stations, and mitigate frequency deviation resulted from uncertainties and perturbations of renewables and load changes. These two controllers were inspired by the human decision-making process and artificial neural networks respectively. These 
controllers do not require the exact mathematical microgrid model for their control procedures, which is a great advantage for power systems with high penetration of uncertainties. In addition to their model-free operation, these controllers perform as self-tuning Proportional-Integral-Derivative (PID) regulators which appropriately respond to uncertainties and intermittencies, e.g., renewable energies and load variation, in a real-time manner. It means that these two controllers do not need to be trained similar to normal neural network techniques. The other advantage of these controllers is that they do not require microgrid information measured from the system. However, these controllers cannot optimally perform to compensate frequency deviation since they do not have enough information from the microgrid model and measured information. Furthermore, these controllers can be implemented for small systems. Hence, one analytic control approach is developed in the next chapter to overcome the weaknesses of these AI-based controllers.

3. Chapter 4 develops an analytical control method based on Unknown Input Observer (UIO) which is robust against uncertainties. Since this method adopts the measured information from the microgrid through communication links to optimally operate the microgrid, it is prone to cyber disruptions that can deteriorate the control performance and even cause instability. In this chapter, a destructive and disruptive type of cyber anomaly is investigated. This cyber disruption mainly targets the control actuators of the microgrid by manipulating the control packets sent from the controller to the energy source actuators, i.e., this cyber anomaly occurs once the energy supplies of microgrid receive false data from the control center, so-called False Data Injection (FDI) to control actuators. Our vulnerability analysis proves that this FDI can be more detrimental once the microgrid flowing information is disclosed and used in the FDI. Hence we show that the UIO based controller resiliently works under these cyber manipulation also. To prove the scalability of this controller, we applied this controller for an inverter-based microgrid model which have larger state-space representation. The simulation results show the efficacy of this controller in terms of uncertainties robustness, cyber disruption resiliency, and scalable capability.

\subsection{Future Work}

Based on the outcomes of this dissertation, several extensions to this work are identified to be continued in the future. These are briefly discussed as follows:

- Many challenges are not still addressed regarding EV integration into microgrids. The first challenge is the systematic and external uncertainties arising from the system model, user behavior and coordination approach. Due to the high penetration of renewable energies in microgrids, the necessity of considering the existence of these type of uncertainties along with load uncertainties is essential to perfectly model the grid. Furthermore, most of the available 
methods for EV charging assume that the customers are rational and they have perfect knowledge about the system parameters and hence use stochastic methodologies for behavior modeling. An accurate stochastic model to handle uncertainties of EV users' behaviors and mobility profile including arrival and departure time is difficult due to privacy and individual economic concerns of customers. Most of the optimization methodologies, including our method, applied in this problem do not work in an online or real-time frame. The third challenge is associated with how communications occur as centralized or decentralized. In the centralized control methods, all EVs in the system is usually controlled by a central system operator. Centralized control methods may not be practical due to: 1) the lack of global information of charging demand of EVs; 2) the computation difficulties with potentially increasing population of EVs; and 3) the reluctance of consumers to abandon the decision-making authority regarding the charging of their EVs. In Decentralized control methods, each EV usually determines its charging profile based on the local or global information. Reinforcement learning techniques can be leveraged to optimize charging and discharging schedule of EVs in real-time. The reinforcement learning part of artificial intelligence-based techniques have enabled much better solutions to large-scale sequential decision problems. Since distributed implementations are more practical, a distributed reinforcement learning methodology can be utilized for optimal charging profile of individual charging stations and broadcasting the local results to the other neighboring stations.

- Many known cyber disruptions are not studied here and can still distort or cripple microgrid control procedure, such as time-delay and Denial-of-Service (DoS). Using FDI and time-delay attack, adversaries can induce oscillatory responses in a microgrid identical to real oscillation events in power systems. In these cases, the microgrid controllers, especially those, which are based on data-driven methods, may trigger some harsh remedial actions, such as load shedding or islanding, by mistake. These actions cause huge financial loss for both utility and customers. Also, DoS can target the communication links in sensing loops of microgrids and make the exchanged data pockets temporarily unavailable, which causes unstable microgrid operation. Thus it is required to design a resilient controller based on state-estimations and/or artificial intelligence techniques to address these potential issues in communication channels.

- The inverter-based microgrid model used in this dissertation can be developed to comprehensively and accurately model a microgrid. This model should cover three-phase and unbalance characteristics of distribution grids. In order to utilize a more accurate model of microgrid, non-linear system model can be used. In this case, we do not need linear assumptions, e.g. linearizing the system around a specific operating point, etc. It should be noted that there are many papers focused on secondary control of this inverter-based microgrid model. We can work on proposing a distributed control for this 
microgrid which is resilient against cyber disruptions and computationally efficient method that facilitates its application for larger-scale microgrids. 


\section{Bibliography}

[1] "10 Years after the 2003 Northeast Blackout," https://www.energy.gov/oe/articles/10-years-after-2003-northeast-blackout, accessed: 2013-08-14.

[2] A. Hirsch, Y. Parag, and J. Guerrero, "Microgrids: A review of technologies, key drivers, and outstanding issues," Renewable and Sustainable Energy Reviews, vol. 90, pp. 402-411, 2018.

[3] D. T. Ton and M. A. Smith, "The u.s. department of energy's microgrid initiative," The Electricity Journal, vol. 25, pp. 84-94, 2012.

[4] T. N. Pham, H. Trinh et al., "Load frequency control of power systems with electric vehicles and diverse transmission links using distributed functional observers," IEEE Transactions on Smart Grid, vol. 7, no. 1, pp. 238-252, 2016.

[5] M.-H. Khooban, T. Niknam, M. Shasadeghi, T. Dragicevic, and F. Blaabjerg, "Load frequency control in microgrids based on a stochastic noninteger controller," IEEE Transactions on Sustainable Energy, vol. 9, no. 2, pp. 853-861, 2018.

[6] T. I. E. Agency, "Global ev outlook 2017," Electric Vehicles, vol. 3, pp. 1-71, 2017.

[7] T. N. Pham, S. Nahavandi, H. Trinh, and K. P. Wong, "Static output feedback frequency stabilization of time-delay power systems with coordinated electric vehicles state of charge control," IEEE Transactions on Power Systems, vol. 32, no. 5, pp. 3862-3874, 2017.

[8] J. Pahasa and I. Ngamroo, "Coordinated control of wind turbine blade pitch angle and phevs using mpcs for load frequency control of microgrid." IEEE Systems Journal, vol. 10, no. 1, pp. 97-105, 2016.

[9] A. Abdallah and X. S. Shen, "Lightweight authentication and privacypreserving scheme for v2g connections," IEEE Transactions on Vehicular Technology, vol. 66, no. 3, pp. 2615-2629, 2017.

[10] Z. Ma, D. Callaway, and I. Hiskens, "Decentralized charging control for large populations of plug-in electric vehicles: Application of the nash certainty 
equivalence principle," in 2010 IEEE International Conference on Control Applications, 09 2010, pp. 191-195.

[11] Y. He, B. Venkatesh, and L. Guan, "Optimal scheduling for charging and discharging of electric vehicles," IEEE Transactions on Smart Grid, vol. 3, no. 3, pp. 1095-1105, 092012.

[12] H. T. M. M. E.-K. M. H. Rehmani, "Electric vehicle scheduling and charging in smart cities," in Transportation and Power Grid in Smart Cities: Communication Networks and Services. Wiley, 2019, pp. 1-688.

[13] M.-H. Khooban, T. Niknam, F. Blaabjerg, P. Davari, and T. Dragicevic, "A robust adaptive load frequency control for micro-grids," ISA Transactions, vol. 65, pp. 220 - 229, 2016. [Online]. Available: http://www.sciencedirect.com/science/article/pii/S001905781630146X

[14] A. Safari, F. Babaei, and M. Farrokhifar, "A load frequency control using a pso-based ann for micro-grids in the presence of electric vehicles," International Journal of Ambient Energy, vol. 0, no. 0, pp. 1-13, 2019. [Online]. Available: https://doi.org/10.1080/01430750.2018.1563811

[15] M.-H. Khooban, "Secondary load frequency control of time-delay stand-alone microgrids with electric vehicles," IEEE Transactions on Industrial Electronics, vol. 65, no. 9, pp. 7416-7422, 2018.

[16] I. Stellios, P. Kotzanikolaou, M. Psarakis, C. Alcaraz, and J. Lopez, "A survey of iot-enabled cyberattacks: Assessing attack paths to critical infrastructures and services," IEEE Communications Surveys Tutorials, vol. 20, no. 4, pp. 3453-3495, 082018.

[17] J. W. Simpson-Porco, Q. Shafiee, F. Dörfler, J. C. Vasquez, J. M. Guerrero, and F. Bullo, "Secondary frequency and voltage control of islanded microgrids via distributed averaging," IEEE Transactions on Industrial Electronics, vol. 62, no. 11, pp. 7025-7038, 112015.

[18] C. Ahumada, R. Cárdenas, D. Saez, and J. M. Guerrero, "Secondary control strategies for frequency restoration in islanded microgrids with consideration of communication delays," IEEE Transactions on Smart Grid, vol. 7, no. 3, pp. 1430-1441, 2016.

[19] A. Khalil, Z. Rajab, A. Alfergani, and O. Mohamed, "The impact of the time delay on the load frequency control system in microgrid with plug-in-electric vehicles," Sustainable Cities and Society, vol. 35, pp. 365 - 377, 2017. [Online]. Available: http://www.sciencedirect.com/science/article/pii/S2210670717306819

[20] https://en.wikipedia.org/wiki/Plug-in-electric-vehicles-in-the-United-States.

[21] www.mckinsey.com/. 
[22] M. H. Bioki, M. Z. Jahromi, and M. Rashidinejad, "A combinatorial artificial intelligence real-time solution to the unit commitment problem incorporating v2g," Electrical Engineering, vol. 95, no. 4, pp. 341-355, 2013.

[23] M. E. Khodayar, S. D. Manshadi, H. Wu, and J. Lin, "Multiple period ramping processes in day-ahead electricity markets," IEEE Transactions on Sustainable Energy, vol. 7, no. 4, pp. 1634-1645, 2016.

[24] M. R. Khalghani, M. Ramezani, and M. Rajabi-Mashhadi, "Demonstrating the importance of applying a new probabilistic power flow strategy to evaluate power systems with high penetration of wind farms," Journal of Energy Engineering, vol. 142, no. 4, p. 04016002, 2016.

[25] R.-C. Leou, C.-L. Su, and C.-N. Lu, "Stochastic analyses of electric vehicle charging impacts on distribution network," IEEE Transactions on Power Systems, vol. 29, no. 3, pp. 1055-1063, 2014.

[26] C. Chen and S. Duan, "Optimal integration of plug-in hybrid electric vehicles in microgrids," IEEE Transactions on Industrial Informatics, vol. 10, no. 3, pp. 1917-1926, 2014.

[27] W. Leterme, F. Ruelens, B. Claessens, and R. Belmans, "A flexible stochastic optimization method for wind power balancing with phevs," IEEE Transactions on Smart Grid, vol. 5, no. 3, pp. 1238-1245, 2014.

[28] S. D. Manshadi and M. E. Khodayar, "A hierarchical electricity market structure for the smart grid paradigm," IEEE Transactions on Smart Grid, vol. 7, no. 4, pp. 1866-1875, 2016.

[29] M. F. Astaneh, B. P. Bhattarai, B. Bak-Jensen, W. Hu, J. R. Pillai, and Z. Chen, "A novel technique to enhance demand responsiveness: An ev based test case," in 2015 IEEE PES Asia-Pacific Power and Energy Engineering Conference (APPEEC). IEEE, 2015, pp. 1-5.

[30] S. Gao, K. Chau, C. Chan, and D. Wu, "Loss analysis of vehicle-to-grid operation," in 2010 IEEE Vehicle Power and Propulsion Conference. IEEE, 2010, pp. 1-6.

[31] A. El-Zonkoly and L. dos Santos Coelho, "Optimal allocation, sizing of phev parking lots in distribution system," International Journal of Electrical Power Energy Systems, vol. 67, pp. 472-477, 2015.

[32] F. Shahnia, A. Ghosh, G. Ledwich, and F. Zare, "Voltage unbalance sensitivity analysis of plug-in electric vehicles in distribution networks," in AUPEC 2011. IEEE, 2011, pp. 1-6.

[33] M. Mazidi, A. Abbaspour, M. Fotuhi-Firuzabad, and M. Rastegar, "Optimal allocation of phev parking lots to minimize distribution system losses," in 2015 IEEE Eindhoven PowerTech, 06 2015, pp. 1-6. 
[34] P. Alluri, J. Solanki, and S. K. Solanki, "Charging coordination of plug-in electric vehicles based on the line flow limits and power losses," in 2015 International Conference on Technological Advancements in Power and Energy (TAP Energy), 06 2015, pp. 233-238.

[35] Z. Yang, X. Yu, and G. Holmes, "Evaluating impact of plug-in hybrid electric vehicle charging on power quality," in 2011 International Conference on Electrical Machines and Systems, 08 2011, pp. 1-4.

[36] M. R. Aghaebrahimi, M. M. Ghasemipour, and A. Sedghi, "Probabilistic optimal placement of ev parking considering different operation strategies," in MELECON 2014 - 2014 17th IEEE Mediterranean Electrotechnical Conference, 04 2014, pp. 108-114.

[37] M. R. Khalghani, S. Khushalani-Solanki, and J. Solanki, "Optimal integration and location of phev aggregators in power distribution systems," in 2016 North American Power Symposium (NAPS). IEEE, 2016, pp. 1-6.

[38] M. R. Khalghani, M. A. Shamsi-Nejad, and K. Beyki, "An intelligent controller for optimal vector control of induction motor," in 2011 IEEE International Conference on Computer Applications and Industrial Electronics (ICCAIE). IEEE, 2011, pp. 78-81.

[39] W. Kersting, Distribution System Modeling and Analysis, Third Edition. Taylor \& Francis, 2012. [Online]. Available: https://books.google.com/books?id=a-MY5JLUTKYC

[40] M. R. Khalghani, M. A. Shamsi-nejad, and M. H. Khooban, "Dynamic voltage restorer control using bi-objective optimisation to improve power quality's indices," IET Science, Measurement Technology, vol. 8, no. 4, pp. 203-213, 06 2014.

[41] S. D. Manshadi and M. E. Khodayar, "Resilient operation of multiple energy carrier microgrids," IEEE Transactions on Smart Grid, vol. 6, no. 5, pp. 22832292, 092015.

[42] A. Abaspour, N. T. Parsa, and M. Sadeghi, "A new feedback linearizationnsga-ii based control design for pem fuel cell," 2014.

[43] A. Abaspour, S. H. Sadati, and M. Sadeghi, "Nonlinear optimized adaptive trajectory control of helicopter," Control Theory and Technology, vol. 13, no. 4, pp. 297-310, 11 2015. [Online]. Available: https://doi.org/10.1007/s11768015-4062-1

[44] M. H. Khooban, T. Niknam, F. Blaabjerg, and T. Dragičević, "A new load frequency control strategy for micro-grids with considering electrical vehicles," Electric Power Systems Research, vol. 143, pp. 585 - 598, 2017. [Online]. Available: http://www.sciencedirect.com/science/article/pii/S0378779616304643 
[45] A. Khodabakhshian and R. Hooshmand, "A new pid controller design for automatic generation control of hydro power systems," International Journal of Electrical Power Energy Systems, vol. 32, no. 5, pp. 375 - 382, 2010. [Online]. Available: http://www.sciencedirect.com/science/article/pii/S0142061509001793

[46] R. Verma and S. P. and, "Intelligent automatic generation control of two-area hydrothermal power system using ann and fuzzy logic," in 2013 International Conference on Communication Systems and Network Technologies, 04 2013, pp. $552-556$.

[47] H. A. Yousef, K. AL-Kharusi, M. H. Albadi, and N. Hosseinzadeh, "Load frequency control of a multi-area power system: An adaptive fuzzy logic approach," IEEE Transactions on Power Systems, vol. 29, no. 4, pp. 1822-1830, 072014.

[48] R. K. Sahu, S. Panda, and N. K. Yegireddy, "A novel hybrid deps optimized fuzzy pi/pid controller for load frequency control of multi-area interconnected power systems," Journal of Process Control, vol. 24, no. 10, pp. 1596 - 1608, 2014. [Online]. Available: http://www.sciencedirect.com/science/article/pii/S0959152414002182

[49] M. Suman, M. Venu Gopala Rao, G. R. S. Naga Kumar, and O. Chandra Sekhar, "Load frequency control of three unit interconnected multimachine power system with pi and fuzzy controllers," in 2014 International Conference on Advances in Electrical Engineering (ICAEE), 01 2014, pp. 1-5.

[50] S. Firdous and M. A. R. Uzair, "Load frequency control techniques," 2015.

[51] M. H. Khooban and T. Niknam, "A new intelligent online fuzzy tuning approach for multi-area load frequency control: Self adaptive modified bat algorithm," International Journal of Electrical Power \& Energy Systems, vol. 71, pp. 254 - 261, 2015. [Online]. Available: http://www.sciencedirect.com/science/article/pii/S0142061515001520

[52] A. Kumar, A. Kumar, and S. Chanana, "Genetic fuzzy pid controller based on adaptive gain scheduling for load frequency control," in 2010 Joint International Conference on Power Electronics, Drives and Energy Systems 2010 Power India, 12 2010, pp. 1-8.

[53] D. K. and, "Fuzzy logic based load frequency control of grid connected distributed generators," in 2015 International Conference on Technological Advancements in Power and Energy (TAP Energy), 06 2015, pp. 432-437.

[54] S. S. Dhillon, J. S. Lather, and S. Marwaha, "Multi area load frequency control using particle swarm optimization and fuzzy rules," Procedia Computer Science, vol. 57, pp. 460 - 472, 2015, 3rd International Conference 
on Recent Trends in Computing 2015 (ICRTC-2015). [Online]. Available: http://www.sciencedirect.com/science/article/pii/S187705091501892X

[55] İlhan Kocaarslan and E. Çam, "Fuzzy logic controller in interconnected electrical power systems for load-frequency control," International Journal of Electrical Power 63 Energy Systems, vol. 27, no. 8, pp. 542 - 549, 2005. [Online]. Available: http://www.sciencedirect.com/science/article/pii/S0142061505000633

[56] E. B. M. Tayeb, "Automation of interconnected power system using fuzzy controller," in 2011 International Conference Utility Exhibition on Power and Energy Systems: Issues and Prospects for Asia (ICUE), 09 2011, pp. 1-5.

[57] C. Peng, J. Li, and M. Fei, "Resilient event-triggering $h_{\infty}$ load frequency control for multi-area power systems with energy-limited dos attacks," IEEE Transactions on Power Systems, vol. 32, no. 5, pp. 4110-4118, 092017.

[58] F. Daneshfar and H. Bevrani, "Multiobjective design of load frequency control using genetic algorithms," International Journal of Electrical Power Energy Systems, vol. 42, no. 1, pp. 257 - 263, 2012. [Online]. Available: http://www.sciencedirect.com/science/article/pii/S0142061512001287

[59] A. Sharifi, K. Sabahi, M. A. Shoorehdeli, M. A. Nekoui, and M. Teshnehlab, "Load frequency control in interconnected power system using multi-objective pid controller," in 2008 IEEE Conference on Soft Computing in Industrial Applications, 06 2008, pp. 217-221.

[60] I. Pan and S. Das, "Fractional-order load-frequency control of interconnected power systems using chaotic multi-objective optimization," Applied Soft Computing, vol. 29, pp. 328 - 344, 2015. [Online]. Available: http://www.sciencedirect.com/science/article/pii/S1568494615000137

[61] K. Naidu, H. Mokhlis, and A. Bakar, "Multiobjective optimization using weighted sum artificial bee colony algorithm for load frequency control," International Journal of Electrical Power \&3 Energy Systems, vol. 55, pp. 657 - 667, 2014. [Online]. Available: http://www.sciencedirect.com/science/article/pii/S0142061513004390

[62] K. S. Parmar, S. Majhi, and D. Kothari, "Load frequency control of a realistic power system with multi-source power generation," International Journal of Electrical Power Energy Systems, vol. 42, no. 1, pp. 426 - 433, 2012. [Online]. Available: http://www.sciencedirect.com/science/article/pii/S0142061512001676

[63] M. Rahmani and N. Sadati, "Two-level optimal load-frequency control for multi-area power systems," International Journal of Electrical Power 86 Energy Systems, vol. 53, pp. 540 - 547, 2013. [Online]. Available: http://www.sciencedirect.com/science/article/pii/S0142061513002123 
[64] C. Zhao, U. Topcu, and S. H. Low, "Optimal load control via frequency measurement and neighborhood area communication," IEEE Transactions on Power Systems, vol. 28, no. 4, pp. 3576-3587, 112013.

[65] C. E. Fosha and O. I. Elgerd, "The megawatt-frequency control problem: A new approach via optimal control theory," IEEE Transactions on Power Apparatus and Systems, vol. PAS-89, no. 4, pp. 563-577, 041970.

[66] M. Calovic, "Linear regulator design for a load and frequency control," IEEE Transactions on Power Apparatus and Systems, vol. PAS-91, no. 6, pp. 22712285, 111972.

[67] W. R. Barcelo, "Effect of power plant response on optimum load frequency control system design," IEEE Transactions on Power Apparatus and Systems, vol. PAS-92, no. 1, pp. 254-258, 011973.

[68] and and B. K. and, "Optimal tracking approach to load frequency control in power systems," in 2000 IEEE Power Engineering Society Winter Meeting. Conference Proceedings (Cat. No.00CH37077), vol. 2, 01 2000, pp. 1371-1376 vol.2.

[69] V. R. Moorthi and R. P. Aggarwal, "Suboptimal and near-optimal control of a load-frequency-control system," Proceedings of the Institution of Electrical Engineers, vol. 119, no. 11, pp. 1653-1660, 111972.

[70] — - "Damping effects of excitation control in load-frequency control system," Proceedings of the Institution of Electrical Engineers, vol. 121, no. 11, pp. 1409-1416, 111974.

[71] K. Sudha and R. V. Santhi, "Load frequency control of an interconnected reheat thermal system using type-2 fuzzy system including smes units," International Journal of Electrical Power Energy Systems, vol. 43, no. 1, pp. 1383 - 1392, 2012. [Online]. Available: http://www.sciencedirect.com/science/article/pii/S0142061512003286

[72] J. R. Castro and O. Castillo, "Interval type-2 fuzzy logic for intelligent control applications," in NAFIPS 2007 - 2007 Annual Meeting of the North American Fuzzy Information Processing Society, 06 2007, pp. 592-597.

[73] K. Sabahi, S. Ghaemi, and S. Pezeshki, "Application of type-2 fuzzy logic system for load frequency control using feedback error learning approaches," Applied Soft Computing, vol. 21, pp. 1 - 11, 2014. [Online]. Available: http://www.sciencedirect.com/science/article/pii/S1568494614000921

[74] M. R. Khalghani, M. H. Khooban, E. Mahboubi-Moghaddam, N. Vafamand, and M. Goodarzi, "A self-tuning load frequency control strategy for microgrids: Human brain emotional learning," International Journal of Electrical Power Energy Systems, vol. 75, pp. 311-319, 2016. 
[75] K. Vrdoljak, N. Peric, and M. Mehmedovic, "Optimal parameters for sliding mode based load-frequency control in power systems," in 2008 International Workshop on Variable Structure Systems, 06 2008, pp. 331-336.

[76] X. Meng, Q. Gong, L. Feng, W. Zheng, and W. Zhang, "Pi fuzzy sliding mode load frequency control of multiarea interconnected power systems," in Proceedings of the 2003 IEEE International Symposium on Intelligent Control. IEEE, 2003, pp. 1023-1027.

[77] T. A. Kumar and N. V. Ramana, "Design of optimal sliding mode controller for load frequency control in multi-area deregulated thermal system," in 2012 International Conference on Emerging Trends in Electrical Engineering and Energy Management (ICETEEEM), 12 2012, pp. 44-51.

[78] K. Vrdoljak, N. Perić, and I. Petrović, "Sliding mode based loadfrequency control in power systems," Electric Power Systems Research, vol. 80, no. 5, pp. 514 - 527, 2010. [Online]. Available: http://www.sciencedirect.com/science/article/pii/S0378779609002673

[79] B. D. Rittenhouse and A. Sinha, "Optimal sliding mode gaussian controller for a hydropower plant," in 2013 American Control Conference, 06 2013, pp. $6535-6540$.

[80] Y. Mi, Y. Yang, H. Zhang, A. Yu, L. Wang, and L. Ren, "Sliding mode based load frequency control for multi-area interconnected power system containing renewable energy," in 2014 IEEE Conference and Expo Transportation Electrification Asia-Pacific (ITEC Asia-Pacific). IEEE, 2014, pp. 1-6.

[81] Y. Mi, Y. Fu, D. Li, C. Wang, P. C. Loh, and P. Wang, "The sliding mode load frequency control for hybrid power system based on disturbance observer," International Journal of Electrical Power Energy Systems, vol. 74, pp. 446 - 452, 2016. [Online]. Available: http://www.sciencedirect.com/science/article/pii/S0142061515003002

[82] H. Kondo, Y. Suzuki, and S. Iwamoto, "A load frequency control design using the sliding mode control theory and disturbance observer," in 2010 Conference Proceedings IPEC, 10 2010, pp. 159-164.

[83] T. Anil Kumar and N. V. Ramana, "Notice of retraction lt;br gt; tuning of sliding mode observer optimal parameters for load frequency control in coordination with frequency controllable hvdc link in multi area deregulated power system," in 2015 International Conference on Electrical, Electronics, Signals, Communication and Optimization (EESCO), 01 2015, pp. 1-6.

[84] X. Guo and X. Liu, "Particle swarm optimization sliding mode control on interconnected power system," in Proceedings of the 33rd Chinese Control Conference, 07 2014, pp. 93-97. 
[85] A. Rahman, L. C. Saikia, and N. Sinha, "Load frequency control of a hydrothermal system under deregulated environment using biogeography-based optimised three-degree-of-freedom integral-derivative controller," IET Generation, Transmission Distribution, vol. 9, no. 15, pp. 2284-2293, 2015.

[86] M. Sathya and M. M. T. Ansari, "Design of biogeography optimization based dual mode gain scheduling of fractional order pi load frequency controllers for multi source interconnected power systems," International Journal of Electrical Power Energy Systems, vol. 83, pp. 364 - 381, 2016. [Online]. Available: http://www.sciencedirect.com/science/article/pii/S014206151630638X

[87] D. Guha, P. K. Roy, and S. Banerjee, "Load frequency control of interconnected power system using grey wolf optimization," Swarm and Evolutionary Computation, vol. 27, pp. 97 - 115, 2016. [Online]. Available: http://www.sciencedirect.com/science/article/pii/S2210650215000796

[88] N. Kumari and A. N. Jha, "Particle swarm optimization and gradient descent methods for optimization of pi controller for agc of multi-area thermalwind-hydro power plants," in 2013 UKSim 15th International Conference on Computer Modelling and Simulation, 04 2013, pp. 536-541.

[89] P. Dash, L. C. Saikia, and N. Sinha, "Agc of a multi-area interconnected system with facts and firefly optimized 2dof pid controller," in Proceedings of The 2014 International Conference on Control, Instrumentation, Energy and Communication (CIEC), 01 2014, pp. 397-401.

[90] N. E. Y. Kouba, M. Menaa, M. Hasni, B. Boussahoua, and M. Boudour, "Optimal load frequency control in interconnected power system using pid controller based on particle swarm optimization," in 2014 International Conference on Electrical Sciences and Technologies in Maghreb (CISTEM), 11 2014, pp. 1-8.

[91] Y. Tang, J. Yang, J. Yan, and H. He, "Intelligent load frequency controller using gradp for island smart grid with electric vehicles and renewable resources," Neurocomputing, vol. 170, pp. 406 - 416, 2015. [Online]. Available: http://www.sciencedirect.com/science/article/pii/S0925231215008644

[92] C. Mu, W. Liu, W. Xu, R. Islam et al., "Observer-based load frequency control for island microgrid with photovoltaic power," International Journal of Photoenergy, vol. 2017, 2017.

[93] H. Keshtkar, F. D. Mohammadi, J. Ghorbani, J. Solanki, and A. Feliachi, "Proposing an improved optimal lqr controller for frequency regulation of a smart microgrid in case of cyber intrusions," in 2014 IEEE 27th Canadian Conference on Electrical and Computer Engineering (CCECE), 05 2014, pp. $1-6$.

[94] L. Dong, Y. Tang, H. He, and C. Sun, "An event-triggered approach for load frequency control with supplementary adp," IEEE Transactions on Power Systems, vol. 32, no. 1, pp. 581-589, 012017. 
[95] S. Wen, X. Yu, Z. Zeng, and J. Wang, "Event-triggering load frequency control for multiarea power systems with communication delays," IEEE Transactions on Industrial Electronics, vol. 63, no. 2, pp. 1308-1317, 022016.

[96] C. Peng, J. Li, and M. Fei, "Load frequency control in isolated micro-grids with electrical vehicles based on multivariable generalized predictive theory," Energies, vol. 8, no. 3, pp. 2145-2164, 2015.

[97] M. R. Khalghani, S. Khushalani-Solanki, and J. Solanki, "A load frequency control for microgrid including stochastic elements based on hebb learning," in 2017 North American Power Symposium (NAPS), 09 2017, pp. 1-6.

[98] M. R. Khalghani, J. Solanki, S. Khushalani-Solanki, and A. Sargolzaei, "Stochastic load frequency control of microgrids including wind source based on identification method," in 2018 IEEE International Conference on Environment and Electrical Engineering and 2018 IEEE Industrial and Commercial Power Systems Europe, 2018, pp. 1-6.

[99] J. Yang, Z. Zeng, Y. Tang, J. Yan, H. He, and Y. Wu, "Load frequency control in isolated micro-grids with electrical vehicles based on multivariable generalized predictive theory," Energies, vol. 8, no. 3, pp. 2145-2164, 2015.

[100] S. Sridhar, A. Hahn, M. Govindarasu et al., "Cyber-physical system security for the electric power grid." Proceedings of the IEEE, vol. 100, no. 1, pp. 210-224, 2012.

[101] M. R. Khalghani, M. A. Shamsi-Nejad, M. Farshad, and M. H. Khooban, "Modifying power quality' s indices of load by presenting an adaptive method based on hebb learning algorithm for controlling dvr," 2014.

[102] J. Morén and C. Balkenius, "A computational model of emotional learning in the amygdala." MIT Press, 2000.

[103] M. R. Khalghani, S. Khushalani-Solanki, and J. Solanki, "Load frequency control in a microgrid including electric vehicle using neuroscience based controllers," in Microgrids: Design, Applications and Control, R. Allen and E. Jacobs, Eds. Nova Science Press, 2018, ch. 5, pp. 121-150.

[104] M. R. Khalghani and M. H. Khooban, "A novel self-tuning control method based on regulated bi-objective emotional learning controller's structure with tlbo algorithm to control dvr compensator," Applied Soft Computing, vol. 24, pp. 912 - 922, 2014. [Online]. Available: http://www.sciencedirect.com/science/article/pii/S1568494614004165

[105] www.winddata.com, accessed: 2014-10-10.

[106] S. Mousavian, M. Erol-Kantarci, L. Wu, and T. Ortmeyer, "A risk-based optimization model for electric vehicle infrastructure response to cyber attacks," IEEE Transactions on Smart Grid, vol. 9, no. 6, pp. 6160-6169, 112018. 
[107] Y. Zhang, S. Gjessing, H. Liu, H. Ning, L. T. Yang, and M. Guizani, "Securing vehicle-to-grid communications in the smart grid," IEEE Wireless Communications, vol. 20, no. 6, pp. 66-73, 122013.

[108] C. Peng, J. Li, and M. Fei, "Resilient event-triggering $h_{\infty}$ load frequency control for multi-area power systems with energy-limited dos attacks," IEEE Transactions on Power Systems, vol. 32, no. 5, pp. 4110-4118, 092017.

[109] A. Sargolzaei, K. K. Yen, M. Abdelghani, S. Sargolzaei, and B. Carbunar, "Resilient design of networked control systems under time delay switch attacks, application in smart grid," IEEE Access, vol. 5, pp. 15 901-15 912, 2017.

[110] A. Sargolzaei, K. K. Yen, and M. N. Abdelghani, "Preventing time-delay switch attack on load frequency control in distributed power systems," IEEE Transactions on Smart Grid, vol. 7, no. 2, pp. 1176-1185, 2016.

[111] S. Xu, Y. Qian, and R. Q. Hu, "On reliability of smart grid neighborhood area networks," IEEE Access, vol. 3, pp. 2352-2365, 2015.

[112] R. Tan, H. H. Nguyen, E. Y. Foo, D. K. Yau, Z. Kalbarczyk, R. K. Iyer, and H. B. Gooi, "Modeling and mitigating impact of false data injection attacks on automatic generation control." IEEE Trans. Information Forensics and Security, vol. 12, no. 7, pp. 1609-1624, 2017.

[113] G. Liang, S. R. Weller, J. Zhao, F. Luo, and Z. Y. Dong, "The 2015 ukraine blackout: Implications for false data injection attacks," IEEE Transactions on Power Systems, vol. 32, no. 4, pp. 3317-3318, 072017.

[114] Y. Chen, S. Huang, F. Liu, Z. Wang, and X. Sun, "Evaluation of reinforcement learning based false data injection attack to automatic voltage control," IEEE Transactions on Smart Grid, 2018.

[115] L. Yang, Y. Li, and Z. Li, "Improved-elm method for detecting false data attack in smart grid," International Journal of Electrical Power E Energy Systems, vol. 91, pp. 183 - 191, 2017. [Online]. Available: http://www.sciencedirect.com/science/article/pii/S0142061516324243

[116] D. Xue, X. Jing, and H. Liu, "Detection of false data injection attacks in smart grid utilizing elm-based ocon framework," IEEE Access, vol. 7, pp. $31762-31773,2019$.

[117] X. Yang, P. Zhao, X. Zhang, J. Lin, and W. Yu, "Toward a gaussian-mixture model-based detection scheme against data integrity attacks in the smart grid," IEEE Internet of Things Journal, vol. 4, no. 1, pp. 147-161, 022017.

[118] X. Wang, X. Luo, M. Zhang, and X. Guan, "Distributed detection and isolation of false data injection attacks in smart grids via nonlinear unknown input observers," International Journal of Electrical Power \& 
Energy Systems, vol. 110, pp. 208 - 222, 2019. [Online]. Available: http://www.sciencedirect.com/science/article/pii/S0142061518336202

[119] M. R. Khalghani, S. Khushalani-Solanki, J. Solanki, and A. Sargolzaei, "Stochastic load frequency control of microgrids including wind source based on identification method," in Environment and Electrical Engineering and 2018 IEEE Industrial and Commercial Power Systems Europe (EEEIC/IECPS Europe), 2018 IEEE International Conference on. IEEE, 2018, pp. 1-6.

[120] Y. Cui, L. Xu, M. Fei, and Y. Shen, "Observer based robust integral sliding mode load frequency control for wind power systems," Control Engineering Practice, vol. 65, pp. 1-10, 2017.

[121] Y. Cui, M. Fei, and D. Du, "Design of a robust observer-based memoryless h control for internet congestion," International Journal of Robust and Nonlinear Control, vol. 26, no. 8, pp. 1732-1747, 2016.

[122] A. Ashok, M. Govindarasu, and V. Ajjarapu, "Online detection of stealthy false data injection attacks in power system state estimation," IEEE Transactions on Smart Grid, vol. 9, no. 3, pp. 1636-1646, 052018.

[123] S. Prasad, S. Purwar, and N. Kishor, "Non-linear sliding mode based load frequency control for power systems using unknown-input-observer," in Control Conference (ICC), 2017 Indian. IEEE, 2017, pp. 233-239.

[124] A. F. Taha, J. Qi, J. Wang, and J. H. Panchal, "Risk mitigation for dynamic state estimation against cyber attacks and unknown inputs," IEEE Transactions on Smart Grid, 2016.

[125] A. F. Taha, A. Elmahdi, J. H. Panchal, and D. Sun, "Unknown input observer design and analysis for networked control systems," International Journal of Control, vol. 88, no. 5, pp. 920-934, 2015.

[126] A. Ameli, A. Hooshyar, E. El-Saadany, and A. Youssef, "Attack detection and identification for automatic generation control systems," IEEE Transactions on Power Systems, 2018.

[127] S. Sridhar and M. Govindarasu, "Model-based attack detection and mitigation for automatic generation control," IEEE Transactions on Smart Grid, vol. 5, no. 2, pp. 580-591, 2014.

[128] N. Pogaku, M. Prodanovic, and T. C. Green, "Modeling, analysis and testing of autonomous operation of an inverter-based microgrid," IEEE Transactions on power electronics, vol. 22, no. 2, pp. 613-625, 2007.

[129] A. D. Banadaki, F. D. Mohammadi, and A. Feliachi, "State space modeling of inverter based microgrids considering distributed secondary voltage control," in 2017 North American Power Symposium (NAPS). IEEE, 2017, pp. 1-6. 
[130] A. D. Banadaki, A. Feliachi, and V. K. Kulathumani, "Fully distributed secondary voltage control in inverter-based microgrids," in 2018 IEEE/PES Transmission and Distribution Conference and Exposition (TESD). IEEE, 2018, pp. 1-9.

[131] F. D. Mohammadi, "Modeling, simulation and decentralized control of islanded microgrids," Ph.D. dissertation, West Virginia University, 2017.

[132] F. D. Mohammadi, H. Keshtkar, A. D. Banadaki, and A. Feliachi, "A novel cooperative distributed secondary controller for vsi and pq inverters of ac microgrids," Heliyon, vol. 5, no. 6, p. e01823, 2019.

[133] M. Rasheduzzaman, J. A. Mueller, and J. W. Kimball, "Reduced-order smallsignal model of microgrid systems," IEEE Transactions on Sustainable Energy, vol. 6, no. 4, pp. 1292-1305, 2015.

[134] M. R. Khalghani, S. Khushalani-Solanki, J. Solanki, and A. Sargolzaei, "Cyber disruption detection in linear power systems," in 2017 North American Power Symposium (NAPS). IEEE, 2017, pp. 1-6.

[135] M. Hou and P. C. Muller, "Design of observers for linear systems with unknown inputs," IEEE Transactions on Automatic Control, vol. 37, no. 6, pp. 871-875, 061992.

[136] M. R. Khalghani, S. K. Solanki, J. Solanki, and A. Sargolzaei, "Resilient and stochastic load frequency control of microgrids," in 2019 IEEE Power 83 Energy Society General Meeting (PESGM). IEEE, 2019, pp. 1-5.

[137] A. Abbaspour, P. Aboutalebi, K. K. Yen, and A. Sargolzaei, "Neural adaptive observer-based sensor and actuator fault detection in nonlinear systems: Application in uav," ISA transactions, vol. 67, pp. 317-329, 2017.

[138] www.solargis.info/doc/solar-and-pv-data, accessed: 2014-10-10. 